NASA Contractor Report 3997

NASA-CR-3997 19860018614

\title{
Lateral Jet Injection Into Typical Combustor Flowfields
}

David G. Lilley

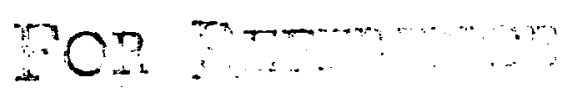

(n)

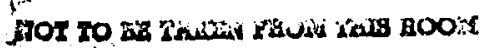

GRANT NAG3-549

JULY 1986

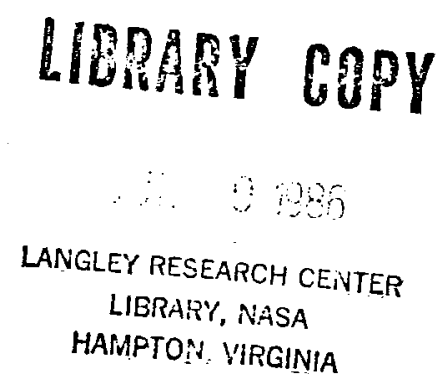


NASA Contractor Report 3997

\section{Lateral Jet Injection Into Typical Combustor Flowfields}

David G. Lilley

Oklahoma State University

Stillwater, Oklaboma

Prepared for

Lewis Research Center under Grant NAG3-549

\section{N/SA}

National Aeronautics

and Space Administration

Scientific and Technical Information Branch 
This Page Intentionally Left Blank 


\section{ABSTRACT}

This is the Final Report on Grant NAG 3-549 and discussion is on those activities undertaken during the entire one-year research program which began May 1, 1984 and ended April 30, 1985 with no-cost time extension to January 31, 1986. Studies were concerned with the experimental problem of lateral jet injection into typical combustor flowfields in the absence of combustion. All flowfields being investigated have no expansion of the crossflow (the test section to swirler diameter ratio $\mathrm{D} / \mathrm{d}=1$ ), after its passage through an optional swirler (with swirl vane angle $\phi=0$ (swirler removed), 45 and 70 degrees). The lateral jet(s) is(are) located one test-section diameter downstream of the test-section inlet $(x / D=1)$. The lateral jets have roundsectioned nozzles, each of which has an area of $1 / 100$ th of the cross-sectional area of the crossflow $\left(A_{j} / A_{c}=1 / 100\right)$. Jet-to-crossflow velocity ratios of $R$ $=v_{j} / u_{0}=2,4$, and 6 were investigated. Helium-bubble flow visualization, five-hole pitot probe time-mean velocity measurements, and single-wire timemean velocity and normal and shear stress turbulence data were obtained in the research program. 
This Page Intentionally Left Blank 


\section{CONTENTS}

ABSTRACT.............................................. ii

1. INTRODUCTION................................... 1

1.1 The Injection Problem.......................... 1

1.2 objectives...................................... 1

1.3 The Present Contribution........................ 2

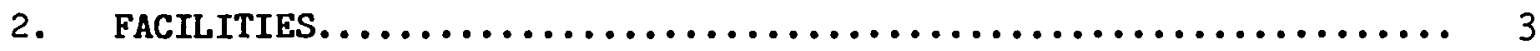

2.1 Wind Tunnel and Vane Swirler...................... 3

2.2 Test Section and Lateral Jet(s).................. 4

2.3 Pitot Probe Instrumentation..................... 5

2.4 Hot-Wire Instrumentation.......................... 6

2.5 Calibration Equipment.......................... 6

2.6 Data Acquisition and Probe Drive System.............. 7

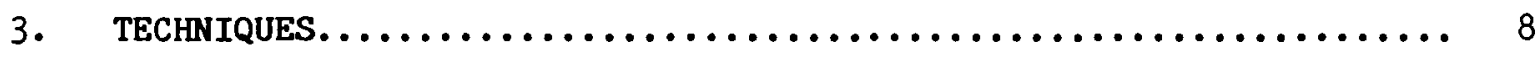

3.1 Bubble Flow Visualization....................... 8

3.2 Spark Flow Visualization......................... 9

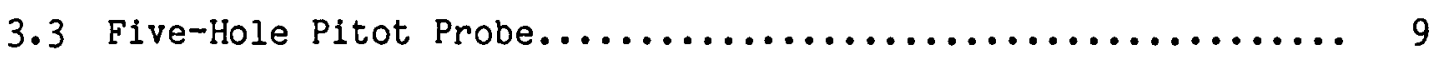

3.4 Multi-Orientation Single-Wire Hot-Wire............... 10

4. $\quad$ RESULTS..................................... 11

4.1 Predictive Studies............................ 11

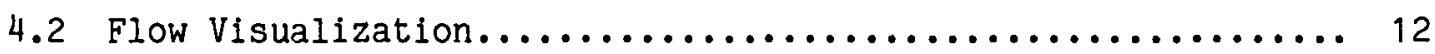

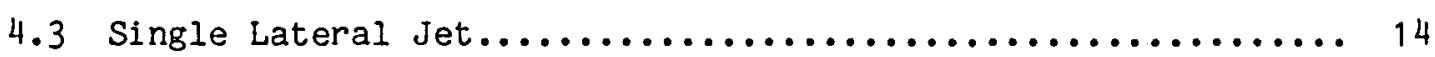

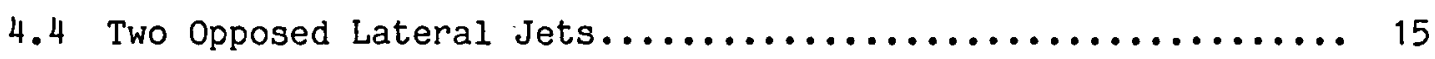

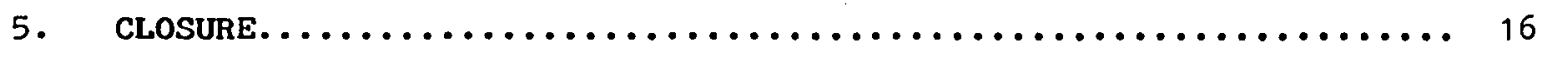

6. REFERENCES.................................. 17

6.1 References Supported by the Grant................. 17

6.2 Other References.............................. 18 
APPENDIXES

A. LATERAL JET INJECTION INTO TYPICAL COMBUSTOR FLOWFIELDS $\ldots \ldots \ldots \ldots 21$

B. FLOW VISUALIZATION OF LATERAL JET INJECTION INTO

SWIRLING CROSSFLOW.............................. 35

C. TURBULENCE MEASUREMENTS OF LATERAL JET INJECTION INTO

CONF INED TUBULAR CROSSFLOW........................ 47

D. HOT-WIRE MEASUREMENTS OF A SINGLE LATERAL JET INJECTED

INTO SWIRLING CROSSFLOW............................ 61

E. TWO OPPOSED LATERAL JETS INJECTED INTO SWIRLING CROSSFLOW....... 72

F. MULTISPARK FLOW VISUALIZATION OF LATERAL JET INJECTION

INTO A SWIRLING CROSS FLOW.......................... 87 


\section{INTRODUCTION}

\subsection{The Injection Problem}

In combustors of gas turbine engines, lateral injected jets are widely used to provide sufficient air for complete combustion as well as to evenly mix and cool the hot combustion products before their introduction to the turbine. As expected, the placement, size and injection velocities of lateral jets greatly affect the performance of the combustor in particular, and of the whole engine in general. Clearly, the flowfields in such situations are turbulent, reacting, and fully three-dimensional. Design and development of these combustors usually require the building of expensive prototypes followed by painstaking experiments, requiring lengthy and costly research programs. Time and expense could be greatly reduced with the availability of reliable computer codes for direct prediction of these complicated flowfields. The development of these, a subject of intensive present-day research, requires the existence of an accurate data base with which to compare predictions and further develop mixing models, finite difference schemes, and iterative procedures for improved accuracy and efficiency.

\subsection{Objectives}

The recently-completed research encompassed steady turbulent flow in a round cross-sectioned test section, with lateral jet(s) injected under low speed nonreacting conditions. All flowfields being investigated under this program have no expansion of the crossflow (the test section to swirler diameter ratio $\mathrm{D} / \mathrm{d}=1$ ), after its passage through an optional swirler (with swirl vane angle $\phi=0$ (swirler removed), 45 and 70 degrees). The lateral jet(s) is(are) located one test-section diameter downstream of the testsection inlet $(x / D=1)$. The lateral jets have round nozzles, each of which has an area of $1 / 100$ th of the cross-sectional area of the crossflow $\left(A_{j} / A_{c}=\right.$ 
1/100). Jet-to-crossflow velocity ratios $R=v_{j} / u_{0}=2,4$, and 6 , were studied in particular, and compared with previous no lateral jet injection data.

The principal aim was to investigate the trajectory, penetration and mixing efficiency of the lateral injection. Parameters investigated included:

1. lateral jet velocity (with velocity ratio $R=v_{j} / u_{0}=2,4$, and 6 ),

2. number of lateral jets (zero, one and two-opposed lateral jets),

3. crossflow swirl strength (with swirl vane angle $\phi=0$ (swirler removed), 45 and 70 degrees),

and their effects on the subsequent flowfield were observed and characterized.

\subsection{The Present Contribution}

The present document is the Final Report on Grant NAG 3-549 with discussion on these activities undertaken during the entire course of the 12month research project from May 1, 1984 to April 30, 1985 with no-cost time extension to January 31,1986 . One Ph.D. thesis ${ }^{1}$ and two M.S. theses 2,3 evolved in connection with the study, and these have been released as NASA contractor reports. In addition, five conference papers ${ }^{4-8}$ were prepared on various aspects of the work. The research program was largely experimental with a logical sequence of experiments being undertaken to establish the effects on the flowfield of lateral jet velocity, number of lateral jets, and crossflow swirl strength. Methods used included: flow visualization (using neutrally-buoyant helium-filled soap bubbles and multispark techniques), timemean velocity measurements (using a five-hole pitot probe) and turbulent normal and shear stress measurements (using a six-orientation single-wire hotwire technique).

Sections 2 and 3 describe facilities and techniques used in the 
investigation, while Section 4 highlights results obtained, with discussion on the coverage of relevant conference papers which are included as Appendixes A through F. The closure summarizes the study in Section 5 and a list of theses and papers appears in Section 6.

\section{FACILITIES}

\subsection{Wind Tunnel And Vane Swirler}

The test facility is described at length in the Appendixes. It consists of a wind tunnel, a variable-angle vane swirler, and a plexiglass test section of diameter $15 \mathrm{~cm}$. The wind tunnel has a axial-flow fan whose speed can be varied by altering a varidrive mechanism. Numerous fine screens and straws produce flow in the settling chamber of relatively low turbulence intensity. The contraction section leading to the test section has been specially designed to minimize adverse pressure gradient on the boundary layer and thus avoid unsteady problems associated with local separation regions. The airflow passes through a $15 \mathrm{~cm}$ diameter circular jet nozzle, exiting into a $15 \mathrm{~cm}$ diameter test section of length $90 \mathrm{~cm}$, which is constructed of plexiglass to facilitate flow visualization.

A variable-angle vane swirler may be positioned immediately before the test section. Its flow area has an outer diameter of $15 \mathrm{~cm}$. The swirler has ten vanes which are individually adjustable to any angle $\phi$ and a hub with a streamlined upstream nose and a flat downstream face. The nose has a hyperbolic shape with a very smooth surface so as to offer minimal flow interference. The flat blades are wedge-shaped to give a constant pitch-tochord ratio of 0.68 which gives good turning efficiency. Its performance was documented on the previous NASA research effort. ${ }^{9}$ The test section normally begins at a location $x / D=0$, which is $3.2 \mathrm{~cm}$ downstream of the swirler exit. Air to the lateral jet(s) is(are) supplied from a compressed air line via 
piping and carefully-designed nozzle(s). Upstream of each nozzle, a stagnation chamber, turbulence management screens, flow straightening straws, and flow metering equipment are used for flow conditioning. Experiments were performed with the lateral jet located one test-section diameter downstream of the inlet.

\subsection{Test Section and Lateral Jet(s)}

The test section is constructed from clear acrylic tubing. Of length 90 $\mathrm{cm}$, it is attached to the wind tunnel throat or immediately after the optional swirler. Standard commercial acrylic tube is used with $15.24 \mathrm{~cm}(6.0$ in.) outside diameter, $0.318 \mathrm{~cm}(0.125 \mathrm{in.})$ wall thickness. The inside diameter is $14.61 \mathrm{~cm}$ with a measured variation of $\pm 0.05 \mathrm{~cm}$. To adapt the test section to the wind tunnel throat (inside diameter $15 \mathrm{~cm}$ ), an adaptor section was machined to provide a smooth transition from wind tunnel throat to test section. Two test-section tubes were constructed. Both test sections have the dilution jet inlet located at $x / D=1.00$ where $x$ is measured from the tube inlet. The first tube has a series of probe access holes located at $x / D=$ $1.00,1.25$, and 1.50 and at all six azimuthal locations $270,300,330,0,30$, and 60 degrees. The second tube allows probe access to locations downstream of $x / D=1.50$ (for example $x / D=1.75,2.00,2.50,3.00$ ) and at any azimuthal angle. This is accomplished via a tube rotation section, constructed from machined aluminum rings, acrylic, and ball bearings.

The lateral jet assembly consists of a stagnation chamber, flow straightening section, and the jet nozzle. The stagnation chamber was constructed from $15 \mathrm{~cm}$ inside diameter aluminum pipe and filled with plastic household scrub pads to evenly distribute the internal flow. A hemispherically-shaped screen and convergent transition smooth the flow into the flow straightening section. Here the air flows through four brass screens 
for turbulence reduction. The nozzle is constructed of fiberglass with its precise contour being determined in a manner similar to that used for the main wind tunnel facility. The nozzle diameter one-tenth of the test-section diameter, giving a crossflow to jet area ratio $A_{c} / A_{j}=100$. Once assembled, the dilution jet was attached to the air line coming from the rotometer, and the nozzle was pressed into a special acrylic adaptor which is permanently attached to the test section.

The lateral jet(s) is(are) fed from laboratory compressed air at 6 to 7 atmospheres gauge pressure. For stability, the supply air lines are large and are routed through two line regulators with an intermediate tank (volume approximately $0.006 \mathrm{~m}^{3}$ ) to dampen line oscillations. The second regulator is used to meter the flow rate. After the second regulator, the air is routed through a Fisher and Porter model 10A1735A rotometer for monitoring of the volume flow before introduction to the lateral jet.

\subsection{Pitot Probe Instrumentation}

One of the simplest instruments capable of simultaneously sensing both magnitude and direction of the local velocity vector is the five-hole pitot probe, used extensively in the present and previous research studies. 9 . The particular probe employed was model DC-125-12-CD from United Sensor and Control Corp. It has a $3.2 \mathrm{~mm}$ diameter sensing tip and shaft containing five tubes. The sensing head is hook-shaped to allow probe shaft rotation without altering the probe tip location. The instrumentation system, in addition to the five-hole pitot probe, consists of a manual traverse mechanism, two fiveway ball valves, a very sensitive pressure transducer, a power supply, and an integrating voltmeter. The differential pressure transducer is model 590D from Datametrics, Inc. It has a differential pressure range of from 0 to $1.3 \times 10^{3} \mathrm{~N} / \mathrm{m}^{2}$ (equivalently, 0 to 10 torr). The integrating voltmeter 
is the TSI model 1076. As auxiliary equipment, a model 631-B strobotac from General Radio Inc, is used to check the fan speed. A pitot-static probe is used to measure the dynamic pressure in the nozzle throat just upstream of the swirler, and therefrom deduce the swirler inlet uniform axial velocity $u_{0}$ which is used later for velocity normalizations. Also, a barometer/ thermometer unit from Cenco Corp. is used for local pressure and temperature readings.

\subsection{Hot-Wire Instrumentation}

For the hot-wire measurements, the sensing transducer used in this study is a normal hot-wire probe, DISA type 55P01. This probe has two prongs set 3 $\mathrm{mm}$ apart with a $5 \mu \mathrm{m}$ diameter tungsten wire between them. The exposed, effective length of the wire is approximately $1 \mathrm{~mm}$, since the ends have been gold plated to strengthen the wire and reduce end effects. The probe support was a standard DISA $55 \mathrm{H} 21$ straight mounting tube. The anemometer used was a DISA type 55M01, constant-temperature standard bridge. The hot-wire voltage was measured with the computer-controlled data acquisition system, discussed in Section 2.6 .

\subsection{Calibration Equipment}

To calibrate the hot-wire, a small axisymmetric free jet was employed. The calibration jet facility consists of a contoured nozzle similar in shape to the dilution jet and wind tunnel contraction. A settling chamber and turbulence management section consisting of packed straws is just upstream of the nozzle. The nozzle exit diameter is $34 \mathrm{~mm}$. Using the standard laboratory air supply, the calibration jet is capable of producing Reynolds numbers up to $6 \times 10^{5}$ (based on throat diameter). The air supply is thermally stabilized by virtue of long indoor lines and is within $\pm 0.5^{\circ} \mathrm{C}$ of the facility temperature. The air is metered by means of a diaphragm valve and a Fisher 
and Porter model $10 \mathrm{~A} 1735 \mathrm{~A}$ rotometer. The jet was calibrated using a pitot probe located one nozzle diameter downstream of the exit plane, in the potential core region. The temperature of the jet and the pressure before the rotometer were monitored during each calibration to account for minor variations from the initial calibration velocities. The hot-wire was placed in the potential core of the jet during calibration. Utilizing a rotary table and two hot-wire support tubes (DISA 55H151 and DISA 55H153), the hot-wire was calibrated in the $u, v$ and $w$ directions.

\subsection{Data Acquisition and Probe Drive System}

The probe drive was specifically designed for these investigations. The probe is positioned in the flowfield by two stepper-motors, one motor for rotation and the other for translation. The probe is held within the square slider by a cylindrical holder with 0-rings to grasp the internal walls at any desired location. Both stepper motors step 200 times per motor shaft revolution. The rotation motor is geared down $3: 1$ so that 600 motor steps correspond to one probe revolution. For example, a probe rotation of 30 degrees requires exactly 50 steps. The software does not allow the probe to rotate in either direction more than one revolution to prevent cable twisting and coiling.

The translation motor is geared down $3: 1$ to a lead screw, which has a linear gear ratio of 2.24 revolutions per cm (equivalent to 5.69 revolutions per inch) translation. The effective step count for translation is therefore 1344 steps per $\mathrm{cm}$ (3414 steps per inch). With gear lash considered, the translation resolution is less than $0.03 \mathrm{~mm}$ and the rotation resolution is less than 0.5 degrees. The mass of the probe drive is approximately $3.9 \mathrm{~kg}$ (8.5 $\mathrm{Ibm})$ and it is fastened to the test section with a large rubber binding strap. 
An Apple II computer was used to sample the hot-wire voltage and control and the probe-drive stepper motors. A Burr-Brown SDM853 12-bit A/D converter was utilized to convert the $0-10$ volt hot-wire signals to 12-bit digital words. The Apple II controls, via assembly code, the sample times and accepts the data as two 8-bit words directly in RAM. Further machine codes are used to reassemble the samples, take an average and standard deviation and store the results. BASIC code is used to reassemble these 8 -bit words into decimal equivalent of 12-bit resolution. The system resolution is 2.44 millivolts. The data acquisition sample rate was fixed at 1000 samples per second for 5 seconds. A higher sampling rate (up to $30 \mathrm{kHz}$ ) could be utilized with more memory available.

\section{TECHNIQUES}

\subsection{Bubble Flow Visualization}

Flow visualization is used primarily for the identification and characterization of the flowfield, with two techniques being used in the present study: bubbles (described in the present section) and sparks (described in the next section).

Bubbles, because of their reflective qualities and neutral buoyancy in the airflow, provide an excellent medium to determine the paths of the jet trajectories. A bubble generator, manufactured by Sage Action, Inc. is used to supply up to 100 bubbles per second, and inject them into the lower lateral jet. The lighting for bubble flow visualization consists of light from a high-intensity slide projector located approximately $3 \mathrm{~m}$ downstream of the test section. A slit in an opaque slide provides a vertical light curtain about $1.5 \mathrm{~cm}$ wide. The lighting is on throughout the photography session and the exposure time of typically 5 seconds permits streamlines to be identified. The camera used is a Minolta SRI 200. The films used include 
Kodak Tri-X Pan 400 ASA black and white, Ilford 400 ASA black and white, and Kodak color 1000 ASA film, with all of these giving excellent results. The camera is positioned approximately $0.5 \mathrm{~m}$ laterally from the test section and supported by a tripod. A low F-stop of 2 is used for maximum light intake, to accentuate the bubble streaklines fllustrating the flow trajectories.

\subsection{Spark Flow Visualization}

A multi-spark visualization method has also been used on the present study. The technique uses an ionized path between two electrodes. This path moves with the air flow, and is sequentially lit up by successive sparks. The pulse generating circuit and pulse transformer are manufactured by Sugawara Laboratories, Inc., Tokyo.

When a high voltage source is sparked across an air gap, an ionized path is created. Subsequent sparks will follow the current position of this lowresistance ionized path. By placing electrodes in the wall boundary layer, where there is essentially zero velocity (next to the wall), several discharges can follow the ionized path as it moves with the fluid. The test section materials must have low electrical conductivity such as acrylic so as not to interfere with spark paths. The spark itself provides sufficient lighting for photographs. One camera (side view) is used for photographs with zero swirl. Two cameras (side and end view) are used simultaneously in the swirl crossflow cases to give added perspective to the three-dimensional features of the resulting flowfield.

\subsection{Five-Hole Pitot Probe}

Instrumentation for the five-hole pitot probe method for finding timemean velocity components in a general flowfield was described in Section 2.3. The probe is aligned with the flow yaw angle $\beta=\tan ^{-1}(w / u)$ in the plane perpendicular to the radius, while the pitch angle $\delta=\tan ^{-1}\left[v /\left(u^{2}+w^{2}\right)^{1 / 2}\right]$ 
and total velocity magnitude $V$ can then be found from previous corresponding free jet calibrations. The technique, associated computer code and user instructions are documented at length elsewhere. 10

The calibration of the five-hole pitot probe consists of two calibration characteristics: pitch angle $\delta$ versus differential pressure ratio $\left(P_{N}-\right.$ $\left.P_{S}\right) /\left(P_{C}-P_{W}\right)$, and velocity coefficient

$$
C=\rho V^{2} /\left[2\left(P_{C}-P_{W}\right)\right]
$$

versus pitch angle $\delta$.

With the measurement data of the differential pressure ratio $\left(\mathrm{P}_{\mathrm{N}}-\right.$ $\left.\mathrm{P}_{S}\right) /\left(\mathrm{P}_{\mathrm{C}}-\mathrm{P}_{\mathrm{W}}\right)$, the corresponding pitch angle $\delta$ is obtained with a cubic spline interpolation technique from the appropriate calibration characteristics. The velocity coefficient $C$ is determined from this value by using the corresponding calibration characteristics. The magnitude of the velocity vector is then calculated from

$$
V=\left[\frac{2}{\rho}\left(P_{C}-P_{W}\right) \cdot C\right)^{1 / 2}
$$

and the three time-mean velocity components are obtained from this value and the yaw and pitch angles.

\subsection{Multi-Orientation Single-Wire Hot-Wire}

Multi-orientation of a single hot-wire is a novel way to measure the three components of a velocity vector and their fluctuating components. In the present study, the six-orientation single-wire hot-wire method is used exclusively for detailed measurements. This method calls for a normal hot wire to be oriented through six different positions, each orientation separated by 30 degrees from the adjacent one. Orientation 1 is normal to the facility centerline, orientation 2 is rotated 30 degrees from this, etc. Time-mean and root-mean-square voltages are measured at each orientation. The data reduction is performed using several simplifying assumptions regarding 
the statistical nature of turbulence, making it possible to solve for three time-mean velocities, the three turbulent normal stresses, and the three turbulent shear stresses. ${ }^{11}$

The six-orientation hot-wire technique requires a single, straight, hot wire to be calibrated for three different flow directions in order to determine the directional sensitivity of the probe. When the wire is placed in a three-dimensional flowfield, the effective cooling velocity experienced by the hot wire may be deduced from the calibration curves. Hence, equations for the effective cooling velocity can be obtained for each of the six wire orientations. Simultaneously solving any three adjacent equations provides expressions for the instantaneous values of the three velocity components ( $u$, $v$, and $w$ in the facility $x, r$, and $\theta$ coordinates, respectively) in terms of the equivalent cooling velocities. It is then possible to obtain the three time-mean velocity components and the six different components of the Reynolds stress tensor, in the method described in Ref. 9. The accuracy and directional sensitivity of the technique were also assessed, concluding that inaccuracies may be present, especially in the deduced shear stress values. The configuration of probe versus local flow direction seems to be of little importance. Evaluating quantities from the average of all six possible wire combinations is recommended, and this smoothing has been used exclusively in recent studies and in the present study.

\section{RESULTS}

\subsection{Predictive Studies}

Theoretical computation of the fully 3-D flowfields occupied only a small part of the short research program. Preliminary results are given in Ref. 4 (see Appendix A), together with a review of jets in crossflow. Further details about the computational schemes used are given in Refs. 12 and 13 . 
This study emphasized the predictive capability for a single jet in crossflow, using comparisons with relevant flow visualization and pitot probe measurements available at that time. The fully 3-D code in cylindrical coordinates is based on the SOLA 2-D code from Los Alamos, using a uniform grid explicit forward-marching solution procedure. Velocity vectors in the $r x-p l a n e$ are given for different velocity ratios $R=0,2$ and 4 for the nonswirling and swirling crossflow cases of $\phi=0$ and 45 degrees. These tentative predictions are assessed in the light of smoke and bubble flow visualization pictures; the trends are generally predicted. It must be emphasized, however, that many more detailed computational studies have recently been undertaken elsewhere; the predictions of Appendix A are intended only to be demonstrative.

\subsection{Flow Visualization}

Extensive flow visualization experiments have been conducted to characterize the time-mean flowfield of a single and double (two opposed) deflected turbulent $j e t(s)$ in a confining cylindrical crossflow. They are reported in the research papers ${ }^{5,8}$ included as Appendixes $B$ (see also Appendix F) and $E$, respectively. Jet-to-crossflow velocity ratios of $R=2$, 4, and 6 were investigated, under crossflow inlet swirler vane angles of 0 (swirler removed), 45 and 70 degrees. Smoke, neutrally-buoyant helium-filled soap bubbles, and multi-spark flow visualization techniques were employed to highlight interesting features of the deflected jet, as well as the trajectory and spread pattern of the jet. Gross flowfield characterization was obtained for a range of lateral jet-to-crossflow velocity ratios and a range of inlet swirl strengths in the main flow. The flow visualization results are particularly useful for identifying regions of the flow in need of further detailed study. 
In Reference 5 (see Appendix B), smoke flow visualization combined with short time-duration photographs exhibited the organized eddy structure for low values of the jet-to-crossflow velocity ratio $R$. Values of $R=4$ and 6 exhibited large turbulent eddies. Both smoke and neutrally-buoyant heliumfilled soap bubbles were used to trace the jet penetration and expansion. The long time-exposure bubble photographs demonstrated the mean flow paths of the jet volumes. The case of $R=2$ was found to affect only the lower half of the test section. A multi-spark flow visualization technique was used to manifest changes in the flowfield after injection of the lateral jet. The technique demonstrated the bulk effects of the jet velocity ratio on the main flow, with a range of inlet swirl strengths in the main flow. Multi-spark photographs taken in the cases with swirl demonstrate the effect of lateral jet momentum on vortex core mixing and swirl strength, see Appendixes $\mathrm{B}$ and $\mathrm{F}$.

In Reference 8 (see Appendix E), flow visualization pictures (using bubbles and smoke) are given for the case of two opposed lateral jets entering swirling crossflow with $\phi=0,45$, and 70 degrees. The jets do not penetrate past the centerline of the test section in the nonswirling case, in contrast to the single lateral jet with the same jet-to-crossflow velocity ratio. In the swirling cases, the crossflow remains in a narrow region near the wall of the test section. The opposed jets are swept from their vertical courses into spiral trajectories close to the confining walls.

The flow visualization techniques enabled gross flowfield characterization to be obtained for a range of lateral jet-to-crossflow velocity ratios, a range of inlet swirl strengths in the main flow and the use of zero, one, and two lateral jets. The swirl in the confined crossflow was found to deflect the lateral jet(s) from its(their) vertical course in a spiral fashion. However, the jet still gets absorbed finally into the precessing 
vortex core of the crossflow. Evidence was also found that the jet can deflect the axis and hinder the upstream propagation of this vortex/core region toward the swirler.

\subsection{Single Lateral Jet}

Ferreli ${ }^{1,6}$ (see Appendix $C$ ) concentrated on the single lateral jet with various velocities entering nonswirling crossflow. He included smoke, bubbles and multispark flow visualization for the gross flowfield patterns, and the hot-wire technique for detailed time-mean and turbulence measurements in the $R$ $=2,4$, and 6 flowfields. Also described was how the six-orientation singlewire hot-wire technique was improved and could be used to measure the velocities and turbulent stresses. He applied the technique only to the nonswirling crossflow case, as a demonstration of improved data-acquisition capability. As described earlier, the computerized high-speed data acquisition and probe drive were designed and constructed to manipulate the hot-wire and reduce the varying voltages to the statistical mean and rootmean-square voltage. The voltages were then reduced to the time-mean velocity and turbulent Reynolds stresses with a Fortran computer code. The high-speed data acquisition system enabled three entire flowfields to be characterized for time-mean velocities, normal and shear stresses, for three different lateral jet injection velocities into nonswirling crossflow. The multiorientation technique worked well for time-mean velocities, normal stresses and most of the shear stresses. The extensive results are printed in tabular form and presented in rx-plane plots, sultable for subsequent use in flowfield modeling efforts. As expected, measurements confirmed that the deflected jet is symmetrical about the vertical plane passing through the crossflow axis. The jet penetration into the nonswirling crossflow was found to be reduced from that of comparable velocity ratio infinite crossflow cases, because of 
confinement effects.

Ong ${ }^{2,7}$ (see Appendix $D$ ) restricted the lateral jet velocity to conform to $R=4$ only, but added swirl to the crossflow with swirl vane angle $\phi=45$ and 70 degrees. The nonswirling case of $\phi=0$ degrees (swirler removed) had already been mapped in References 1 and 6 (see Appendix C). Experiments were conducted to document the time-mean and turbulent flowfield of a single deflected turbulent jet in a confined swirling crossflow. Again, the sixorientation single hot-wire technique was used to measure the velocities and turbulence properties of the flow. In addition, a five-hole pitot probe technique was used to measure the time-mean velocities for verification purposes. The results are presented in the form of $r-x$ plots to aid visualization of the fully three-dimensional flowfield. The swirl in the crossflow intensified the local velocity at the location of the injected jet, which effectively reduced the jet-to-crossflow velocity ratio. This caused the trajectory of the injected jet to follow the path of the local flow direction of the crossfiow, and reduce its penetration into the crossflow. The time-mean velocity measurements using the hot-wire corresponded to pitotprobe data obtained in identical flow conditions. Turbulence stress data show the same trends as previous swirl flow data without lateral injection. 9 The lateral jet was found to deflect the axis of the precessing vortex core.

\subsection{Two-Opposed Lateral Jets}

McMurry 3,8 (see Appendix E) experimented with two opposed lateral jets with $R=4$ entering swirling crossflow with $\phi=0$ (swirler removed), 45, and 70 degrees in the crossflow. This complemented the nonswirling single injection jet study of Ferrell 1,6 and the swirling single injection jet study of Ong, 2,6 see Appendixes $C$ and $D$ respectively. These experiments used the sixorientation single-wire hot-wire method to obtain the time-mean and turbulent 
quantities of opposed lateral jets in the low speed, nonreacting flowfield. The study included flow visualization where techniques used included neutrally-buoyant helium-filled soap bubbles and multi-spark photography in order to obtain the gross flowfield characteristics. Measurements of timemean and turbulent quantities were obtained utilizing the six-orientation single-wire hot-wire technique. For the nonswirling case, the jets were found not to penetrate past the test-section centerline, in contrast to the single lateral jet with the same jet-to-crossflow velocity ratio. In the swirling cases, the crossflow remains in a narrow region near the wall of the test section. The opposed jets are swept from their vertical courses into spiral trajectories close to the confining walls. Extensive results are presented in $r-x$ plane plots. The jets were also found not to deflect the axis of the precessing vortex core. In the case of moderate swirl, swirl vane angle $\phi=$ 45 degrees, the jets could be tracked following a spiral trajectory. With strong swirl, swirl vane angle $\phi=70$ degrees, the jet trajectories are difficult to determine. The data indicate that symmetry of the velocity profiles is disturbed because of the presence of the jets at the $x / D$ locations considered. Jet penetration into the crossflow is reduced due to the additional velocity component introduced into the crossflow by swirl.

\section{CLOSURE}

The main objectives of the research program were to determine the effects of the number of lateral jets, the jet velocity ratio, and the crossflow swirl strength on isothermal flowfield patterns in normally-injected jets into round cross-sectioned crossflow. The study included the investigation of the trajectory, penetration and mixing efficiency of the lateral injection. Experimental techniques included flow visualization, pitot probe and hot-wire methods. This final report concludes the present research on NASA Grant 
NAG 3-549, summarizing the activities, describing the facilities and techniques, and discussing major results obtained. Further details appear in the Ph.D. and M.S. theses that have evolved, and in the conference research papers that have been written and appended to the present document.

\section{REFERENCES}

\subsection{References Supported by the Grant}

The following list of theses and research papers covers research conducted with support of the grant.

1. Ferrell, G. B., "Deflected Jet Experiments in a Turbulent Combustor Flowfield." Ph.D. Thesis, Oklahoma State University, Stillwater, OKla., Dec. 1984. See also: Ferrell, G. B. and Lilley, D. G. "Deflected Jet Experiments in a Turbulent Combustor Flowfield." NASA CR-174863, May 1985 .

2. Ong, L. H., "Measurements of a Single Lateral Jet Injected into Swirling Crossflow." M.S. Thesis, Oklahoma State University, Stillwater, Okla., Dec. 1985. See also: Ong, L. H. and Lilley, D. G. "Measurements of a Single Lateral Jet Injected into Swirling Crossflow." NASA CR-175040, Jan. 1986 .

3. McMurry, C. B., "Experiments on Two Opposed Lateral Jets Injected into Swirling Crossflow." M.S. Thesis, Oklahoma State University, Stillwater, Okla., Dec. 1985. See also: McMurry, C. B. and Lilley, D. G., "Experiments on Two Opposed Lateral Jets Injected into Swirling Crossflow." NASA CR-175041, Jan. 1986. 
4. Ferrell, G. B., Abujelala, M. T., Busnaina, A. A., Lilley, D. G., "Lateral Jet Injection into Typical Combustor Flowfields." Paper AIAA-84-0374, Reno, Nevada, Jan. 9-12, 1984.

5. Ferrell, G. B., Aoki, K. and Lilley, D. G. "Flow Visualization of Lateral Jet Injection into Swirling Crossflow." Paper AIAA-85-0059, Reno, Nevada, Jan. 14-17, 1985. See also: "Multispark Flow Visualization of Lateral Jet Injection Into a Swirling Cross Flow." Journal of Propulsion and Power, Vol. 1, No. 6, Nov.-Dec. 1985, pp. 485-487.

6. Ferrell, G. B. and Lilley, D. G. "Turbulence Measurements of Lateral Jet Injection into Confined Tubular Crossflow." Paper AIAA-85-1102, Monterey, Calif., July 8-10, 1985.

7. Ong, L. H., McMurry, C. B. and Lilley, D. G. "Hot-Wire Measurements of a Single Lateral Jet Injected into Swirling Crossflow." Paper AIAA-860055, Reno, Nevada, Jan. 6-9, 1986.

8. McMurry, C. B., Ong, L. H. and Lilley, D. G. "Two Opposed Lateral Jets Injected into Swirling Crossflow." Draft Paper, Oklahoma State University, Jan. 1986.

\subsection{Other References}

9. Lilley, D. G. "Investigations of Flowfields Found in Typical Combustor Geometries." Final Report on Grant NAG 3-74, NASA CR-3869, Feb. 1985. 
10. Rhode, D. L., "Predictions and Measurements of Isothermal Flowfields in Axisymmetric Combustor Geometries." Ph.D. Thesis, Oklahoma State University, Stillwater, Okla., Dec. 1981. See also Rhode, D. L. and Lilley, D. G., "Predictions and Measurements of Isothermal Flowfields in Axisymmetric Combustor Geometries," NASA CR-174916, May 1985.

11. Jackson, T. W., "Turbulence Characteristics of Swirling Flowfields." Ph.D. Thesis, Oklahoma State University, Stillwater, Okla., Dec. 1983. See also Jackson, T. W. and Lilley, D. G., "Turbulence Characteristics of Swirling Flowfields," NASA CR-174918, May 1985.

12. Busnaina, A. A., "Transient Three-Dimensional Predictions of Turbulent Flows in Cylindrical and Cartesian Coordinate Systems." Ph.D. Thesis, Oklahoma State University, Stillwater, Okla., July 1983.

13. Abujelala, M.T., "Confined Turbulent Swirling Recirculating Flow Predictions," Ph.D. Thesis, Oklahoma State Univerisity, Stillwater, Okla., June 1984. See also Abujelala, M. T. and Lilley D. G., "Confined Turbulent Swirling Recirculating Flow Predictions," MASA CR174917 , May 1985. 
This Page Intentionally Left Blank 
APPENDIX A

LATERAL JET INJECTION INTO TYPICAL COMBUSTOR FLOWFIELDS

AIAA-84-0374 


\title{
LATERAL JET INJECTION INTO TYPICAL COMBUSTOR FLOWFIELDS
}

\author{
G. B. Ferrell,* M. T. Abujelala*, A. A. Busnaina,* and D. G. Lilley** \\ Oklahoma State University \\ Stillwater, Okla.
}

\section{Abstract}

A research program is underway which deals both experimentally and theoretically with the problem of primary and dilution lateral jet injection into typical combustor flowfields in the absence of combustion. Parameter variations to be systematically investigated include: lateral jet velocity, combustor cross-flow inlet swirl strength, and downstream contraction nozzle. The basic aim is to characterize the time-mean and turbulence flowfield with a variety of parameter settings, recommend appropriate turbulence model advances, and implement and exhibit results of flowfield predictions. The present study emphasizes the predictive capability of a fully three-dimensional computer code with $k-\varepsilon$ turbulence model, with preliminary experimental evaluation using smoke and helium bubble flow visualization, and five-hole pitot probe time-mean velocity measurements.

\section{Introduction}

\subsection{Combustor Flowfields}

High-intensity combustion takes place in gas turbine combustion chambers. 1 Whereas most fuel burning takes place in the primary zone, the secondary zone is where combustion is almost completed, and the dilution zone is where further temperature reduction and uniformity is achieved. In both can and annular combustors, lateral jets of cooler air through round holes penetrate the flowfield. Some of these jets amalgamate with the swirl-induced central recirculation region of the primary zone and provide sufficient additional air for stoichiometric conditions. Other lateral jets provide additional air to help complete combustion in the secondary one and to cool and evenly mix the products in the dilution zone in preparation for the introduction of the flow to the turbine. Similar problems arise in ramjet combustors. clearly the turbulent reacting flowfield is fully three-dimensional; the combustion designer has a formidable problem in aerothermochemistry, and the research and development task is to provide a route which leads to the accomplishment of design objectives more quickly and less expensively than current practjce permits. Recent conferences ${ }^{2}, 3$ and textbooks 4 -6 extensively review progress and problems in this area.

* Graduate Student, School of Mechanical and Aerospace Engineering Member AIAA

** Professor, School of Mechanical and Aerospace Engineering, Associate Fellow AIAA

\subsection{The Present Contribution}

The present paper addresses research that is restricted to steady nonreacting turbulent flow in axisymmetric geometries being disturbed by the introduction of one lateral jet. Figure 1 shows the test facility schematically. Both the crossflow and lateral jet initiate from carefullydesigned round-sectioned nozzles, designed by the method of Morel to produce minimum adverse pressure gradients on boundary layers and thus avoid unsteady problems associated with local separation regions. Exit flows have specific airflow properties - typically with uniform axial velocity profiles and low turbulence levels. The in-coming cross-flow may possess a swirl component of velocity via passage through a variable-angle swirl-vane pack ( $\phi$ from 0 to 70 deg.), exiting into a test section discussed more completely in Section 3. With the lateral jet located one testsection diameter downstream of the main flow inlet, the test section may possess a contraction nozzle at two test-section diameters downstream of the inlet. The objective of the present study is the characterization of flows of this type in terms of the effects of inlet swirl strength, inlet to test section expansion ratio, lateral jet velocity, and downstream contraction nozzle existence on the resulting flowfield in terms of its time-mean quantities. Injection problems of this type, into round cross-sectioned cross-flow, have received little attention - there is a definite need for work in this area. The present continuing study aims to characterize the timemean and turbulence flowfield, obtaining valuable sets of test data, recommend appropriate turbulence model advances, and implement and exhibit results of flowfield predictions. of course, the later advances may be readily and usefully assimilated later into more complex flowfield simulation schemes. In the present paper, only the nonexpanding flow is investigated, with only a limited amount of swirl effects. Later studies will also extend the range of parameter variables which affect the flowfield.

1.3 Combustor Research at Oklahoma State University

Recently, experimental and theoretical research is being undertaken on 2-D axisymmetric geometries under low speed, nonreacting, turbulent, swirling flow conditions. Experiments are being conducted in a version of the facility shown in Fig. 1 and described later in Section 3 . The flow enters the test section and proceeds into a larger chamber (the expansion $D / d=2,1.5$ or 1) via a sudden or gradual expansion (side-wall angle $\alpha=90$ and 45 degrees). A weak or strong nozzle may be positioned downstream to form a contraction exit to the test section. Inlet swirl vanes are adjustable to a variety of vane angles 
Table 1. Previous Investigations of Jets in Cross-Flow

\begin{tabular}{|c|c|c|c|c|c|c|c|c|}
\hline REF. & $\begin{array}{c}\text { JET } \\
\text { DIAMETER } \\
(m m)\end{array}$ & $\begin{array}{l}\text { INCIDENT } \\
\text { ANGLE }\end{array}$ & $\begin{array}{l}\text { JET } \\
\text { INLET } \\
\text { STYLE }\end{array}$ & $\begin{array}{l}\text { CROSS-FLOW } \\
\text { VELOCITY } \\
(\mathrm{m} / \mathrm{s})\end{array}$ & $\begin{array}{l}\text { VELOCITY } \\
\text { RATIO }\end{array}$ & GEOMETRY & MEASURED & TECHNIQUE \\
\hline 27. & $\begin{array}{r}6.35,9.5 \\
12.7,15.9\end{array}$ & 90 & orifice & -- & $2-7$ & open & $\begin{array}{l}\text { penetration } \\
\text { parameters }\end{array}$ & $\begin{array}{l}T / C \text { and pitot } \\
\text { rakes }\end{array}$ \\
\hline 28. & -- & 90 & $\begin{array}{l}\text { square, ellip- } \\
\text { tícal orifice }\end{array}$ & 46 & $2-8$ & open & $\begin{array}{l}\text { penetration } \\
\text { and mixing }\end{array}$ & $\begin{array}{l}T / C \text { and pitot } \\
\text { rakes }\end{array}$ \\
\hline 29. & $\begin{array}{r}6.35,9.5 \\
12.7,15.9\end{array}$ & $\begin{array}{l}90,60 \\
45,30\end{array}$ & pipe & $71.6,121.9$ & $2.9-5.7$ & open & $\begin{array}{l}\text { penetration } \\
\text { and mixing }\end{array}$ & $\begin{array}{l}T / C \text { and pitot } \\
\text { rakes }\end{array}$ \\
\hline 30. & $12.7,25.4$ & 90 & orifice & -- & $4,6,8$ & open & $\begin{array}{l}\text { total press., } \\
\text { flow direction }\end{array}$ & \\
\hline 31. & 9.5 & 90 & pipe & 1.5 & $4,6,8$ & open & $\begin{array}{l}\text { velocity, turb- } \\
\text { ulence intensity, } \\
\text { entrainment }\end{array}$ & $\begin{array}{l}\text { oriented hot- } \\
\text { wire }\end{array}$ \\
\hline 32. & $\begin{array}{l}6.35,9.5, \\
12.7\end{array}$ & 90 & orifice & $0.914,3.66$ & $5,15,25,35$ & open & $\begin{array}{l}\text { profiles and } \\
\text { penetration }\end{array}$ & photographs \\
\hline 33. & -- & 90 & pipe & -- & $0.55-2.20$ & $\begin{array}{l}\text { multiple } \\
\text { jets }\end{array}$ & $\begin{array}{l}\text { penetration } \\
\text { and mixing }\end{array}$ & $\begin{array}{l}T / C \text { and total } \\
\text { press. probe }\end{array}$ \\
\hline 34. & 6.35 & $\begin{array}{l}45,60,90 \\
05,120,135\end{array}$ & pipe & 1.58 & $4,6,8$ & open & $\begin{array}{l}\text { trajectories, } \\
\text { velocities }\end{array}$ & hot-wire \\
\hline
\end{tabular}

35. ANALYTICAL MODELING, NO EXPERIMENTS

\begin{tabular}{|c|c|c|c|c|c|c|c|c|}
\hline 36. & 6.35 & 90 & pipe/nozzle & $6-9$ & $2.8-8.5$ & $\begin{array}{l}\text { square holes } \\
\text { confined }\end{array}$ & $\begin{array}{l}\text { velocity and } \\
\text { temp. profiles }\end{array}$ & $\begin{array}{l}T / C \text { and hot- } \\
\text { wire }\end{array}$ \\
\hline 37. & $6.35-25.4$ & 90 & orifice & 15 & $1.67-5.67$ & 2-dimensional & $\begin{array}{l}\text { penetration, } \\
\text { mixing }\end{array}$ & $T / C$ rakes \\
\hline 38. & 40.0 & 90 & pipe & 3.4 & $\begin{array}{c}2.37,3.95 \\
6.36\end{array}$ & $\begin{array}{l}\text { multiple } \\
\text { jets }\end{array}$ & $\begin{array}{l}\text { velocity, temp. } \\
\text { similarity } \\
\text { profiles }\end{array}$ & hot-wire \\
\hline 39. & $\begin{array}{l}3.23,4.57 \\
6.30,9.32\end{array}$ & 90 & pipe & 6,15 & $2.4-12.4$ & $\begin{array}{l}\text { confined } \\
\text { cylin- } \\
\text { drical } \\
\text { channel }\end{array}$ & $\begin{array}{l}\text { penetration, } \\
\text { mixing }\end{array}$ & $\begin{array}{l}\text { marker } \\
\text { nephelonetry }\end{array}$ \\
\hline 40. & 101.6 & $\begin{array}{c}45,60,75 \\
90,105\end{array}$ & pipe & -- & 4,8 & open & $\begin{array}{l}\text { vortex } \\
\text { strength }\end{array}$ & $\begin{array}{l}\text { pitot } \\
\text { probes }\end{array}$ \\
\hline 41. & 25.4 & 90 & pipe & 12 & $1.15 ; 2.30$ & open & $\begin{array}{l}\text { detailed velocity } \\
\text { and turbulence } \\
\text { field }\end{array}$ & $\begin{array}{l}\text { LDA, X-wire, } \\
\text { helium trace }\end{array}$ \\
\hline
\end{tabular}

42.

ANALYTICAL MODELING, NO EXPERIMENTS 
with values of $\phi=0,38,45,60$ and 70 degrees being emphasized. The objective is to determine the effect of these parameters or isothermal flowfield patterns, time-mean velocities and turbulence quantities, and to establish an improved simulation in the form of a computer prediction code equipped with a suitable turbulence model. The goal of the on-going research is to perform experiments and complementary computations with the idea of doing the necessary type of research that will yield improved calculation capability. This involves performing experiments where time-mean turbulence quantities are measured, and taking input conditions and running a computer code for a variety of test cases so as to compare predictions against experiment. Hence the validity of turbulence model modifications can be assessed. In fact, they are also being deduced directly from the measured stresses and velocity gradients. Recent studies $8-15$ have resulted in several conference and journal research papers which haye appeared in connection with the studies $16-22$. Helium bubble flow visualization, 16-17 five-hole pitot probe time-mean velocity measurements, $17,18,20$ and one-wire and two-wire hot-wjre normal and shear stress turbulence datal4,21,22 are being obtained in the experimental program. Turbulence mpdeling deductions and flowfield predictions ${ }^{16,19}$ are being made via a 2-D axisymmetric technique, released as the STARPIC computer code. ${ }^{22}$ Related work includes the addition of complex chemistry with pollutant emissions to this code, 24 and the developement of a simple explicit fully 3-D turbulent flow computer code in cylindrical polar coordinates 25 . This latter code is being used in the present study.

\subsection{Previous Studies on Jets in Cross-Flow}

Recent textbooks review and present the progress which has been made concerning the injection of jets into cross-flow. Schetz concentrates on the physics and modeling of injection and mixing in turbulent flows; Lefebvre ${ }^{4}$ appreciates that the phenomena are of paramount importance in the combustion and dilution zones, and reveals recent progress in the light of combustor design requirements. Figures and empirical formulas are given which illuminate

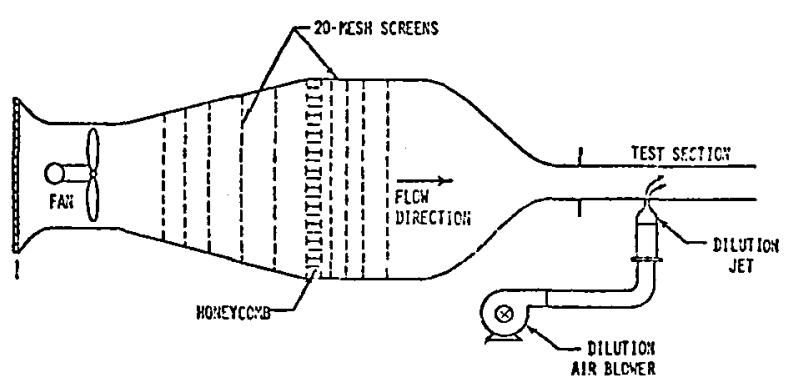

Fig. 1 The test facility.
Table 2. Schmidt Numbers and Source Terms in the General Equation.

\begin{tabular}{|c|c|c|}
\hline - & $\sigma_{0}$ & $s_{\phi}$ \\
\hline 1 & 0 & 0 \\
\hline u & 1 & $\begin{array}{l}\frac{w^{2}}{r}-\frac{\partial p}{\partial r}+\frac{1}{r} \frac{\partial}{\partial r}\left(r u_{\text {eff }} \frac{\partial u}{\partial r}\right)+\frac{1}{r}-\frac{\partial}{\partial \theta}\left(r r_{\text {eff }} \frac{\partial(w / r)}{\partial r}\right. \\
-2 \frac{\mu_{\text {eff }}}{r}\left[\frac{1}{r} \frac{\partial u}{\partial \theta}+\frac{v}{r}\right]+\frac{\partial}{\partial z}\left(u_{\text {eff }} \frac{\partial v}{\partial r}\right)\end{array}$ \\
\hline$\checkmark$ & 1 & $\begin{array}{l}-\frac{\partial p}{\partial z}+\frac{1}{r} \frac{\partial}{\partial r}\left(r u_{\text {eff }} \frac{\partial u}{\partial z}\right)+\frac{1}{r} \frac{\partial}{\partial \theta}\left(u_{\text {fff }} \frac{\partial w}{\partial z}\right) \\
+\frac{\partial}{\partial z}\left(u_{\text {eff }} \frac{\partial v}{\partial z}\right)\end{array}$ \\
\hline v & 1 & $\begin{array}{l}-\frac{w w}{r}-\frac{\partial p}{r \partial \theta}+\frac{1}{r} \frac{\partial}{\partial r}\left(r u_{e f f}\left[\frac{1}{r} \frac{\partial u}{\partial \theta}-\frac{w}{r}\right]\right) \\
+\frac{u_{e f f}}{r}\left[x \frac{\partial(w / r)}{\partial r}+\frac{1}{r} \frac{\partial u}{\partial \theta}\right] \\
+\frac{1}{r} \frac{\partial}{\partial \theta}\left(u_{e f f}\left[\frac{\partial u}{r \partial \theta}+\frac{2 u}{r} j\right)+\frac{\partial}{\partial z}\left(u_{e \in f} \frac{\partial v}{\partial \theta}\right)\right.\end{array}$ \\
\hline$m_{1}$ & 1 & 0 \\
\hline k & 1 & $G-C_{D}$ oE \\
\hline$\varepsilon$ & 1.3 & $\left(c_{1}{ } \varepsilon_{\varepsilon}-c_{2} \circ \varepsilon^{2}\right) / k$ \\
\hline
\end{tabular}

The rate of generation of $k$ by the action of velocity gradients is taken as:

$$
\begin{aligned}
c= & \mu_{\mathrm{r}}\left\{2\left(\frac{\partial v}{\partial z}\right)^{2}+2\left(\frac{\partial u}{\partial r}\right)^{2}+2\left(\frac{\partial w}{r \partial \theta}+\frac{u}{r}\right)^{2}\right. \\
& \left.+\left(\frac{\partial v}{\partial r}+\frac{\partial u}{\partial z}\right)^{2}+\left(\frac{\partial w}{\partial z}+\frac{2 v}{r \partial \theta}\right)^{2}+\left(\frac{\partial u}{r \partial \partial}+\frac{\partial w}{\partial r}-\frac{w}{r}\right)^{2}\right\}
\end{aligned}
$$

on how the rate of mixing between air jets and hot gases contained in the combustor liner is influenced by the following factors:

1. shape of the hole through which the jet issues,

2. initial angle of jet penetration,

3. velocity ratio,

4. density ratio,

5. length of the jet path,

6. turbulence properties of the jet,

7. presence of other jets, both adjacent and opposed,

8. proximity of walls,

9. inlet velocity profile of the jet and the hot gases.

Table 1 provides details from several papers $27-42$ on jets in cross-flow; these and other studies are reviewed elsewhere. 43 The table outlines parameters and techniques used by various investigators and the variables measured, including jet diameter, injection angle and style, and velocity ratio. Notice that injected flow through liner holes, orifices or pipes has inlet flow parameters which are not quantified well, and upon which mixing and flow penetration must depend. Typical of recent NASA-Lewis funded activity is the dilution jet mixing program 44 at Garrett Turbine Engine Co., whose main objective was to quantify by means of parametric tests the effect on the mixing of a row of jets with a confined cross-flow of: density ratio, flow area convergence, and nonuniformities in the cross-flgy further upstream. On the other hand, claus 45 performs analytic calculations of a single jet in cross-fiow and compares his results with appropriate experimental data. 


\subsection{Outline of the Paper}

In the present paper, a preliminary evaluation is made concerning the numerical computation of gross flowfield characteristics. The theoretical analysis of Section 2 discusses the prediction procedure. In Section 3, the facility and experimental details are given. Results are presented and discussed in Section 4, and the final closure summarizes the achievements.

\section{Computational Analysis}

\subsection{Governing Equations}

The turbulent Reynolds equations for conservation of mass, momentum, turbulent kinetic energy, and its dissipation rate are taken in 3-0 cylindrical polar coordinates in the common conservative form:

$$
\begin{aligned}
\frac{\partial \phi}{\partial t} & +\frac{1}{r} \frac{\partial}{\partial r}(\rho r u \phi)+\frac{1}{r} \frac{\partial}{\partial \theta}(\rho w \phi)+\frac{\partial}{\partial z}(\rho v \phi) \\
& =\frac{1}{r} \frac{\partial}{\partial r}\left(r \frac{\mu \text { eff }}{\sigma_{\phi}} \frac{\partial \phi}{\partial r}\right)+\frac{1}{r} \frac{\partial}{\partial \theta}\left(\frac{\mu \text { eff }}{\sigma_{\phi}} \frac{\partial \phi}{r \partial \phi}\right)+ \\
& \frac{\partial}{\partial x}\left(\frac{\mu \text { eff }}{\sigma_{\phi}} \frac{\partial \phi}{\partial z}\right)+S_{\phi}
\end{aligned}
$$

where $\phi$ stands for any of the dependent variables, $u, v, w, k$, and $\varepsilon$, and $\phi=1$ for the continuity equation. The time-mean velocity components $u, v$, and $w$ are in the $x, r$ and $\theta$ directions, respectively. The turbulent viscosity is calcylated from the standard $k$ $\varepsilon$ turbulence model: 25

$$
\begin{aligned}
& \mu_{t}=\rho C_{\mu} k^{2} / \varepsilon, \\
& \mu_{e f f}=\mu_{t}+\mu
\end{aligned}
$$

where $k$ is the turbulence energy and $\varepsilon\left[=k^{3 / 2} / \ell\right]$ is the energy dissipation rate, both of these being obtained from solution of their respective partial differential equations. The equations differ primarily in their Schmidt numbers $\sigma_{\text {, }}$ and final source terms $S$, as indicated $i n^{\phi}$ Table 2 . Turbulence and \&ther constants appearing in the tables are given the usual recommended values: $C_{D}$ $=1.0, C_{\mu}=0.09, C_{1}=1.44$, and $C_{2}=1.92$.

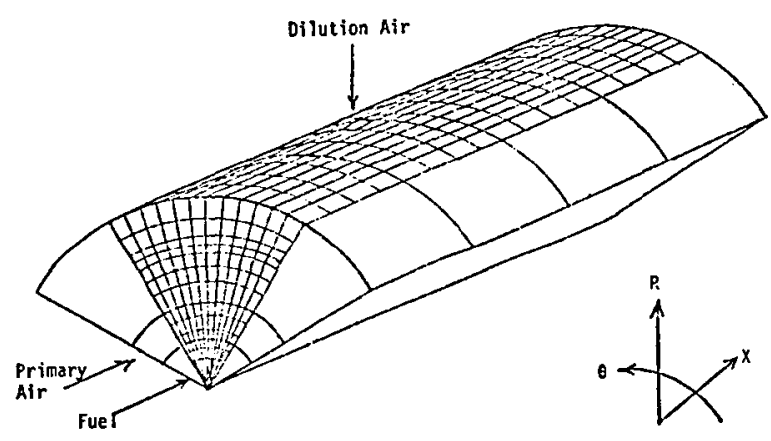

Fig. 2 Fully 3-D cylindrical grid schematic.
These values have been used in a wide variety of turbulent flow situations and exhibited good predictive capability. ${ }^{46}$

\subsection{Typical Grid System}

The three-dimensional region is divided into cell divisions with uniform spacings. The solution domain is complemented by a layer of cells on all sides, so as to allow easy simulation of the required boundary conditions. Figure 2 illustrates the total mesh arrangement for the cylindrical coordinate mesh arrangement. It represents the physical problem of a can combustor application, which involves a swirling jet penetration simulation in 60 degrees segment of a gas turbine combustor. Variables $p, k$, and $\varepsilon$ are cell-centered in each cell, whereas the three velocities are located normally on their respective cell faces. Thus normal velocities lie directly on the physical boundaries of the solution domain, while the tangential velocities and pressure are displaced half a cell interval inside the flowfield. In this way the exterior fictitious cells are particulary convenient when applying the boundary conditions.

\subsection{The Boundary Conditions}

Finite difference equations simulating the problem are set up and solved by way of a timemarch process applied to cells within the flow domain of interest. Cells touching the boundary thus utilize the value on the boundary (in the case of a normal velocity) or values half a cell distant beyond the boundary (in the case of tangential velocities).

Specifications of normal velocities at an outflow boundary often pose a problem, as it can have detrimental upstream influence. One might merely impose the zero-normal gradient or continuative condition and set these values equal to their immediate upstream values. When primary interest is being focused on the final steadystate solution, it has been found 25 that a suitable constant may be added to each such extrapolated value, with advantage to the rapidity of convergence. This constant value is chosen so as to make the total outlet flux equal to the total inlet flux, thus ensuring the requirement of a macroscopic mass balance.

Velocity specification at the inlets, symmetry axis and wall boundaries is in conformity with accepted turbulent flow calculation practice. Concerning turbulence quantities, the inlet value for the turbulent kinetic energy $k$ is taken as $0.03 u^{2}$ and the dissipation $\varepsilon$ is given by

$$
\varepsilon=k^{3 / 2} / \ell
$$

where $\ell$ is the length scale of turbulence, taken typically as 3 percent of the characteristic size of the passage. At a plane of symmetry both $k$ and $\varepsilon$ are given the zero-gradient condition. At a wall, $k$ is treated similarly but $\varepsilon$ values are specified just inside the computational field at a point $P$ in terms of the local value of $k$ via

$$
\varepsilon_{p}=\frac{c_{\mu}^{3 / 4} k_{p}{ }^{3 / 2}}{k \cdot y_{p}}
$$


Wall shear stress is evaluated via the modified log-law with the assumption of a constant shear region near the wall. Wall functions are used to avoid the need for detailed calculations near the wall. The one-dimensional Couette flow characterization (diffusion perpendicular to the wall is dominant) is used to link velocities, $k$ and $\varepsilon$ on the wall to those in the logarithmic region. The wall functions are used in the momentum equations and $k$-generation terms of nearwall points.

\subsection{The Solution Procedure}

Computations for one calculation cycle (timestep) can be summarized in three steps:

1. Computing the velocity at all internal points via application of momentum principles.

2. Adjusting the cell pressure and velocities iteratively to satisfy the continuity equation.

3. When convergence is achieved, the pressure and velocity values will be at the advanced time-level and can be used to start the calculation of the next time-step.

The progedure is based on the SOLA computer program. 47 Finite difference representations are required of the governing partial differential equations. The usual intuitive estimates of onesided first-derivatives, centered firstderivatives and centered second-derivatives are used in representing the momentum equations. Superscripts $n$ and (blank) are used to denote values at time-level $t$ and $t+\Delta t$, respectively. Portrayed now are equations enabling one such forward time-step to be accomplished. Thus, starting from initial field values throughout the domain of interest, a time-march process is used so as to advance toward the final steady-state solution, which is of special interest in the present case as opposed to the en route calculations. Full details about the finite difference equations, the transient forwardmarching solution scheme, iterative procedure, convergence and stapility are available in a recent $\mathrm{Ph} . \mathrm{D}$. Thesis. 25

\section{Experimental Approach}

\subsection{Facilities}

The following facilities are being used on the investigation: wind tunnel, one variableangle vane swirler, three plexiglass test sections of diameters $30,22.5$ and $15 \mathrm{~cm}$, expansion biocks of 90 and 45 deg., for each of the three test sections, and weak and strong downstream contraction nozzles, for each of the three test sections.

The experiments are being conducted in a modified version of the confined jet test facility which is described at length elsewhere. ${ }^{8-10}$ The facility has an axial-flow fan whose speed can be changed by altering a varidrive mechanism. Numerous fine screens and straws produce flow in the settling chamber of relatively low turbulence intensity. The contraction section leading to the test section has been designed by the method of Morel ${ }^{\gamma}$ to produce a minimum adverse pressure gradient on the boundary layer and thus avoid unsteady problems associated with local separation regions. The air-flow passes through a $15 \mathrm{~cm}$ diameter circular jet nozzle, exiting into a $15 \mathrm{~cm}$ diameter test section of length $90 \mathrm{~cm}$, which is constructed of plexiglass to facilitate flow visualization. A variable-angle vane swirler may be positioned immediately before the test section.

The swirler has ten vanes which are individually adjustable to any angle, $\phi$, and a hub with a streamlined upstream nose and a flat downstream face. The nose has a hyperbolic shape with a very smooth surface so as to offer minimal flow interference. The flat blades are wedgeshaped to give a constant pitch-to-chord ratio of 0.68 which should give good turning efficjency. Its performance is documented elsewhere. 13,20 The test section normally begins at a location $\times / D=$ 0 , which is $3.2 \mathrm{~cm}$ downstream of the swirler exit.

The effects of a downstream contraction nozzle on the upstream flow in the test section is important in combustor aerodynamics. In the present study, only the strong contraction nozzle of area ratio 4 is being used. Its upstream face is a 45 degree slope, typical of gas turbine combustion chamber exits. Its description and its effect on the upstream flow is discussed in Refs. $10-12,15,18,19$ and 22 . When used, it is typically located at two test-section diameters downstream of the inlet to the flow domain.

Figure 1 shows the initial configuration of the $15 \mathrm{~cm}$ diameter test section (the same as the exit throat) mounted on the wind tunnel with the swirler omitted. Air to the lateral jet is supplied from a compressed air line via piping and a carefully-designed nozzle. Upstream of each nozzle, a stagnation chamber, turbulence management screens, flow straightening straws, and flow metering equipment are used for flow conditioning. Experiments are being performed with the lateral jet located one test-section diameter downstream of the inlet. To allow probe access to investigate the nonaxisymmetric flow in the test section, a set of holes in the axial direction only is needed together with azimuthal rotation capability of the test section relative to the lateral inlet location.

\subsection{Measurement Technique}

Standard techniques of flow visualization are used, including the use of smoke injection and neutrally-bugyant helium-filled soap bubbles. 16,19 General flow patterns obtained in this way serve to identify regions of special interest and indicate locations in need of further detailed investigation. Time-mean velocities are being measured with a five-hole pitot probe, which has been successfully used in related studies.

It is a useful instrument when the need arizes to measure both the magnitude and direction of the velocity simultaneously in an unknown flowfield. The five-hole pitot probe used in this study is a model DC-125-12-CD manufactured by United Sensor and Control Corp. The sensing head is hook-shaped to allow for probe shaft rotation without altering the probe tip location. The instrumentation assembly, in addition to the five-hole pitot probe, is composed of a manual traverse mechanism, two five-way ball valves, a differential pressure transducer, a power supply and an integrating 
digital voltmeter. The differential pressure transducer is model 5900 from Datametrics, Inc. It has a differential pressure range of from 0 to $1.3 \times 10^{3} \mathrm{~N} / \mathrm{m}^{2}$. The integrating voltmeter is the TSI model 1076. As auxiliary equipment, a model 631-B strobotac from General Radio Inc. is used to check the fan speed. A micro-manometer, along with a pitot-static probe, is used to measure the dynamic pressure in the nozzle throat just upstream of the swirler, and therefrom deduce the swirler inlet uniform axial velocity $u_{0}$ which is used later for velocity normalizations. Also, a barometer/ thermometer unit from Cenco Corp. is used for local pressure and temperature readings.

\section{Results}

\subsection{Deflected Turbulent Jet}

An interesting three-dimensional problem arises when a turbulent jet enters normally into a uniform steady cross-flow. Figure 3 shows a schematic of the problem, which has previously

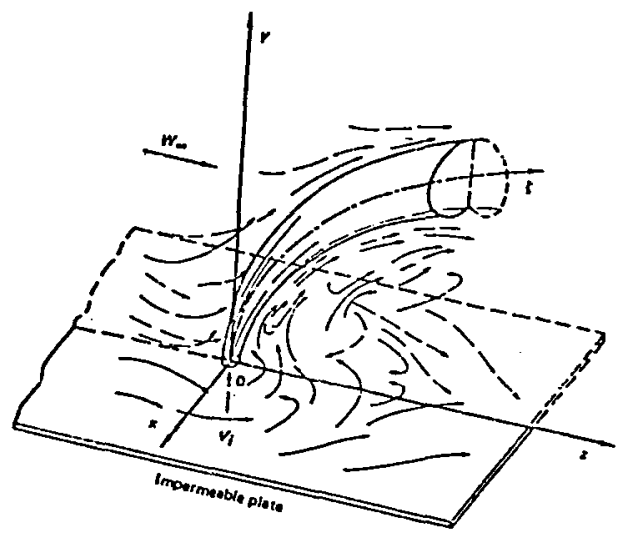

Fig. 3 Schematis of the deflected jet problem ${ }^{4}$.

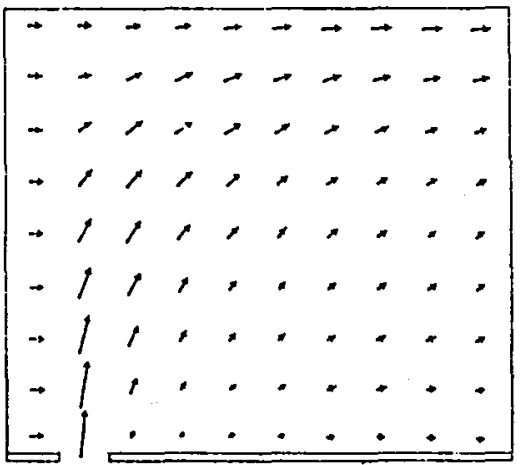

Fig. 4 Velocity vectors in downstream plane through lateral jet with velocity ratio $\mathrm{R}=6$. been discusseg, 48 predjcted 49 and experimentally investigated. $30,31,38,50$ Such problem configurations arise in chimney plumes, flow under a V/STOL aircraft, film cooling and dilution air mixing in combustor applications. Earlier predictions 49 utilized a sophisticated proprietary 3-D implicit computer code to obtain steady state predictions. For the present predictions, a Cartesian version of the code described in Section 2 is used. Use is made of the vertical symmetry plane and a horizontal top plate is located 18 jet radii above the bottom plane. A grid system of 9 $x 9 \times 7$ internal cells (in $x, y$, and $z$ directions, respectively) is used. Uniform steady crossflow is specified at the upstream plane of the main inflow to the domain with velocity $u_{0}$. The usual zero normal gradients are taken at the exit plane. Free slip boundary conditions are used on the top, bottom, and side planes.

Figure 4 shows the predicted velocity vectors in the $y z-p l a n e$ through $x=0$. This gives a clear indication of the behavior, magnitude, and direction of the deflected jet. These predictions are for the jet to cross-fiow velocity ratio $R=$ $v_{j} / u_{0}=6$, and the standard values in the $k-\varepsilon$ turbulence model are used. ${ }^{46}$ Velocity vector plots of this type allow the jet trajectory (the line joining points of maximum velocity) to be determined.

Experimental work for comparison purposes include: Jordinson, 30, Keffer and Baines (who studied the structure of turbulence), Ramsey and Goldstein, 50 (who presented velocity and temperature plots in cross-seffional and symmetry planes), and Chassaing et al ${ }^{38}$ (who studied both cylindrical and co-axial jets). The predicted effect of jet to cross-flow velocity ratio $R$ on jet trajectories is illustrated in Fig. 5 with appropriate experimental data for comparison.

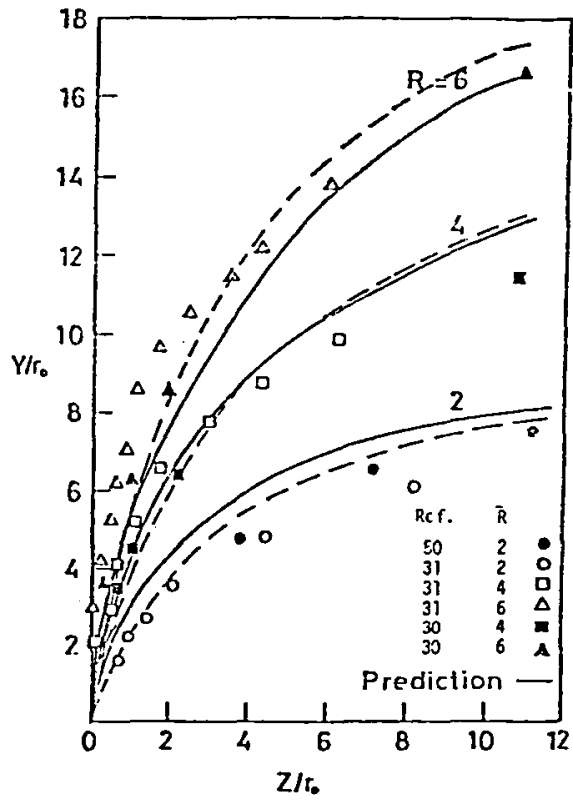

Fig. 5 Jet centerline locations for different velocity ratios [--- predictions ${ }^{49}$ ]. 
Earlier predictions ${ }^{49}$ are very much in agreement with the present study as shown in the figure. Effect of grid size on the solution is discussed and exemplified in Ref. 25. Predictions with a refined grid $(20 \times 20 \times 9)$ show better agreement with the experimental data than those with the coarser $(11 \times 11 \times 9)$ grid. The difference between the two predictions, however, is relatively small (7 percent) and considering the high cost of using a large grid size (about 23 minutes of computer time compared to 8 minutes for small grid size) the smaller grid size may be considered as a more practical choice. Considering the scatter in the data, the quality of the predictions is very good, thus confirming that the main dynamic and turbulence mixing effects are modeled adequately in the present code.

Comparison of predictions with experimental data and earlier predictions shows that this 3-0 simulation is useful for the prediction of the jet centerline location and velocity vectors. Predictions show, as expected, that the location of jet centerline is a strong function of the jetto-cross-flow velocity ratio.

\subsection{Dilution Jets in Gas Turbine Combustors}

The problem of dilution jets in gas turbine combustors is a design area which receives considerable attention during the design and development of combustors. Lateral jets are used to maintain a stable burning zone, dilute nearstoichiometric mixtures and to cool and evenly mix the products before introduction of the flow to the turbine. Computational models which provide 3-D predictions of velocities, pressure, temperatures, species, and concentrations are relatively new. Examples of application of these types of models have been presented in the literature. $51-54$

Computations. The present computations are for a 60 deg. sector of the flow-domain round cross-section, thus simulating a situation in which six lateral jets are located around the domain. A 3-D grid system in cylindrical coordinates as illustrated in Fig. 2 is used, with the dilution jet located one diameter downstream of the main inlet. A finite difference grid of size $10 \times 10 \times 7$ internal cells in the $x, r$ and $\theta$ directions is used. The $\theta$-boundary conditions of the solution domain are cyclic ones (periodic). That is, the given boundary condition will be that all the $\theta$-planes separated by multiples of $60 \mathrm{deg}$. are corresponding planes with identical profiles. Thus the $\theta$-boundary conditions are handled in a manner that preserves the cyclic status by letting the profile and gradient of the first $\theta$-plane equal the profile and gradient of the last $\theta$-plane. This periodic boundary condition applies not only to velocities and pressure, but also to all other dependent variables.

Predictions were made using inlet velocity profiles based on mean flowfield measurements for the axial velocity $u_{0}$ and tangential velocity $w_{0}$, with radial velocity $v_{0}$, given the value of zero at the inlet. Predicted time-mean velocities are shown for two cases:
1. Nonswirling flow with and without a dilution jet (shown in Fig. 6).

2. Swirling flow with and without a dilution jet in a low Reynolds number flow (shown in Fig. 7).

In the first case, shown in Fig. 6(a), the velocity vectors are showing magnitude and direction in the rx-plane for a nonswirling flow without lateral jet. Figure 6(b) illustrates the effect of the dilution jet with a jet-to-inlet velocity ratio of $R=v_{j} / u_{0}=2$, and Part (c) is with $R=4$. In these cases, the $r x-p l a n e$ shown is directly through the lateral jet. The predicted velocity vectors show the penetration and deflection of the dilution jet in the flow region. The situation may be compared with the jet to cross-flow ratio $R$ of 4 , as shown in Fig. 5 for Cartesian coordinates. It is clear that as the velocity of the lateral jet increases its penetration towards the axis increases, as expected.

In the second case, velocity vectors for a swirling flow with swirl vane angle $\phi=45$ deg. and zero dilution jet velocity are shown in Fig. 7 (a). The corresponding flow with a dilution jet that has a jet-to-inlet velocity ratios of 2 and 4 are shown in Parts (b) and (c) of the figure. The effect of swirl on the dilution jet penetration is shown in the latter vector velocity plots where the jet is seen to penetrate less than in the zero swirl case. This is because the injected air is given a swirl velocity component as it penetrates the flow domain, and its main zone is thus taken

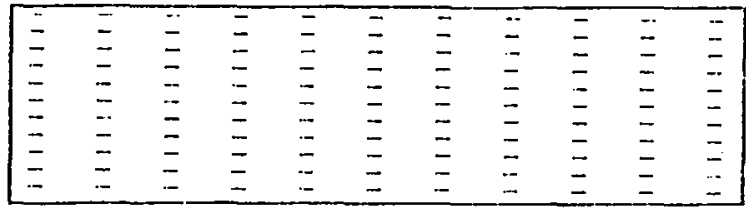

(a)

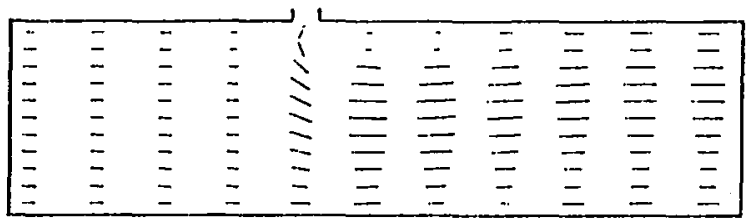

(b)

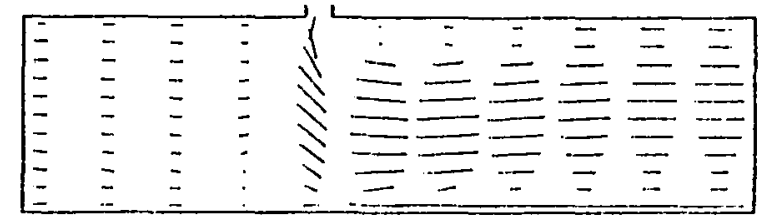

(c)

Fig. 6 Velocity vectors showing magnitude and direction in $r x-p l a n e$ through lateral jet with nonswirling main flow and velocity ratio $R=v_{j} / u_{0}$ of: (a) 0 (b) 2 (c) 4 . 


\begin{tabular}{|c|c|c|c|c|c|c|c|c|c|c|}
\hline$\therefore$ & $\because$ & $=$ & - & - & $=$ & - & - & - & - & - \\
\hline- & - & $=$ & $=$ & $=$ & $=$ & $\ddot{z}$ & $\Xi$ & $\bar{I}$ & $\vec{a}$ & $I$ \\
\hline$=$ & $\because$ & $=$ & $=$ & $=$ & - & - & - & - & - & - \\
\hline $\overrightarrow{-}$ & $=$ & $\bar{z}$ & $\bar{z}$ & $\overline{-}$ & $=$ & $=$ & $\ddot{-}$ & $=$ & $\Xi$ & $\approx$ \\
\hline $\overrightarrow{.}$ & $=$ & $\vec{z}$ & 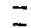 & $=$ & $\bar{z}$ & $=$ & $=$ & $\bar{z}$ & $\bar{z}$ & $\vec{z}$ \\
\hline- & - & - & - & - & - & - & 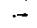 & - & - & $=$ \\
\hline- & $\therefore$ & - & $=$ & $\Xi$ & $=$ & \pm & $=$ & 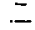 & $=$ & - \\
\hline
\end{tabular}

(a)

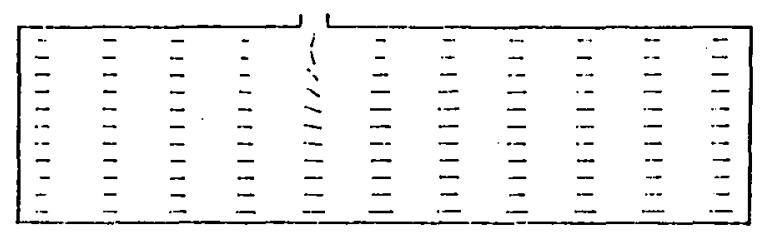

(b)

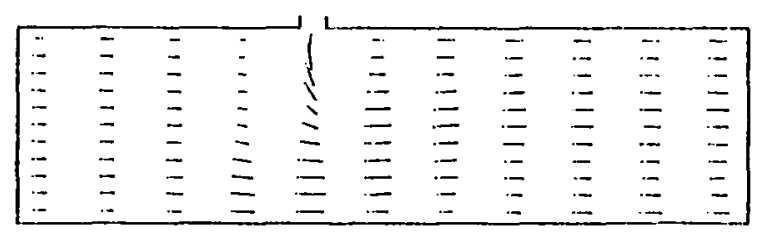

(c)

Fig. 7 Velocity vectors showing magnitude and direction in rx-plane through lateral jet with swirling main flow ( $\phi=45$ deg) and velocity ratio $R=v_{j} / u_{0}$ of: (a) 0 (b) 2 (c) 4

out of the rx-plane shown, which is through the lateral jet inlet. It therefore has more capability to spread its effect than was the case in Fig. 6, in which no swirl was present.

Dilution jets in gas turbine combustors constitute a fuily 3-0 problem which must be simulated using cylindrical coordinates system. The developed prediction procedure represents a basic tool to show the influence of design parameters on the dilution zone in combustors. Predicted results show the expected trends in the flow such as the dilution jet deflection. Comparison with earlier predictions shows that the quality of the predicted results is very good, thus confirming that the main dynamic and the turbulence mixing effects are modeled adequately.

Flow Visualization. Figure 8 shows very short time exposures of smoke tracing the extent of the lateral jet with jet to cross-flow velocity ratio $R$ of $2,3,4$ and 6 in Parts (a) through (d), respectively The exposure time is of the order of $1 \times 10^{-4} \mathrm{~s}$, and vertical slit lighting is obtained with two commercial flash units. Notice that as the lateral jet velocity increases, so also does the jet penetration across the otherwise almost-parallel cross-flow. But the extent is not as great as the jet into infinite cross-flow cases of Fig. 5. Clearly visible are the turbulent eddies - these are very structured near the injection location, and appear to extend further downstream in the jet direction at lower values of the injection velocity. Downstream of the lateral jet entry location, in the lower part of the main flow, a sequence of what appears to be eddies shed behind the lateral jet obstacle. A sjmilar phenomenon was reported by Chassaing et al.3

The same sequence of lateral jet injection velocity ratios is given in Fig. 9, but now the strong downstream nozzle of area ratio 4 is located at $x / D=2$, which is one test-section diameter downstream of the lateral jet entry location. The effects of the nozzle seem to propagate upstream at most one-quarter the testsection diameter. Lateral jet penetration upstream of this location is unaffected by the presence of the nozzle. As the nozzle is approached, there is a dramatic increase in axial velocity and radial inflow toward the centerline. In the immediate environ of the nozzle, a small corner recirculation zone occurs just upstream near the confining walls. Stronger backflow is present now as compared to the open flow case. These details are seen in smoke most clearly at the lower lateral jet injection velocities. In the high injection velocity case, the jet path crosses the flow domain centerline, then retreats to the exit nozzle top expansion corner. The smoke tracer permits the top corner recirculation zone to be seen downstream of the nozzle in this case. An indication of the validity of the computations can be obtained by comparing predicted mean streamline patterns with the pathlines traced out by the soap bubbles in the flow visualization experiments. Generally, a relatively long exposure time of up to $15 \mathrm{~s}$ is used so as to identify many pathlines, and infer streamlines therefrom. Other interesting flow features can also be perceived. A sample flow visualization photograph is presented in Fig. 10 for each of the cases of jet to cross-flow velocity $R$ of $0,2,3,4,6$ and 8 in Parts (a) through ( $f$ ), respectively. These highlight the main flow and its changes due to the presence of the jet, as opposed to the earlier smoke photographs. In each case the peneteration and jet path are easily discernable, as the approaching parallel flow is deflected horizontally around the vertical injected jet. In this way the bubbles are taken out of the vertical light-curtain plane.

The flow visualization photographs in general provide an encouraging base of data for comparison with the computations. In all flowfields represented here, the calculation does a reasonable job of predicting the general flow patterns but it is expected that inaccuracies in detail will occur, as has been encountered by others in predicting recirculation zones with the standard $k-\varepsilon$ turbulence model, especially under swirl flow conditions. There are a number of possible reasons for this, but the most likely one is that a turbulence model whose basis and parameters are adequate for simple flow situations is not adequate to handle the more complicated swirling recirculation flow situation. Further detailed experimental work will attack this problem.

Velocity Profiles. Five-hole pitot probe measurements of time-mean velocity vectors in the vertical rx-plane through the lateral jet location provide further information on jet penetration and trajectory. Figures 11 and 12 show several radial traverses in the vertical plane with lateral 
a)

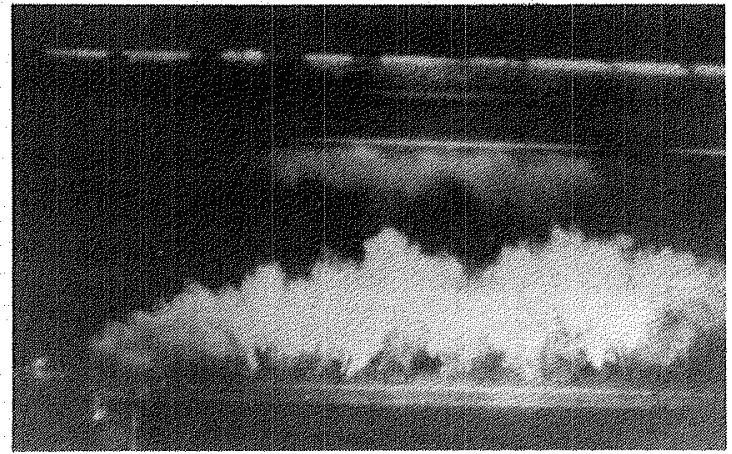

b)

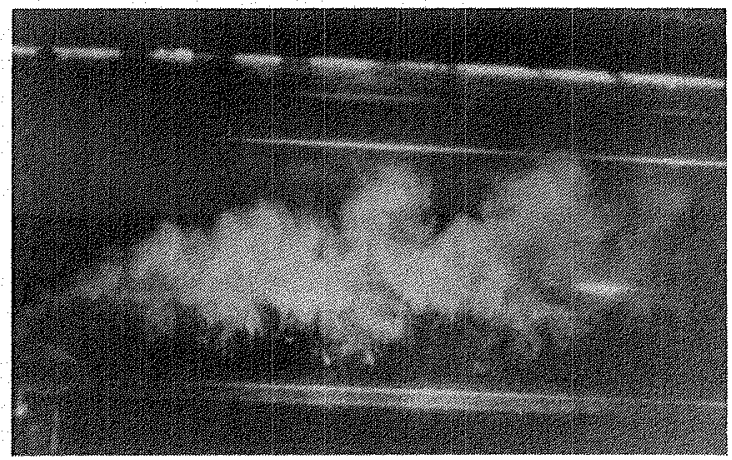

c)

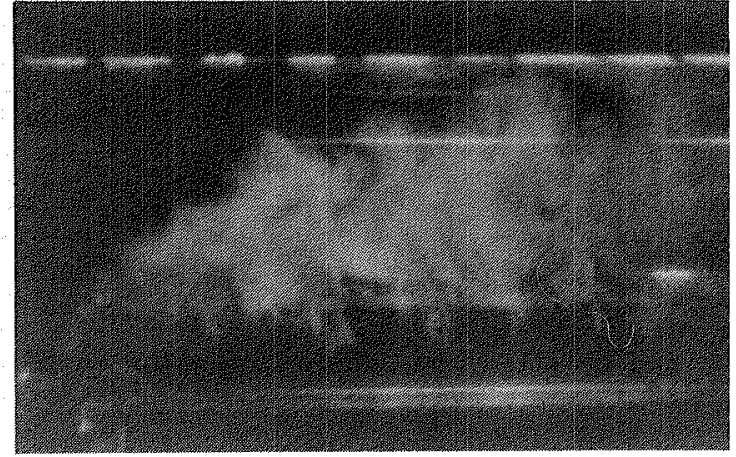

d)

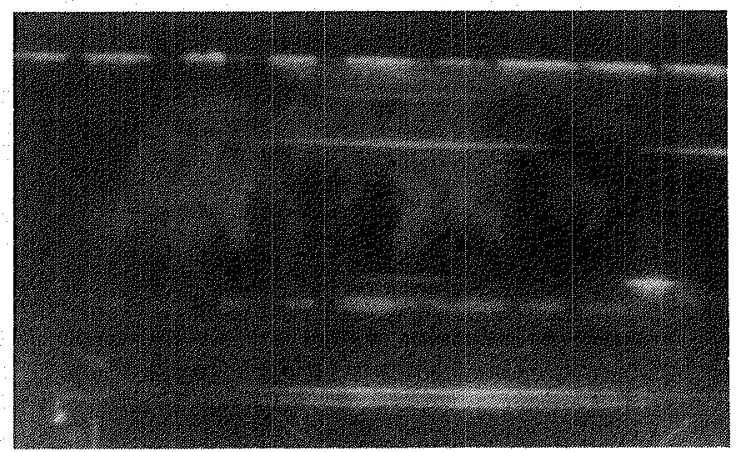

Fig. 8 Smoke visualization of lateral jet with velocity ratio $R$ of:

$\begin{array}{llll}\text { (a) } 2 & \text { (b) } 3 & \text { (c) } 4 & \text { (d) } 6\end{array}$ a)

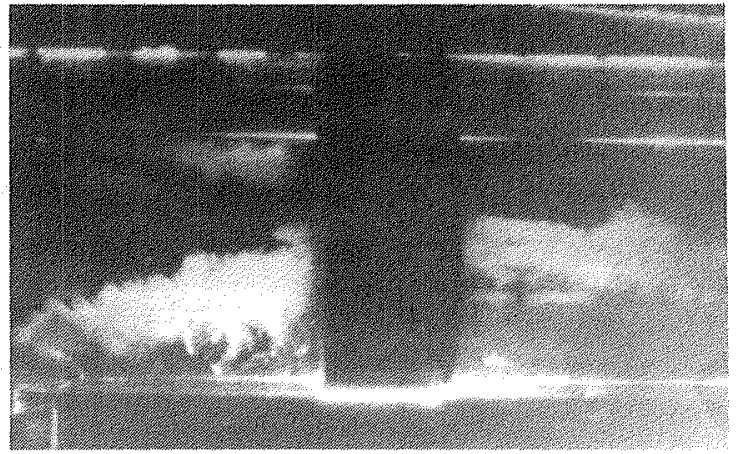

b)

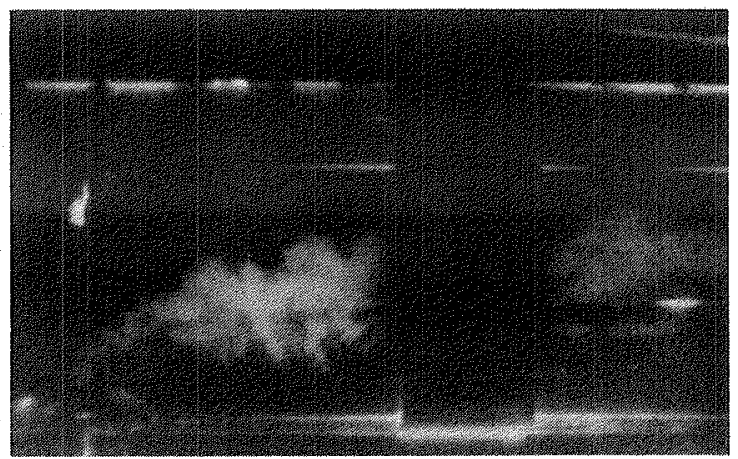

c)

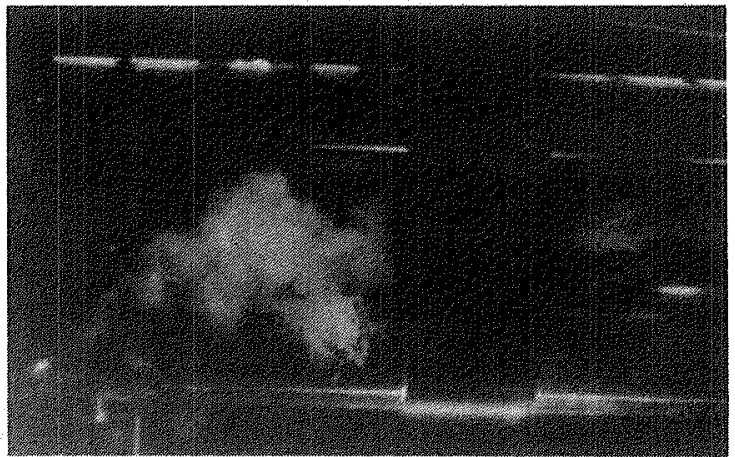

d)

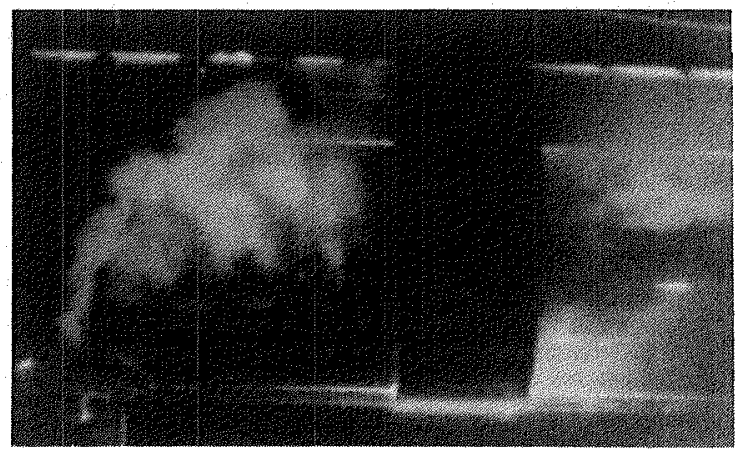

Fig. 9 Smoke visualization of lateral jet with downstream nozzle and velocity ratio $R$ of: (a) 2 (b) 3 (c) 4 (d) 6 . 
a)

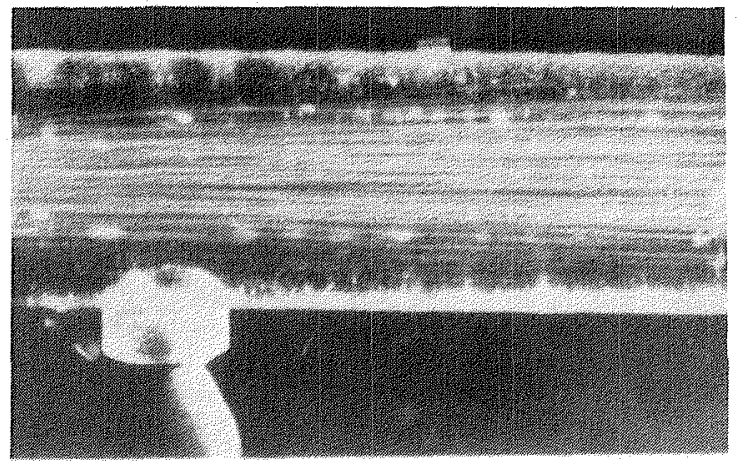

b)

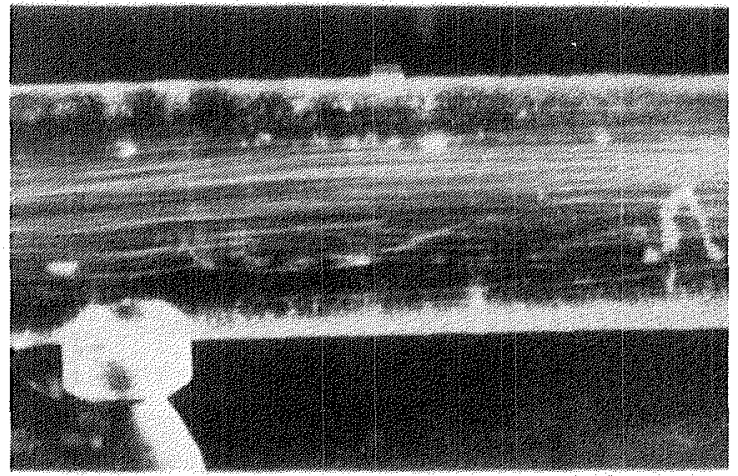

c)

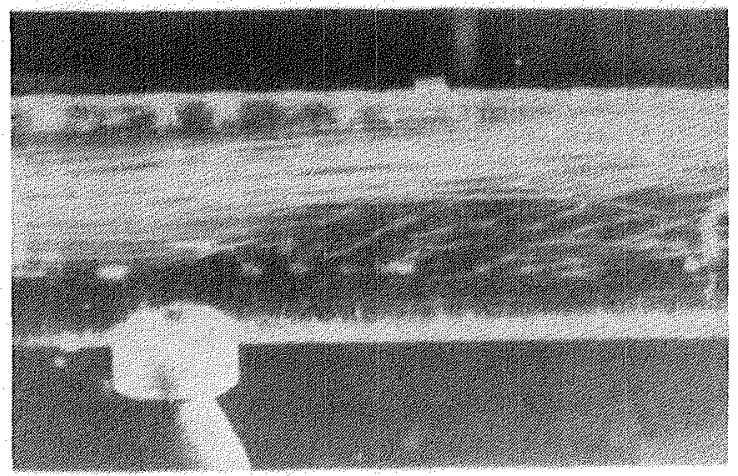

d)

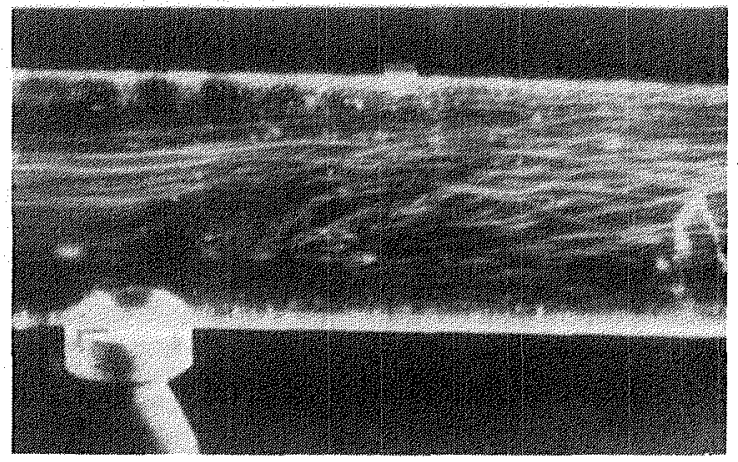

Fig. 10 Neutrally-buoyant helium-filled soap bubble visualization with velocity ratio $\mathrm{R}$ of:
(a) 0 (b) 2
(c) 3 (d) 4

e)

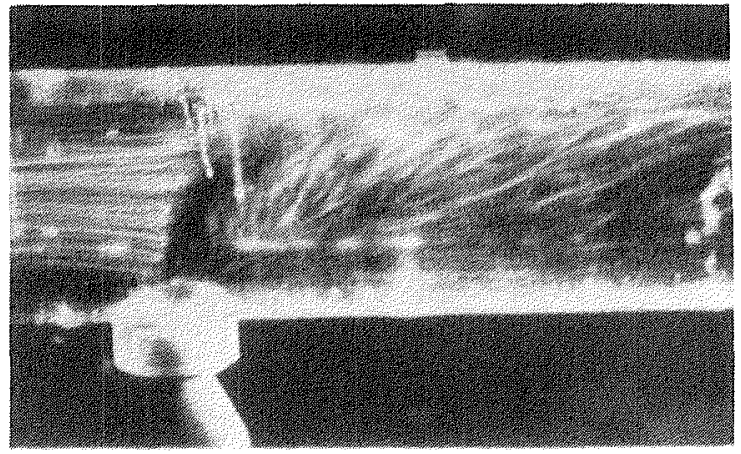

f)

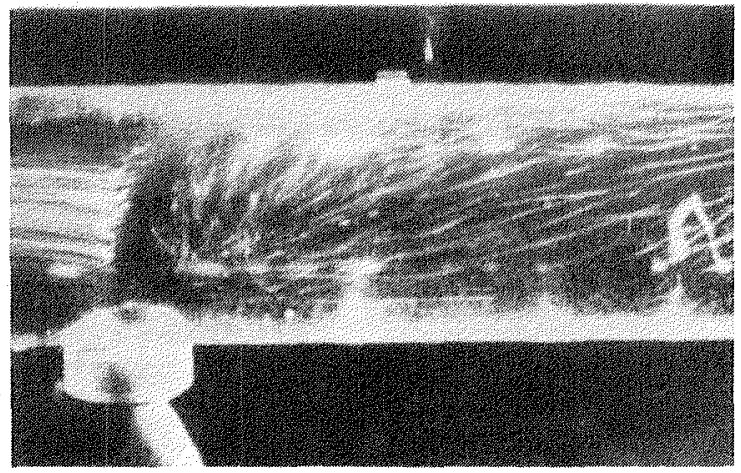

injection jet velocity $v_{j}$ equal to four times the inlet cross-flow velocity $u_{0}$. Figure 11 is the open-ended flow case; Figure 12 illustrates the downstream nozzle effect. These figures may be compared with similar flowfields measured ${ }^{11,15}$ and predicted ${ }^{12}$ elsewhere, in the absence of a dilution jet. Also shown on Figs. 10 and 11 is the location of the jet centerline. The upstream effect of the nozzle is seen to extend only to $x / D$ =1.75. The figures clarify jet penetration with and without the downstream nozzle, and confirm the ideas about the flow presented with Figs. 8 through 10 .

\section{Closure}

Fundamental experimental and theoretical research studies are being undertaken on the problem of lateral jet injection into typical combustor flowfields in the absence of combustion. Parameter variations to be systematically investigated include: lateral jet velocity, main inlet-to-combustor expansion ratio, combustor cross-flow inlet swirl strength, downstream contraction nozzle. The basic aim is to characterize the time-mean and turbulence flowfield with a variety of parameter settings, recommend appropriate turbulence model advances, and implement and exhibit results of flowfield predictions. The present study emphasizes the predictive capability of a fully three-dimensional evaluation using smoke and helium bubble flow visualization, and five-hole pitot probe time-mean velocity measurements. 


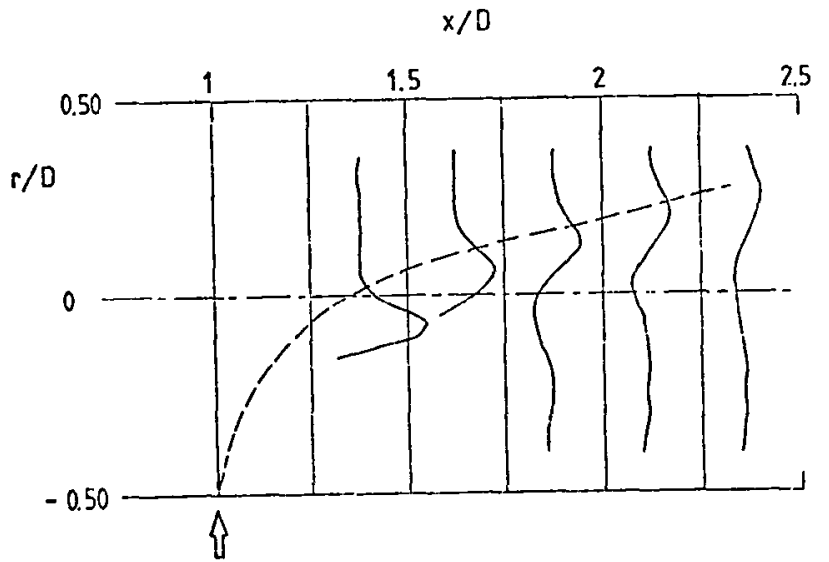

Fig. 11 Measured total velocity profiles with velocity ratio $R$ of 4 [---jet centerline].

\section{Acknowledgments}

The authors express their extreme gratitude to S. L. Mitchell and L. H. Ong for assistance with the computations and experiments.

\section{References}

1. Mellor, A. M. "Gas Turbine Engine Pollution." Prog. in Energy and Combustion Science, Vol. 1, 1976, pp. 111-133.

2. Lefebure, A. H. (ed.) "Gas Turbine Combustor Design Problems." Hemisphere-McGraw-Hill, New York, 1980.

3. Mularz, E. J. (ed.) "Combustion Fundamentals Research Conference," held at NASA Lewis Research Center, Cleveland, Ohio, Oct. 21-22, 1982.

4. Lefebvre, A. H. "Gas Turbine Combustion." McGraw-Hi11, New York, 1983.

5. Gupta, A. K., Lilley, D. G., and Syred, N. "Swirl Flows." Abacus Press, Tunbridge Wells, Engl and, 1984.

6. Gupta, A. K. and Lilley, D. G., "Flowfield Modeling and Diagnostics." Abacus Press, Tunbridge Wells, England, 1984.

7. Morel, T., "Comprehensive Design of Axisymmetric Wind Tunnel Contractions", ASME Paper 75-FE-17, Minneapolis, MN, May 5-7, 1975.

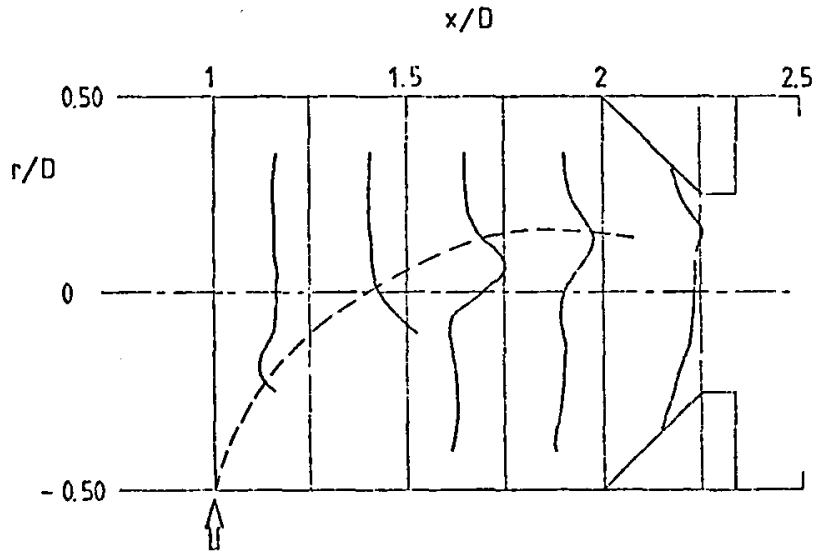

Fig. 12 Measured total velocity profiles with downstream nozzle and velocity ratio $R$ of 4 [-- jet centerline].
8. Rhode, D. L., "Predictions and Measurements of Isothermal Flowfields in Axisymmetric Combustor Geometries", Ph.D. Thesis, Oklahoma State University, Stillwater, Okla., Dec. 1981 .

9. Janjua, S. I., "Turbulence Measurements in a Complex Flowfield Using a Six-Orientation Hot-Wire Probe Technique", M.S. Thesis, Oklahoma State University, Stillwater, Okla., Dec. 1981.

10. Yoon, H. K., "Five-Hole Pitot Probe Time-Mean Velocity Measurements in Confined Swirling Flows", M.S. Thesis, Oklahoma State University, Stillwater, Okla., July, 1982.

11. Jackson, T. W., "Turbulence Characteristics of Swirling Flowfields", Ph.D. Thesis, Oklahoma State University, Stillwater, OK, Dec. 1983.

12. Abujelala, M. T., "Confined Turbulent Swirling Recirculating Flow Predictions", Ph.D. Thesis, Oklahoma State University, Stillwater, OK, May 1984.

13. Sander, G. F., "Axial Vane-Type Swirler Performance Characteristics", M.S. Thesis, Oklahoma State University, Stillwater, OK, July 1983.

14. McKillop, B. E., "Turbulence Measurenents in a Complex Flowfield Using a Crossed HotWire", M.S. Thesis, Oklahoma State University, Stillwater, OK, July 1983. 
15. Scharrer, G. L., "Five-Hole Pitot Probe Measurements of Swirl, Confinement and Nozzle Effects on Confined Turbulent Flow", M.S. Thesis, Oklahoma State University, Stillwater, OK, May 1984.

16. Rhode, D. L.., Lilley, D. G., and McLaughl in, $D$. K., "On the Prediction of Swirling Flowfields Found in Axisymmetric Combustor Geometries", ASME Journal of Fluids Engineering, Vol. 104, Sept. 1982, pp. 378384.

17. Rhode, D. L., Lilley, D. G., and McLaughlin, D. K., "Mean Flowfields in Axisymmetric Combustor Geometries with Swirl", AIAA Journal, Vol. 21, No. 4, April 1983, pp. 593600.

18. Yoon, H. K., and Lilley, D. G., "Five-Hole Pitot Probe Time-Mean Velocity Measurements in Confined Swirling Flows", Paper AIAA-830315, Reno, Nevada, January 10-13, 1983.

19. Abujelala, M. T., and Lilley, D. G., "Confined Swirling Flow Predictions", Paper AlAA-83-0316, Reno, Nevada, Jan. 10-13, 1983.

20. Sander, G. F., and Lilley, D. G., "The Performance of an Annular Vane Swirler", Paper AIAA-83-1326, Seattle, Wash., June 27$29,1983$.

21. Janjua, S. I., McLaughlin, D. K., Jackson, T. W., and Lilley, D. G., "Turbulence Measurements in a Confined Jet Using a SixOrientation Hot-Wire Probe Technique", Paper AIAA-82-1262, Cleveland, $\mathrm{OH}$, June 21-23, 1982, AIAA Journal, 1983 (in press).

22. Jackson, T. W., and Lilley, D. G, "SingleWire Swirl Flow Turbulence Measurements", Paper AIAA-83-1202, Seattle, Wash., June 27$29,1983$.

23. Lilley, D. G., and Rhode, D. L., "A Computer Code for Swirling Turbulent Axisymmetric Recirculation Flows in Practical Isothermal Combustor Geometries", NASA CR-3442, Feb. 1982.

24. Samples, J. W. "Prediction of Axisymmetric Chemically-Reacting Combustor Flowfields." Ph.D. Thesis, OKlahoma State University, Stillwater, Okla., May 1983.

25. Busnaina, A. A. "Transient Three-Dimensional Predictions of Turbulent Flows in Cylindrical and Cartesian Coordinate Systems. Ph.D. Thesis, Oklahoma State University, Stillwater, Okla., July 1983.

26. Schetz, J. A., "Injection and Mixing in Turbulent Flow", Prog. in Astro. and Aero., AIAA, Vol. $68,1980$.

27. Callaghan, E. E, and Ruggeri, R. S., "Investigation of the Penetration of an Air Jet Directed Perpendicularly to an Air Stream", NACA TN 1615, 1948.

28. Ruggeri, R. S., Callaghan, E. E., and Bowden, D. T., "Penetration of Air Jets Issuing from Circular, Square and Elliptical Orifices
Directed Normally to an Air Stream", NACA TN 2019, Feb. 1950.

29. Ruggeri, R. S., "General Correlations of Temperature Profiles Downstream of a Heated Air Jet Directed at Various Angles to an Air Stream", NACA TN 2855, 1952.

30. Jordinson, R., "Flow in a Jet Directed Normal to the Wind", Aero. Res. Council $R$ and $M$ No. $3074,1958$.

31. Keffer, J. F., and Baines, W. D., "The Round Turbulent Jet in a Cross Wind", ASME Journal of Fluid Mechanics, Vol. 15, 1963, p. 481.

32. Pratt, B. D., and Baines, W. D., "Profiles of the Round Turbulent Jet in a Cross Flow", Proceedings of ASCE, Journal of the Hydraulics Division, Nov. 1967, pp. 56-63.

33. Norgren, C. T., and Humenik, F. M., "Dilution Jet Mixing Study for Gas-Turbine Combustors", NASA TN-D-4695, Aug. 1968.

34. Pratt, B. D., and Keffer, J. F., "Deflected Turbulent Jet Flows", Journal of Applied Mechanics, 1971, pp. 756-758.

35. Campbell, J. F., and Schetz, J. A., "Analys is of Injection of a Heated Turbulent Jet into a Cross-flow", NASA TR R-413, Dec. 1973.

36. Kamotani, Y., and Greber, I., "Experiments on Confined Turbulent Jets in Cross Flow", NASA CR-2392, March 1974.

37. Holdeman, J. D., and Walker, R. E., "Mixing of a Row of Jets with a Confined Crossflow", AIAA Journal, Vol. 15, No. 2, Feb. 1977, pp. 243-249.

38. Chassaing, P., George, J., Claria, A., and Sananes, F., "Physical Characteristics of Subsonic Jets in a Cross-Stream", Journal of Fluid Mechanics, Vol. 62, 1974, p. 41-64..

39. Rathgeber, D. E., and Becker, H. A., "Mixing Between a Round Jet and a Transverse Pipe Flow", Proceedings of lst symposium on Turbulent Flows, Penn State University, 1977.

40. Krausche, D., Fearn, R. L., and Weston, R. P., "Round Jet in a Cross Flow: Influence of Injection Angle on Vortex Properties", AIAA Journal, Vol. 16, June 1978, pp. 636-637.

41. Crabb, D., Durao, D. F. G., and Whitelaw, J. W., "A Round Jet Normal to a Cross-Flow", ASME Trans., Vol. 103, 1981.

42. Holdeman, J. D., "Dilution Zone Mixing", Presented at Combustion Fundamentals Research Conference, NASA Lewis Research Center, Cleveland, Ohio, 0ct. 21-22, 1982, pp. 106118.

43. Ferrel1, G. B., Ph.D. Studies in Progress, 1983.

44. Srinivasan, R., Bernfeld, A., and Mongia, $H_{.}$ C. "Dilution Jet Mixing Program Phase I Report." NASA CR-168031, Nov. 1982. 
45. Clau's', 'R. W., "Analytical Calculation of a Single Jet in Cross-Flow and Comparison with Experiment." Paper AIAA-83-0238, Reno, Nevada, Jan. 10-13, 1983.

46. Launder, B. E., and Spalding, D. B., "The Numerical Computation of Turbulent Flows", Comp. Methods in Appl. Mech. and Engrg., Vol. 3, Mar. 1974, pp. 269-289.

47. Hirt, C. W., Nichols, B. D., and Romero, N. C., "SOLA - A Numerical Solution Algorithm for Transient Fluid Flows", Report LA-5852, Los Alamos Scientific Laboratory, Los Alamos, NM, 1975.

48. Patankar, S. V., Numerical Heat Transfer and Fluid Flow, Hemisphere-McGraw-Hill, New York, 1980.

49. Patankar, S. V., Basu, D. K., and Alpay, S. A., "Prediction of the Three-Dimensional Velocity Field of a Deflected Turbulent Jet", Journal of Fluids Engineering, Vol. 99, 1977, pp. 758-762.
50. Ramsey, J. W., and Goldstein, R. S., "Interaction of a Heated Jet with Deflecting Stream", NASA CR-72613, 4TL TR-92, April 1970.

51. Serag-Eldin, M. A., and Spalding, D. B., "Computation of Three-Dimensional Gas Turbine Combustion Chamber Flows", ASME 78-GT-142, London, England, 1978.

52. Swithenbank, J., Turan, A., and Felton, P. G., "3-Dimensional 2-Phase Mathematical Modeling of Gas Turbine Combustors", project SQUID (ONR) Workshop, Gas Turbine Combustor Design Problems, meeting held at Purdue University, W. Lafayette, Indiana, May 31June $1,1978$.

53. Boysan, F., Ayers, W. H., Swithenbank, J., and Pan, Z., Three-Dimensional Model of Spray Combustion in Gas Turbine Combustors", AIAA81-0324, 19th Aerospace Sciences Meeting, St. Louis, Missouri, 1981.

54. Lilley, D. G., "Flowfield Modeling in Practical Combustors: A Review", Journal of Energy, Vol. 3, No. 4, July-August 1979, pp. 193-210. 
APPENDIX B

FLOW VISUALIZATION OF LATERAL JET INJECTION

INTO SWIRLING CROSSFLOW

AIAA-85-0059 
FLOW VISUALIZATION OF LATERAL JET INUECTION INTO SWIRLING CROSSFLOW

\author{
G. B. Ferrell*, K. Aoki** and D. G. Lilley*** \\ Oklahoma State University \\ Stillwater, 0klahoma 74078
}

\section{ABSTRACT}

Flow visualization experiments have been conducted to characterize the time-mean flowfield of a deflected turbulent jet in a confining cylindrical crossflow. Jet-to-crossflow velocity ratios of 2, 4, and 6 were investigated, under crossflow inlet swirler vane angles of 0 (swirler removed), 45 and 70 degrees. Smoke, neutrallybuoyant helium-filled soap bubbles, and multi-spark flow visualization were employed to highlight interesting features of the deflected jet, as well as the trajectory and spread pattern of the jet. Gross flowfield characterization was obtained for a range of lateral jet-to-crossflow velocity ratios and a range of inlet swirl strengths in the main flow. The flow visualization results agree well with the measurements obtained elsewhere with the six-orientation single hot-wire method.

\section{NOMENCLATURE}

\begin{tabular}{l}
$A$ \\
$D$ \\
$Q$ \\
$R$ \\
$u, v, w$ \\
$x, r, \theta$ \\
$\phi$ \\
Subscripts \\
\hline$b$ \\
$c, 0$ \\
$j$ \\
$n$ \\
$r$
\end{tabular}

Cross-sectional area

Test-section diameter

Volume flowrate

Jet-to-crossflow velocity ratio $v_{j} / u_{0}$

Axial, radial and swirl velocity

components

Axial, radial and azimuthal

coordinates

Swirl vane angle

Relating to bubbles

Relating to crossflow

Relating to lateral jet

Relating to nozzle

Relating to total

\section{INTRODUCTION}

\subsection{The Lateral Jet Problem}

Almost all flow phenomena of practical engineering interest are turbulent and do not lend themseives easily to experimental, analytical or numerical investigation. Deflected turbulent jets are no exception, involving fully three-dimensional flow phenomena. Power plant chimney plumes, cooling holes ejecting air from turbine blades, and the airflow around V/STOL aircraft in transition

* Graduate Student, School of Mechanical and Aerospace Engineering, Member AIAA. Presently Assistant Professor, Department of Mechanical Engineering, Texas Tech University, Lubbock, Texas.

** Associate Professor, Department of Mechanical Engineering, Tokai University, Japan, Member AIAA.

*** Professor, School of Mechanical and Aerospace Engineering, Associate Fellow AIAA.

This paper is declared a work of the U.S.

Government and therefore is in the public domain. flight are examples of deflected turbulent jets. The present study emphasizes lateral injected jets into tubular crossflow which may also possess swirl - a situation occurring in gas turbine combustion chambers, and a more complicated example of fully 3-D flowfields. Gas-turbine combustor designers utilize this mixing phenomena to promote rapid combustion and evenly mix the products of combustion with cooler injected air, accomplishing this in a compact space. ${ }^{-3}$

In gas turbine combustion chambers, most fuel burning takes place in the primary zone, the secondary zone is where combustion is almost completed, and the dilution zone is where further temperature reduction and uniformity is achieved. In both can and annular combustors, lateral jets of cooler air through round holes penetrate the flowfield. Some of these jets amalgamate with the swirl-induced central recirculation region of the primary zone and provide sufficient additional air for stoichiometric conditions. Other lateral jets provide additional air to help complete combustion in the secondary zone and to cool and evenly mix the products in the dilution zone in preparation for introduction of the flow to the turbine. Similar problems arise in ramjet combustors. Clearly the turbulent reacting flowfield is fully three-dimensional; the combustion designer has a formidable problem in aerothermochemistry, and the research and development task is to provide a route which leads to the accomplishment of design objectives more quickly and less expensively than current practice permits.

\section{2 objectives}

The focus of the present study is to present the main characteristics of a deflected turbulent jet, entering laterally into confined tubular crossflow which may also possess swirl. Part of an ongoing research effort at Oklahoma State University, the ultimate aim is to provide a data base for turbulence model advances used by combustor designers. The specific objective of this investigation was the use of several flow visualization techniques to highlight the important features and structures of the deflected jet. Neutrally-buoyant helium-filled soap bubbles, smoke, and multi-sparks were used. Flow visualization was used to characterize one deflected jet entering laterally into crossflow without swirl (swirler removed) and with inlet crossflow swirler vane angles of 45 and 70 degrees.

\subsection{Background}

Earlier studies at OSU on the flowfield without lateral injection jets are summarized by Lilley. ${ }^{4}$ They included experimental and theoretical research under low speed, nonreacting, turbulent, swirling flow conditions. The flow enters the test section and proceeds into a larger chamber (the expansion $\mathrm{D} / \mathrm{d}=2,1.5$ or 1 ) via a sudden or gradual expansion (side-wall angle $\alpha=$ 
90 and 45 degrees). A weak or strong nozzle may be positioned downstream to form a contraction exit to the test section. Inlet swirl vanes are adjustable to a variety of vane angles with values of $\phi=0$ (swirler removed), 38, 45, 60 and 70 degrees being emphasized. The objective was ti jetermine the effect of these parameters on isothermal flowfield patterns, time-mean velocities and turbulence quantities, and to establish an improved simulation in the form of a computer prediction code equipped with a suitable turbulence model. Helium bubble flow visualization, five-hole pitot probe time-mean velocity measurements, and one-wire and two-wire hot-wire normal and shear stress turbulence data were obtained in the experimental program. Turbulence modeling deductions and flowfield predictions were made via a 2-D axisymmetric technique.

A research program is now underway which deals both experimentally and theoretically with the problem of primary and dilution lateral jet injection into typical combustor flowfields in the absence of combustion. Parameter variations to be systematically investigated include: lateral jet velocity, combustor crossflow inlet swirl strength, and downstream contraction nozzle. The basic aim is to characterize the time-mean and turbulence flowfield with a variety of parameter settings, recommend appropriate turbulence model advances, and implement and exhibit results of flowfield predictions. Preliminary results were given in Ref. 5, together with a review of jets in crossflow. A recent Ph.D. thesis by Ferrell' includes flow visualization, multi-orientation single-wire hot-wire measurements, and an extensive review of this phenomenon. Noteworthy earlier studies include Chassaing et al, 7 Rathgeber and Becker, 8 Crabb et al ${ }^{9}$ and Holdeman et al.10 Recent textbooks review and present the progress which has been made. Schetzil concentrates on the physics and modeling of injection and mixing of turbulent flows; Lefebvre 1 acknowledges that the phenomena are of paramount importance in the combustion and dilution zones, and presents recent progress and its relevance to combustor design requirements.

Many methods have been used in the manifestation of flowfields, and the text by Merzkirchi2 on flow visualization is commendable. Smoke injection, helium bubble injection, and tuft grid method have been used in earlier studies without any lateral jets, 13 and recently with one and two lateral jets. 14 Smoke and bubbles are used in the present study, which also uses a multi-spark visualization method, from which quantitative velocity deductions are also possible. The method is described by Nakayama et al15,16 and has recently been applied to the present geometry for a wide variety of parameter variations. 17 The spark generation method has been used in the past for boundary layer study, 18 for hypersonic velocity measurements, 19 and for visualization of wakes behind ballistic projectiles. 20

\subsection{Outline of the Paper}

In the present paper, a flow visualization study is made of the gross flowfield characteristics. Section 2 describes the experimental facility. The flow visualization techniques used are expounded upon in Section 3 , including bubbles, smoke and sparks. Results are presented and discussed in Section 4, and the final closure summarizes the achievements.

\section{EXPERIMENTAL FACILITY}

\subsection{Wind Tunnel}

The test facility, dominated by the wind tunnel, is shown schematically in Figure 1. Air is induced to flow through a large foam inlet filter by an axial fan. The fan is connected by way of belts and pulleys to a seven-horsepower U.S. Motor varidrive. The varidrive permits the fan speed to be adjusted from 500 to 2850 RPM. The air is then forced into an expanding area section, where multiple 20 -mesh screens and a section of straws are employed to straighten the flow and significantly lower the turbulence intensity.

Before introduction to the test section, the air flows through an axisymetric nozzle with an area reduction ratio of 25 . The nozzle was built with a matching cubic radial profile, according to the method described by Morel21. The objective of this nozzle design is to produce a low turbulence level uniform velocity profile before introduction to the test section, with minimum adverse pressure gradient in the boundary layer. The exit throat diameter of the wind tunnel is approximately $15 \mathrm{~cm}$.

At the throat of the tunnel and before introduction to the test section, a variable-angle swirler may be optionally fitted. The swirler consists of ten flat blades (with pitch-to-chord ratio 0.68 ) which may be individually adjusted to any angle from 0 to 70 degrees. Sander 22 provides detailed information and measurements related to the swirler performi...e and swirler exit velocity profiles on this facility.

\subsection{Test Section and Dilution Jet}

The test section consists of a clear acrylic tube approximately $90 \mathrm{~cm}$ in length attached to the wind tunnel throat. Standard commercial acrylic tube is used with $15.24 \mathrm{~cm}$ outside diameter, 0.318 $\mathrm{cm}$ wall thickness. The inside diameter is therefore $14.61 \mathrm{~cm}$ with an observed variation of + $0.05 \mathrm{~cm}$. To adapt the test section to the wind tunnel throat (inside diameter $15 \mathrm{~cm}$ ), an adaptor section was machined to provide a smooth transition from wind tunnel throat to test section. The test section, See Figure 2, has the dilution jet inlet located at $x / D=1.00$ where $x$ is measured from the tube inlet.

Laboratory compressed air at 6 to 7 atmospheres gauge pressure is used to supply the dilution jet. For stability, the supply air lines are large and are routed through two line regulators with an intermediate tank (volume approximately $0.006 \mathrm{~m}^{3}$ ) to dampen line oscillations. The second regulator is used to meter the flow rate. After the second regulator, the air is routed through a Fisher and Porter model $10 \mathrm{~A} 1735 \mathrm{~A}$ rotameter for monitoring of the volume flow before introduction to the dilution jet. The dilution jet assembly, see Figure 3 , consists of a stagnation chamber, flow straightening section, and the jet nozzle. The stagnation chamber is constructed from $15 \mathrm{~cm}$ inside diameter aluminum pipe and filled with plastic household scrub pads to evenly distribute the internal flow. A hemispherically-shaped screen and radiused 


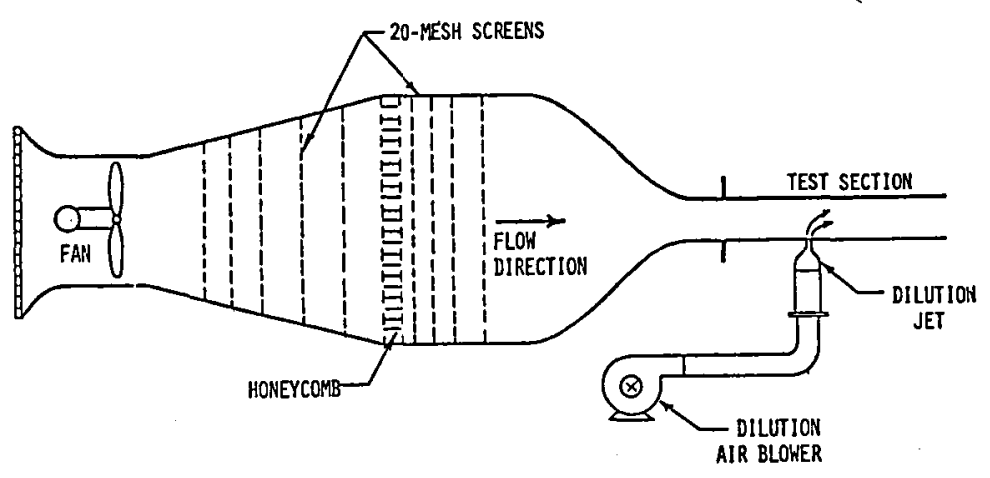

Fig. 1. Schematic of Experimental Facility

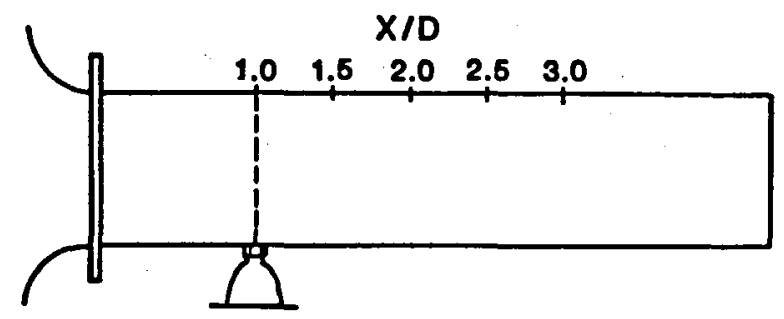

Fig. 2. Test Section Geometry

transition smooth the flow into the flow straightening section. Here the air flows through four brass screens for turbulence reduction. The nozzle is designed according to the method suggested by Morel 21 and is constructed of fiberglass. The nozzle diameter is 0.10 of the test-section diameter for a crossflow to jet area ratio $A_{c} / A_{j}=100$. Once assembled, the dflution jet is attached to the air line coming from the rotameter and the nozzle is pressed into a special acrylic adaptor which is permanently attached to the test section.

\section{FLOK VISUALIZATION}

Flow visualization is used for primary identification and characterization of the flowfield, with three techniques being used. Bubbles, because of their reflective qualities and neutral buoyancy in the airflow, provide an excellent medium to determine the paths of the flow trajectories. Smoke, because of its low comparative density and its tendency to mix well in the flow, makes an excellent medium to follow the flow and to accent the turbulent paths and recirculation zones in the flow. A more novel flow visualization technique employed in these investigations is the multi-spark method. With this technique, an ionized path is used to determine the relative velocity change from the position of the electrodes. The basic rig set up is the same for all three flow visualization techniques, however differences occur in the type and quantity of lighting units, the camera time settings and light exposure times.

\subsection{Bubble Flow Visualization}

The bubble generator is manufactured by Sage Action, Inc. It generates about 100 bubbles per second. The helium regulator range is $0-207 \mathrm{kPa}$ and flows at a maximum rate of $2.67 \times 10^{-6} \mathrm{~m}^{3} / \mathrm{s}$. The maximum bubble solution flow rate is $2.50 \times$

$10^{-8} \mathrm{~m} / \mathrm{s}$ and the maximum air flow rate is $2.60 \times$ $10^{-4} \mathrm{~m} / \mathrm{s}$. A helium tank and an air line both with associated pressure gauges are connected to the bubble generator. The SAI bubble flow solution (BFS) is inserted directly into a reservoir in the bubble generator itself. The soap solution is pumped out of the reservoir via helium. Three lines from the bubble generator are attached to the bubble injector head. The head itself typically consists of three concentric tubes. The center one for helium, the middle annulus is for soap and the outside annulus is for air. Each line may be regulated by valves on the top of the bubble generator. A hole in the sleeve directly below the nozzle is where the injector head is inserted to inject bubbles parallel to the flow.

The maximum bubble flow rate is $15.24 \mathrm{~cm}^{3} / \mathrm{s}$. The slowest nozzle velocity is about $4.2 \mathrm{~m} / \mathrm{s}$. From the equation $Q_{T}=Q_{B}+Q_{b}$ where: where $Q_{T}=$ total volume flowrate, $Q_{n}=b$ volume flowrate of the nozzle, and $Q_{b}=$ volume flowrate of the bubbles, it was found that $Q_{b} / Q_{n}=0.0042$; approximately one half of one percent. Hence the bubble flowrate has insignificant effect on the nozzle flowrate. The injector heads are also streamlined to minimize turbulent effects.

The lighting for bubble flow visualization is approximately $3 \mathrm{~m}$ downstream of the test section. A light curtain dial provides light curtains from 


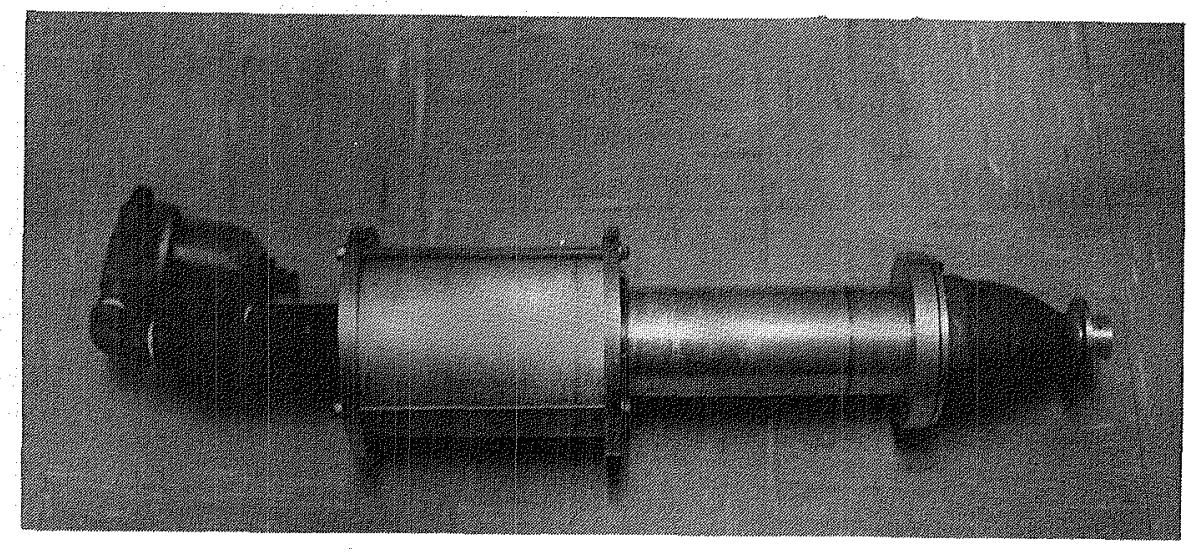

Fig. 3. Dilution Jet (Photo)

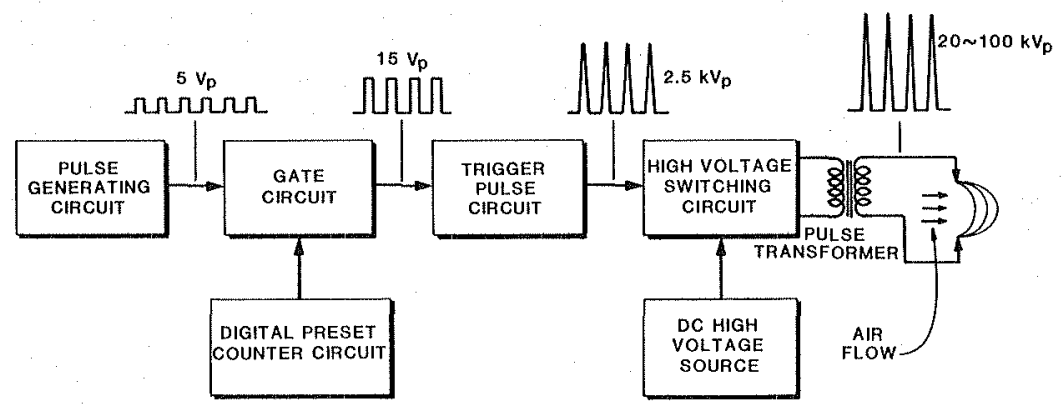

Fig. 4. Spark-Gap Equipnent Schematic

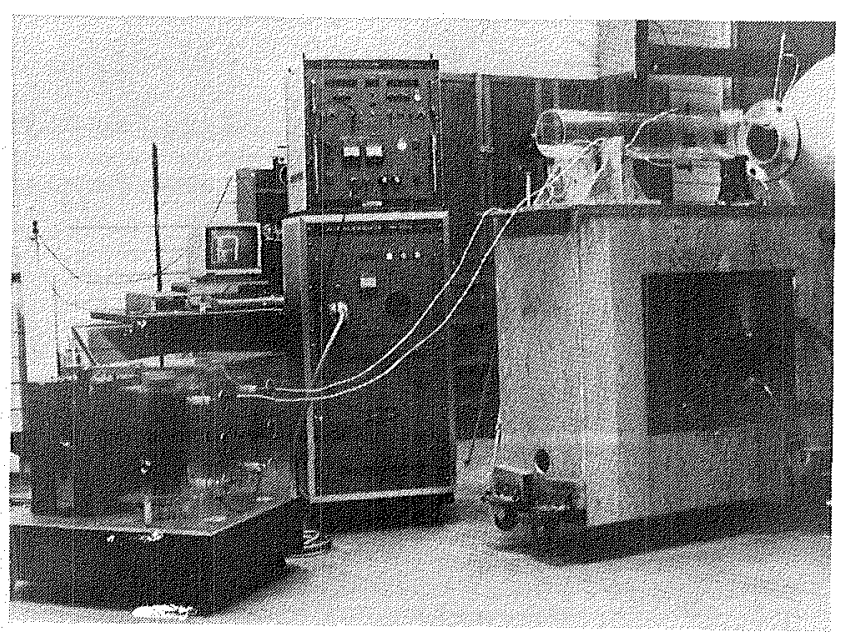

Fig. 5. Spark-Gap Equipment (Photo) 


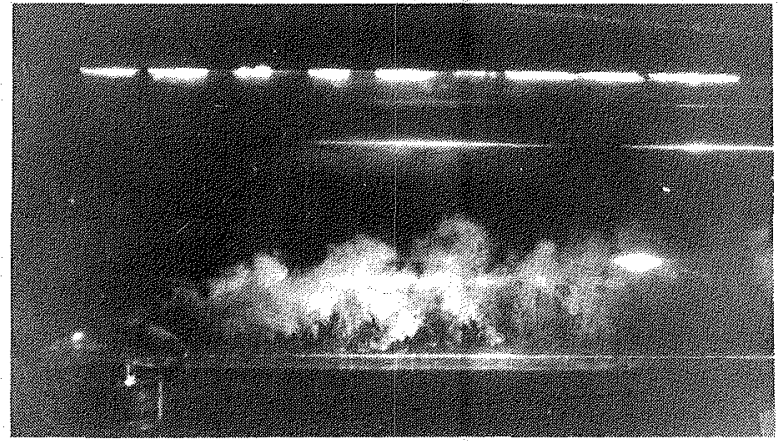

(a) $\mathrm{R}=2.0$

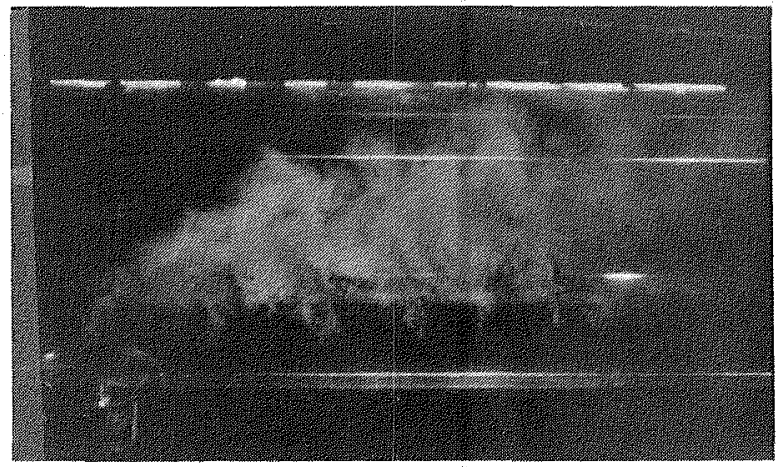

(b) $R=4.0$

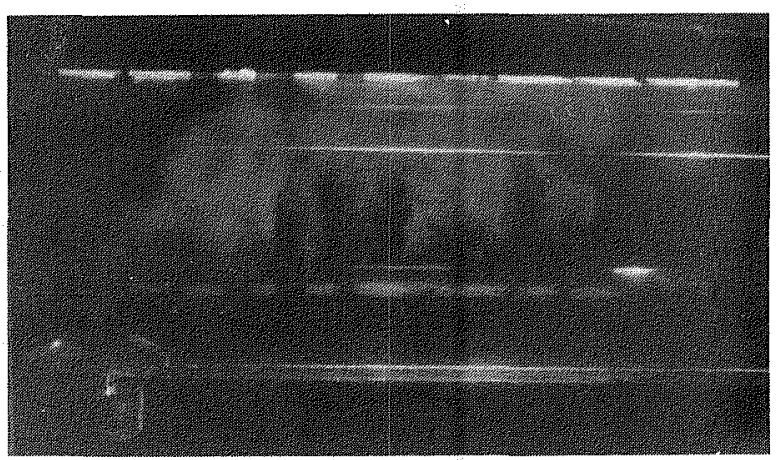

(c) $R=6.0$

;ig. 6. Smoke Flow Visualization for Jet-toCrossflow Velocity Ratio $R=2.0,4.0,6.0$
$0-1.5 \mathrm{~cm}$ wide through an adjustable slit and may be positioned to emit light angles from 0 to 360 degrees. The lighting is on throughout the photography session and the exposure time is determined by the camera settings. The camera used is a Minolta SRI 200. The films used include Kodak Tri-X Pan 400 ASA black and white, I1ford 400 ASA black and white, and Kodak color 1000 ASA film, with all of these giving excellent results. The camera is positioned approximately $0.5 \mathrm{~m}$ laterally from the test section and supported by a tripod. A low F-stop of 2 is used for maximum light intake; the exposure time is set on $B$ for a 5-second count. These settings were chosen after much testing to accentuate the bubble streaklines illustrating the flow trajectories.

\subsection{Smoke Flow Visualization}

The smoke generator consists of a heating coil wrapped in steel wool and surrounded by a metal box with a drip tray in the bottom of the box to catch excess oil which may be drained out through a drain plug. Attached to the metal box is a thermocouple which runs to a temperature gauge outside the generator. Internal temperatures may then be monitored. Experiments have shown that a temperature gauge reading between 250 and $300^{\circ} \mathrm{C}$ produces the optimum amount of smoke for flow visualization. The temperature is not allowed to exceed $350^{\circ} \mathrm{C}$ to prevent gasket damage. The temperature is adjusted through a rheostat which is generally turned up to $50 \%$ power and then reduced to $30 \%$ power for temperature stabilization. A valve above the generator may be opened to drip more oil onto the heating coil as needed for smoke generation. The air-line which is adjusted by the rotameter runs through the smoke generator and up through the nozzle forcing the smoke through the identical path. A few drops of $0 i 1$ produces a considerable amount of smoke and therefore has negligible effect on the previously monitored flowrate.

The lighting device used for flow visualization consists of two commercial flash units. One unit is a Vivitar 2800 and the other a Sunpack 422D. The flash times may be adjusted on the units and the F-stop varied according to the distance of the camera from the flashes. Experimental results have shown that $F-s t o p=4$ and flash time $=1 / 2000 \mathrm{~s}$ with camera settings of $\mathrm{F}-$ stop equal 2 and exposure time from 0.125 to $0.5 \mathrm{~s}$ produce the best pictures highlighting the flowfield features. The flash units are in a black box with a slit parallel to the test section and placed directly beneath and touching the test section. The positioning was chosen to minimize glare and maximize lighting through a vertical slit of light accenting a vertical cross-section of the flowfield.

\subsection{Spark-Gap Flow Visualization}

The spark-gap equipment schematic and configuration is shown in Figures 4 and 5 . The pulse generating circuit and pulse transformer is manufactured by Sugawara Laboratories, Inc., Tokyo. The equipment is capable of producing pulse trains of up to 200 pulses at frequencies from 1 $\mathrm{kHz}$ to $75 \mathrm{kHz}$. The output energy of the pulse. is 0.05 to 0.5 joules at voltages from $20 \mathrm{kV}$ to 250 $\mathrm{kV}$. As used in the present study, the electrodes are placed on opposite sides of the test section, 
typically one electrode above and one below the test section with a $15 \mathrm{~cm}$ spark gap. Approximately 40 sparks are used with $0.5 \mathrm{~J} /$ spark at a voltage of $100 \mathrm{kV}$. Each spark pulse duration is approximately $1 \mu \mathrm{s}$; time between pulses is approximately $1 \mathrm{~ms}$.

When a high voltage source is sparked across an air gap, an ionized path is created. Subsequent sparks will follow the current position of this low-resistance ionized path. By judicious placement of the electrodes in the wall boundary layer, where there is essentially zero velocity (next to wall), several discharges can follow the original ionized path as it moves with the fluid. It is necessary to have a low-conductivity test section material (for example, acrylic) so as not to interfere with spark paths. The spark itself provides sufficient lighting for photographs. One camera (side view) is used for photographs with zero swirl. Two cameras (side and end view) are used simultaneously in the swirl crossflow cases to give added perspective to the three-dimensional features of the resulting flowfield.

\section{RESULTS AND DISCUSSION}

\subsection{Smoke}

Figure 6 shows very short time exposures of smoke tracing the extent of the deflected jet with jet-to-crossflow velocity ratio $R$ of 2,4 , and 6 in parts (a) through (c) respectively. The exposure time is of the order of $1 \times 10^{-4} \mathrm{~s}$, and vertical slit lighting is obtained with two commercial flash units. The camera is positioned to the side so as to obtain a view of the vertical $r x-p l a n e$ in the flowfield. Notice that as the deflected jet velocity increases, so also does the jet penetration across the otherwise almost-parallel crossflow. clearly visible are the turbulent eddies - these are very structured near the injection location, and appear to extend further downstream in the jet direction at lower values of the injection velocity. Downstream of the deflected jet entry location, in the lower part of the main flow, a sequence of what appears to be eddies shed behind the lateral jet obstacle. A similar phenomenon was reported by Chassaing et a1.7

\subsection{Bubbles}

Figures 7,8 and 9 present long-time exposures of bubbles tracing the extent of the deflected jet with jet-to-crossflow velocity ratio $R$ of 2,4 , and 6 in each figure respectively. Each figure presents swirl angles of 0,45 , and 70 degrees in parts (a), (b), and (c) respectively. As with smoke tracing, the bubbles show the increase of penetration with increase in $R$. The bubbles, however, show the time-mean boundaries of the deflected jet. Clearly evident is the lack of penetration in the $R=2$ case; the $R=4$ case crosses the test-section centerline at approximately $x / D=1.4$ where the jet enters at $x / D$ $=1.00$ and then continues down the test section a)most centered in the tube. The case of $R=6$ rapidly crosses the centerline (approximately $\times / D=$ 1.2 , where the jet enters at $x / D=1.00)$, and continues downstream predominantly in the upper half of the test section.
The swirl flow bubble pictures, shown in parts (b) and (c) of Figures 7, 8, and 9, illustrate the helical path of the jet and the strength of the precessing vortex core (PVC). In the case of $\phi=70$ degrees swirl, sufficient negative axial velocity occurs in the PVC to carry the bubbles upstream to the swirler face, regardless of the R-value. For the cases of moderate swirl, $\phi=45$ degrees, there is a noticeable difference in the jet-to-crossflow interaction. The case of $R$ $=2$ appears to mix broadly with the centrallylocated PVC, indicated by the wide jet outline and broad PVC. The cases of $R=4$ and 6 exhibit less immediate mixing with the precessing vortex core, tending to disturb its presence as the laterallyinjected jet penetrates across the central part of the main, where the PVC would otherwise be. The $R$ - 6 case in particular seems to "wrap around" the central axis - a smearing of bubbles can be seen on the tube inside wall. For the case of strong swirl, $\phi=70$ degrees, there is very little difference in the flowfield between the different injection velocity cases. The cases of $R=4$ and 6 , however, can be seen to slightly deflect the swirl axis at approximately $x / D=2.00$ (jet enters at $x / D=1.00)$. The case of $R=6$ does seem to lower the upstream penetration of the PVC, as exhibited by a lack of bubbles.

\subsection{Sparks}

Figures 10,11 , and 12 present spark-gap flow visualization pictures for the same cases of $R=2$, 4, and 6, using the method described in Chapter 3. These particular photographs were taken with the electrodes positioned at $x / D=1.50$ where the jet enters at $x / D=1.00$.

In part (a) of these figures, the camera is positioned to the side of the facility and a vertical rx-plane is observed. In the swirl flow cases of parts (b) and (c), a second camera was simultaneously operated from a downstream location to illustrate the ro-plane behavior of the sparks. In these swirl cases, both photographs have been combined to form a common picture. The respective cases $R=2,4$, and 6 with no swirl exhibit the change in the flowfield from $x / D=1.50$ and continuing downstream. The case of $R=2$ shows how the flowfield is merely deflected upward by the entering jet. The lower part of the arcs apparentiy are deflected around the jet, away from the control plane, hence a true 3-D effect on the photograph. The case of $R=4$ shows flowfield acceleration above and around the jet, which has its centerline nearly corresponding with the centerline of the tube. The 'fold-over' just above the jet centerline probably corresponds to the downflow around the jet as the jet displaces the crossflow in the upper half of the test section. The case of $R=6$ shows less uniform behavior. The arcs do appear to define the upper bounds of the jet and the turbulent region behind the jet.

The swirl flows presented in parts (b) and (c) of Figures 10, 11, and 12 are actually two photographs taken simultaneously by separate cameras; the two negatives are combined to print a common picture. Again, the electrodes were placed at $x / D=1.50$, with the lateral jet entering at $x / D$ $=1.00$. A wire was placed in the centerline of the tube to prevent the spark from arcing to the tube walls and to help define the tube centerline. The end views exhibit a great deal of reflection off of 


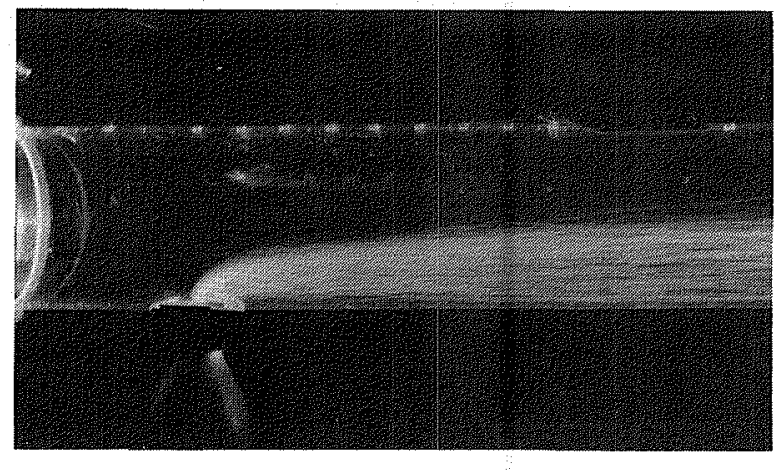

(a) $\phi=0 \mathrm{deg}$.

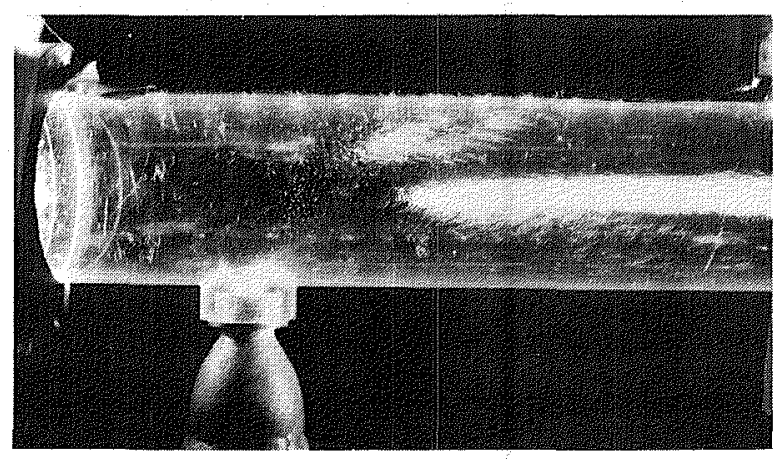

(b) $\phi=45 \mathrm{deg}$.
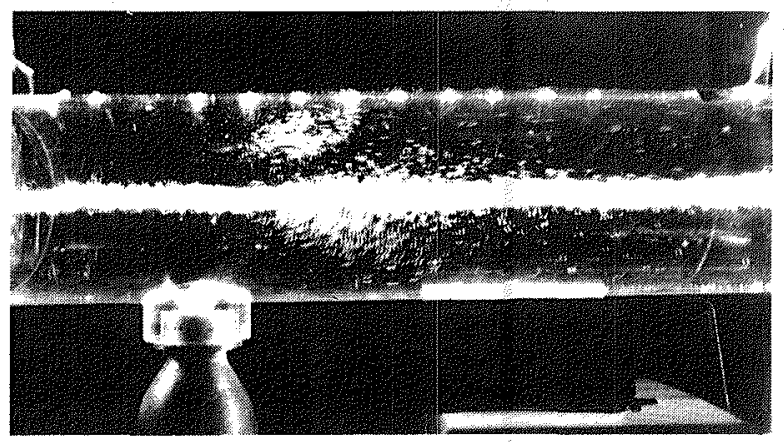

(c) $\phi=70 \mathrm{deg}$.

Fig. 7. Bubble Flow Visualization for Jet-toCrossflow Velocity Ratio $R=2.0$

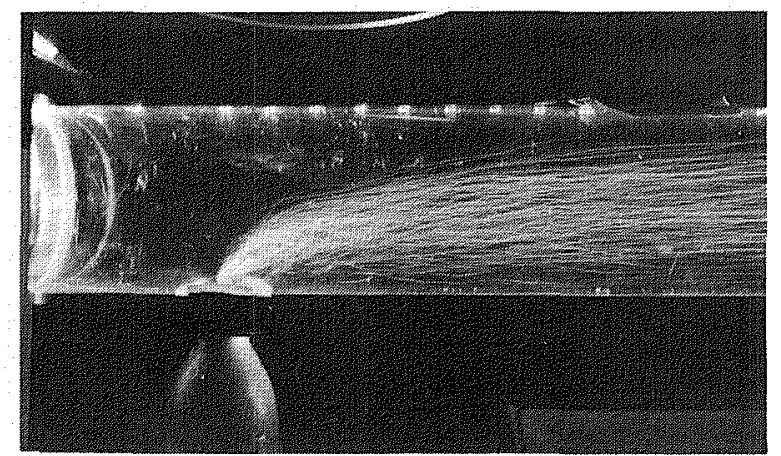

(a) $\phi=0 \mathrm{deg}$.

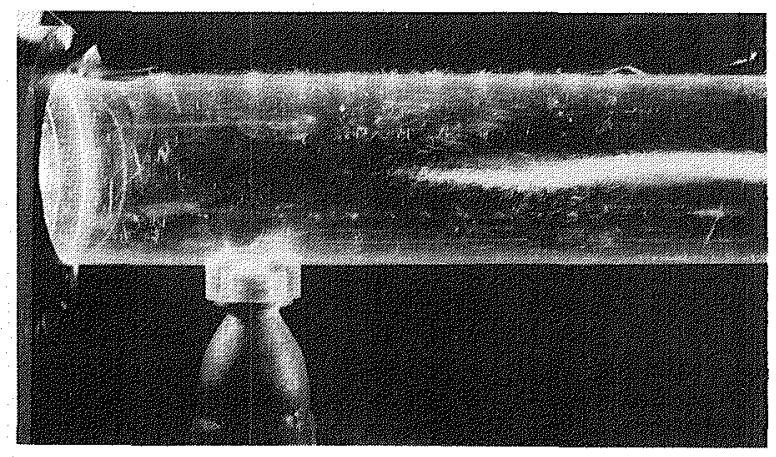

(b) $\phi=45 \mathrm{deg}$.

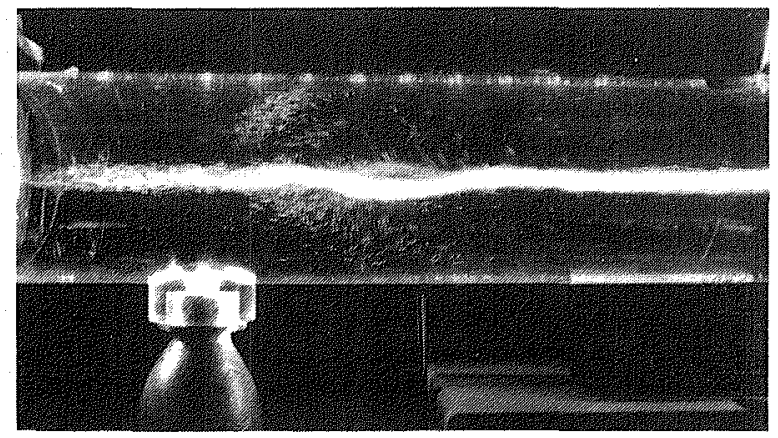

(c) $\phi=70 \mathrm{deg}$.

Fig. 8. Bubble Flow Visualization for Jet-toCrossflow Velocity Ratio $R=4.0$ 


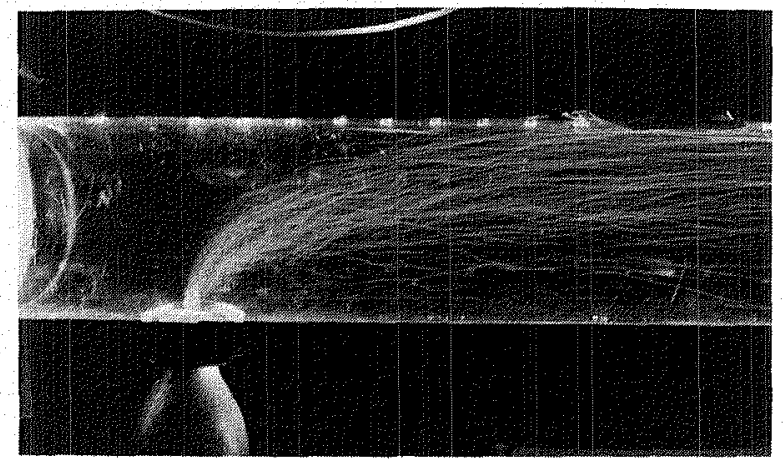

(a) $\phi=0 \mathrm{deg}$.

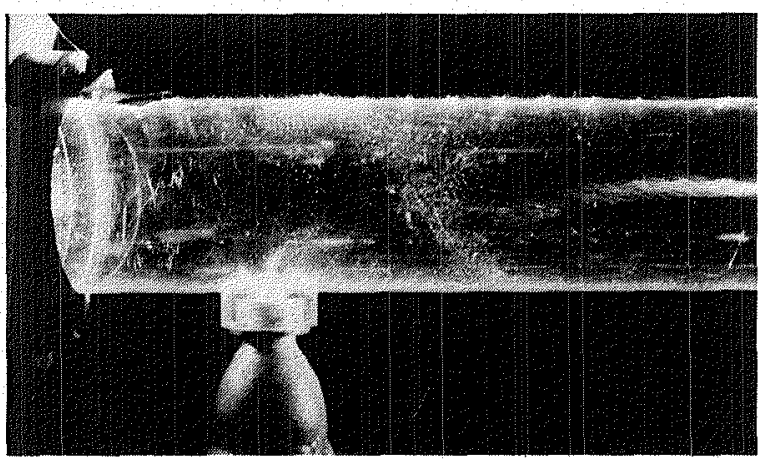

(b) $\phi=45 \mathrm{deg}$.

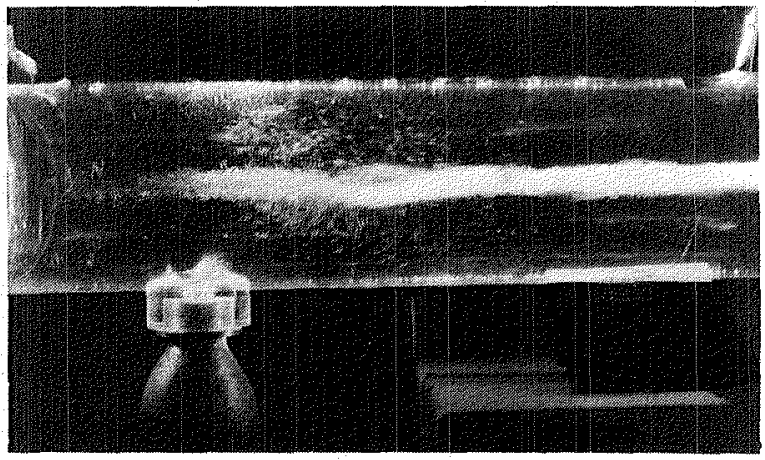

(c) $\phi=70 \mathrm{deg}$.

Fig. 9. Bubble Flow Visualization for Jet-toCrossflow Velocity Ratio $R=6.0$ the inside acrylic tube walls: only the central portion of the view is needed.

With moderate swirl ( $\phi=45$ degrees) the cases of $R=2$ and 4 have little effect on the swirl pattern shown with this technique. The case of $R=$ 6 , however, is seen to be deflected by the jet. The swirl strength seems to be enhanced in the lower part of the test section by the additional momentum of the deflected jet. With strong swirl ( $\phi=70$ degrees), the cases of $R=2$ and 4 seem to slightly inhibit the swirl strength, whereas the $R$ $=6$ case appears to have little effect except to organize the swirl pattern.

\section{CLOSURE}

Three flow visualization techniques were used to characterize the flowfield of a confined deflected jet, with and without swirl in the crossflow. Smoke flow visualization combined with short time-duration photographs exhibited the organized eddy structure for low values of the jetto-crossflow velocity ratio $R$. Values of $R=4$ and 6 exhibited large turbulent eddies. Both smoke and neutrally-buoyant helium-filled soap bubbles were used to trace the jet penetration and expansion. The long time-exposure bubble photographs demonstrated the mean flow paths of the jet volumes. The case of $R=2$ was found to affect only the lower half of the test section. The case of $R=4$ was found to cross the centerline and continue downstream very nearly centered in the flowfield. The case of $R=6$ was found to nearly impinge on the opposite wall of the test section and dramatically affect the flowfield in the upper half of the test section. A multi-spark flow visualization technique was used to manifest changes in the flowfield after injection of the lateral jet. The technique demonstrated the bulk effects of range of jet velocity ratios on the main flow, with a range of inlet swirl strengths in the main flow. Multi-spark photographs taken in the cases with swirl demonstrate the effect of lateral jet momentum on vortex core mixing and swirl strength. The flow visualization results agree well with the measurements obtained elsewhere with the six-orientation single hot-wire method.

\section{ACKNOHLEDGMENTS}

Gratitude is expressed to NASA Lewis Research Center for assistance via Grant No. NAG 3-159, technical monitor Dr. J. D. Holdeman. Help with the experiments came from several students, notably Bobby W. Evans, Steve S. Meador, Lawrence H. Ong, Valerie A. Smart and Mark A. Southard, to whom special thanks are offered.

\section{REFERENCES}

1. Lefebvre, A. H., "Gas Turbine Combustion." McGraw-Hill, New York, 1983.

2. Gupta, A. K., Lilley, D. G., and Syred, N. "Swirl Flows." Abacus Press, Tunbridge Wells, England, 1984.

3. Gupta, A. K. and Lilley, D. G., "Flowfield Modeling and Diagnostics." Abacus Press, Tunbridge Wells, England, 1985.

4. Lilley, D. G., "Swirling Flows in Typical Combustor Geometries," Paper AIAA-85-0184, 


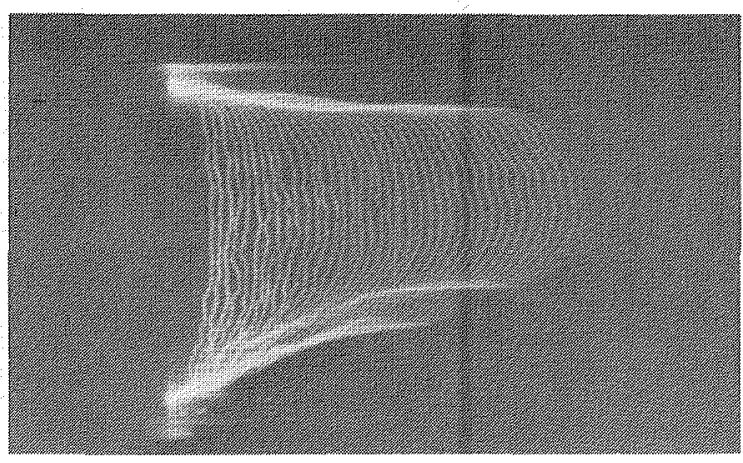

(a) $\phi=0 \mathrm{deg}$.

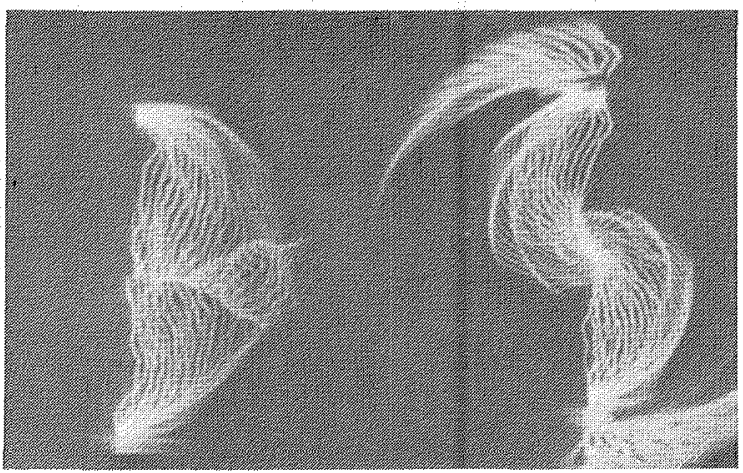

(b) $\phi=45 \mathrm{deg}$.

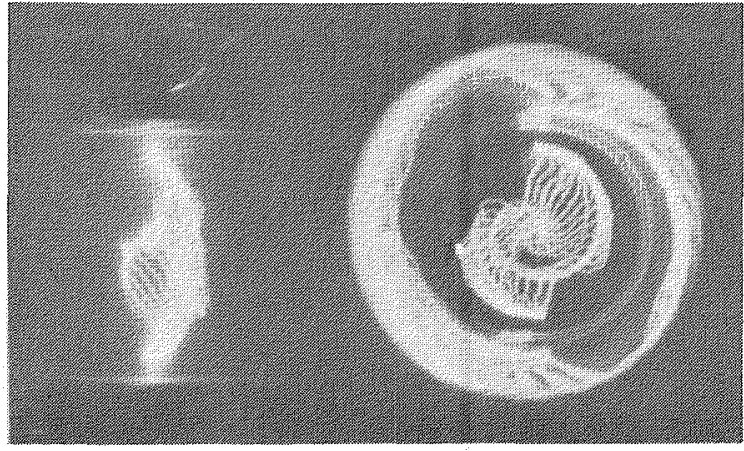

(c) $\phi=70 \mathrm{deg}$.

Fig. 10. Spark-Gap Flow Visualization for Jet-toCrossflow Velocity Ratio $=2.0$

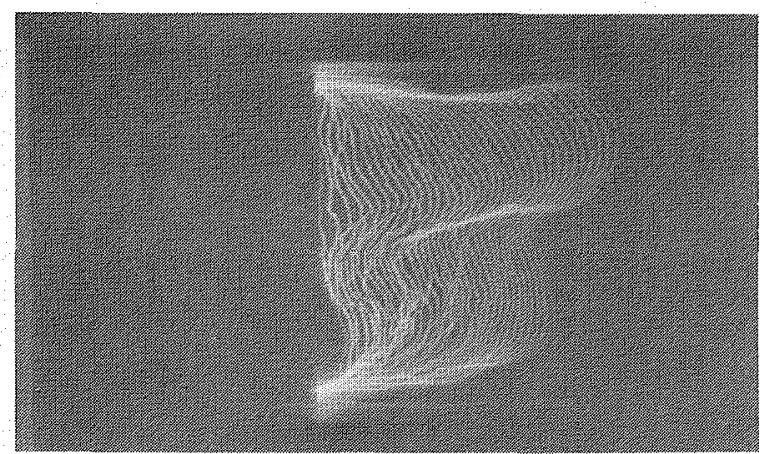

(a) $\phi=0$ deg.

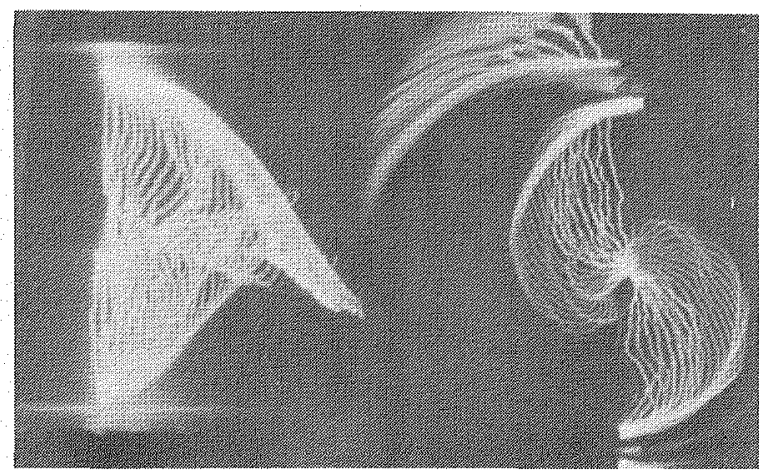

(b) $\phi=45 \mathrm{deg}$.

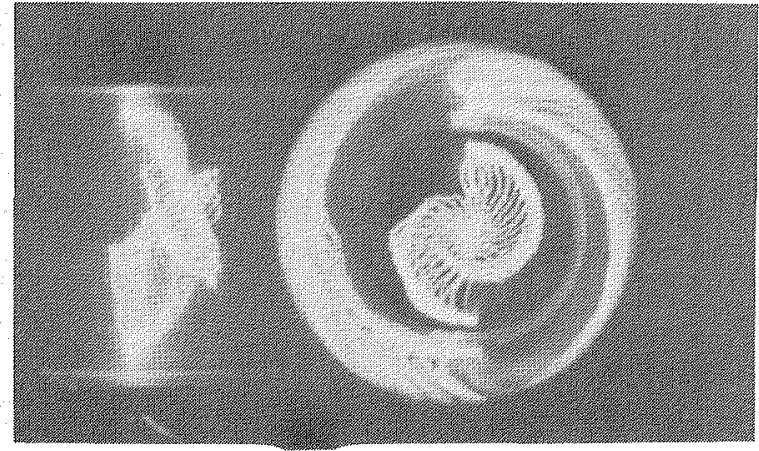

(c) $\phi=70 \mathrm{deg}$.

Fig. 11. Spark-Gap Flow Visualization for Jet-toCrossflow Velocity Ratio $=4.0$ 


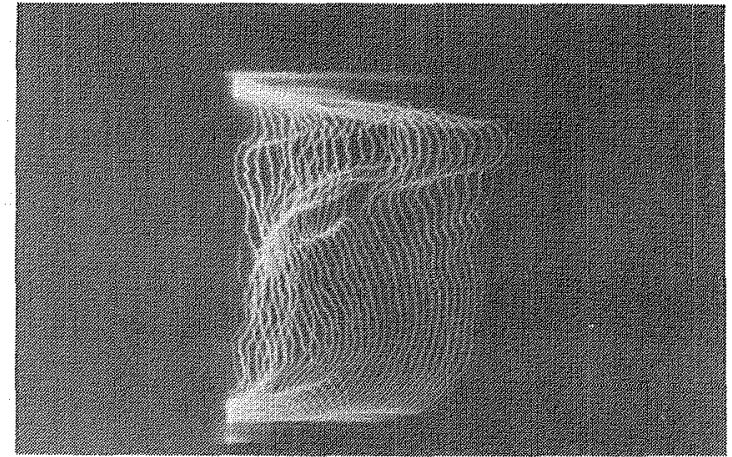

(a) $\phi=0$ deg.

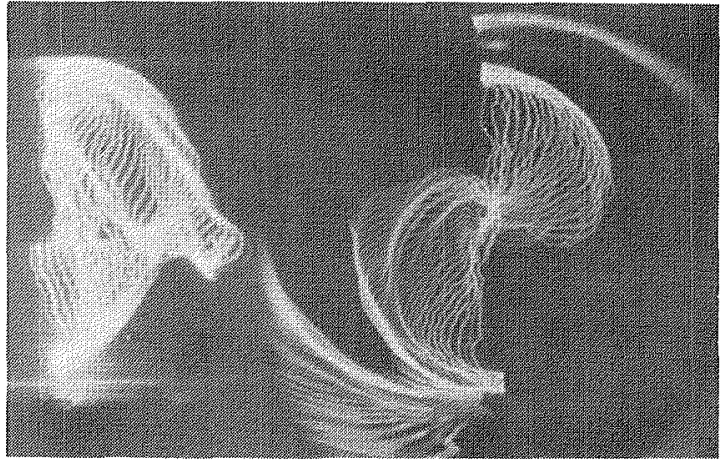

(b) $\phi=45 \mathrm{deg}$.

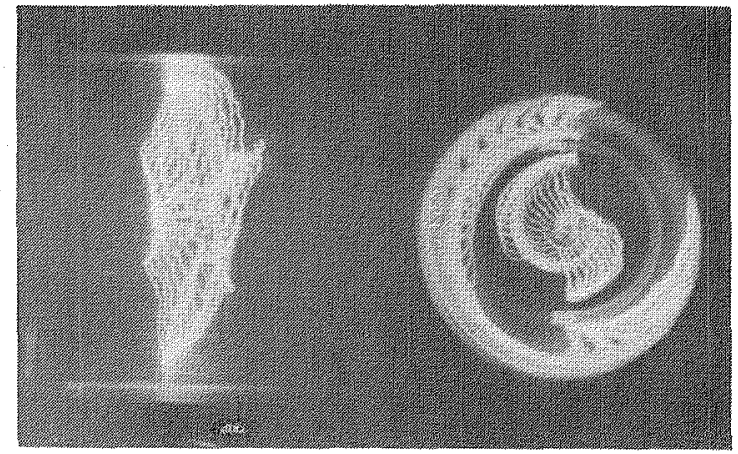

(c) $\phi=70 \mathrm{deg}$.

Fig. 12. Spark-Gap Flow Visualization for Jet-toCrossflow Velocity Ratio $=6.0$
Reno, Nevada, Jan. 14-17, 1985.

5. Ferrell, G. B., Abujelala, M. T., Busnaina, A. A., Lilley, D. G., "Lateral Jet Injection into Typical Combustor Flowfields", AIAA Paper 84-0374, Reno, Nevada, Jan. 9-12, 1984.

6. Ferrell, G. B., "Deflected Jet Experiments in a Turbulent Combustor Flowfield." Ph.D. Thesis, 0klahoma State University, Stillwater, Okla., Dec. 1984.

7. Chassaing, P., George, J., Claria, A., and Sananes, F., "Physical Characteristics of Subsonic Jets in a Cross-stream", Journal of Fluid Mechanics, Vol. 62, 1974, p. 4164.

8. Rathyeber, D. E., and Becker, H. A., "Mixiny Between a Round Jet and a Transverse Pipe Flow", Proceedings of lst Symposium on Turbulent Flows, Penn State University, 1977, pp. 10.17-10.26.

9. Crabb, D., Durao, D. F. G., and Whitelaw, J. W., "A Round Jet Normal to a Cross-Flow", ASME Trans., Vol. 103, 1981, pp. 142-153.

10. Holdeman, J. D., Srinivasan, R., and Berenfeld, A., "Experiments in Dilution jet Mixing", Paper AIAA-83-1201, Seattle, Wash., June 27-29, 1983, AIAA Journal, Vol. 22, No. 10, 1984, pp. 1436-1443.

11. Schetz, J. A., "Injection and Mixing in Turbulent Flow", Prog. in Astro, and Aero., AIAA, Vol. 68, 1980.

12. Merzkirch, W., "Flow Visualization", Academic Press, New York and London, 1974, pp. 1916. 620.1064 - M577f.

13. Rhode, D. L., Lilley, D. G., and McLaughlin, D. K., "Mean Flowfields in Axisymmetric Combustor Geometries with Swirl", AIAA Journal, Vol, 21, No. 4, April 1983, pp. 593-600.

14. Meador, S. S. and Southard, M. A., "MultiSpark Visualization of Typical Combustor Flowfields", Senior Project Report, School of Mechanical and Aerospace Engineering, Oklahoma State University, Stilliwater, Okla., Dec. 1984.

15. Nakayama, Y., Okitsu, S., Aoki, K., and Ohta, H., "Flow Direction Detectable Spark Method", The International Symposium on Flow Visualization, Tokyo, Japan, Oct. 1977, pp. 163-168.

16. Nakayama, Y., Aoki, K., Ohta, H., and Wakatsuki, M., "Spark Method Visualization of the Swirling Flow Mixing Process", Proc. 3rd Int. Symp. on Flow Visualization, Ann Arbor, Michigan, Sept. 1983, pp. 481-485.

17. Jorski, M. L., Knipe, R. L., Piccolo, D. B., and Smart, V. A., "Lateral Jet injection Into Swirling Crossflow of a RoundSectioned Test Section", Senior Project Report, School of Mechanical and Aerospace Engineering, Oklahoma State University, 
Stillwater, Okla., July, 1984.

18. Bomelburg, H. J., Herzog, J. R., and Weske, J. R., "The Electric Spark Method for Quantitative Measurements in Flowing Gases", Z. Flugwiss. Vol. 7, 1959, pp. 322329.

19. Kyser, J. B., "Tracer-Spark Technique for Velocity Mapping of Hypersonic Flow Fields", AIAA Journal Vol. 2, 1964, pp. 393-394.

20. Lahaye, C., Leger, E. G., and Lemay, A., "Wake Velocity Measurements Using a Sequence of Sparks", AIAA Journal Vol. 5, 1967, pp. 2274-2776.

21. Morel, T., "Comprehensive Design of Axisymmetric Wind Tunnel Contractions", ASME Paper 75-FE-17, Minneapolis, MN, May $5-7,1975$.

22. Sander, G. F., and Lilley, D. G., "The Performance of an Annular Vane Swirler", Paper AIAA-83-1326, Seattle, Wash., June 27-29, 1983. 
APPENDIX C

TURBULENCE MEASUREMENTS OF LATERAL JET INJECTION

INTO CONFINED TUBULAR CROSSFLOW

AIAA $-85-1102$ 


\title{
INTO CONFINED TUBULAR CROSSFLOW
}

\author{
G. B. Ferre11* \\ Texas Tech University, Lubbock, Texas \\ and \\ D. G. Lilley** \\ Oklahoma State University, Stillwater, Oklahoma
}

\section{ABSTRACT}

\begin{abstract}
Experiments have been conducted to characterize the time-mean and turbulent flowfield of a deflected turbulent jet in a confining cylindrical crossflow. Jet-to-crossflow velocity ratios of 2,4 , and 6 were investigated, with no swirl in the crossflow. A six-position single hotwire technique was used to measure the velocities and all turbulent normal and shear stresses. Measurements confirmed that the deflected jet is symmetrical about the vertical plane passing through the crossflow axis, and the jet penetration was found to be reduced from that of comparable velocity-ratio infinite crossflow cases.
\end{abstract}

\section{NOMENCLATURE}

\begin{tabular}{|c|c|}
\hline $\begin{array}{l}A, B, C \\
A_{C}\end{array}$ & $\begin{array}{l}\text { calibration constants } \\
\text { cross-sectional area of } \\
\text { crossflow }\end{array}$ \\
\hline${ }_{D}^{A}$ & $\begin{array}{l}\text { cross-sectional area of jet } \\
\text { test section diameter }\end{array}$ \\
\hline d & crossflow inlet nozzle diameter \\
\hline$d_{\mathrm{E}}$ & $\begin{array}{l}\text { lateral jet inlet diameter } \\
\text { not-wire voltage }\end{array}$ \\
\hline $\mathrm{G}$ & pitch factor \\
\hline $\mathrm{k}$ & yaw factor \\
\hline $\begin{array}{l}\text { R } \\
\text { RAM }\end{array}$ & $\begin{array}{l}\text { Jet-to-crossflow velocity ratio } \\
\text { random access memory }\end{array}$ \\
\hline$\frac{\mathrm{Re}}{\mathrm{V}}=(\mathrm{u}, \mathrm{v}, \mathrm{w})$ & $\begin{array}{l}\text { Reynolds number } \\
\text { time-mean velocity in facility } \\
\text { coordinates ( } x^{-}, \mathrm{r}^{-} \\
\theta \text {-directions) }\end{array}$ \\
\hline$x, r, \theta$ & $\begin{array}{l}\text { axlal, radial, azlmuthal } \\
\text { coordinates }\end{array}$ \\
\hline $\mathbf{Y}$ & vertical distance above jet \\
\hline $\mathrm{Z}$ & $\begin{array}{l}\text { effective cooling velocity } \\
\text { acting on a wire }\end{array}$ \\
\hline$\gamma_{z_{1}} z_{j}$ & $\begin{array}{l}\text { correlation coefficient } \\
\text { (estimated) between cooling } \\
\text { velocities }\end{array}$ \\
\hline$\phi$ & $\begin{array}{l}\text { swirl vane angle with respect } t \\
\text { facility axis }\end{array}$ \\
\hline$\theta$ & traverse azimuthal angle \\
\hline
\end{tabular}

Subscripts

$\begin{array}{ll}1, j & \text { summation indices } \\ 0 & \text { value at inlet to flowf leld } \\ \text { rms } & \text { root-mean-squared }\end{array}$

* Assistant Professor, Department of Mechanical Engineering, Member AIAA.

* Professor, School of Mechanical and Aerospace Engineering, Associate Fellow AIAA.
Superseripts
()
()
time-mean average
fluctuating component
relative to probe coordinates

\section{INTRODUCTION}

\subsection{The Problem}

Deflected turbulent jets in gas turbine combustion chambers provide a compllcated example of fully 3-D flowfields. In addition, highintensity combustion takes place. Whereas most fuel burning takes place in the primary zone, the secondary zone is where combustion is almost completed, and the dilution zone is where further temperature reduction and uniformity is achleved. In both can and annular combustors, lateral jets of cooler air through round holes penetrate the flowfleld. Some of these jets amalgamate with the swirl-induced central recirculation region of the primary zone and provide sufficlent additional air for stolchiometric conditions. Other lateral jets provide additional atr to help complete combustion in the secondary zone and to cool and evenly mix the products in the dllution zone in preparation for the introduction of the flow to the turbine. Similar problems arise in ramjet combustors. clearly the turbulent reacting flowfleld is fully three-dimensional; the combustion deslgner has a formidable problem in aerothermochemlstry, and the research and development task is to provide a route which leads to the accomplishment of deslgn objectives more quickly and less expensively than current practice permits. Recent textbooks ${ }^{1-4}$ extensively review progress and problems in this area.

\subsection{Objectives}

The present study concentrates on a single lateral jet being injected normally into nonswirling crossflow - later work, currently in progress, will remove these restrictions. The alm is to provide a suitable data base for characterizing the flowfleld, permfting modeling advances and turbulence model developments. Earlier studies described elsewhere ${ }^{5,8}$ focused on the gross flowfield via fully 3-D predictions flow visualization studies, including the use of neutrally-buoyant hellum-fllled soap bubbles, smoke, and multi-sparks. The flow visualization study was used to characterize one deflected jet in crossflow without swirl (swirler removed) and with swirler angles of 45 and 70 degrees.

The present paper describes the application of a hot-wire measurement technique. A computerized

This paper is declared a work of the U.S. Government and therefore is in the public domain. 
data acquisition and reduction technique was developed and implemented. Because of the large number of data point locations and random nature of the fluctuating velocities, a high-speed analog-todigital data acquisition system with a computercontrolled probe drive was designed and constructed. A six-orientation single hot-wire technique was used to map fully the time-mean and turbulent velocity flowfleld. The data acquisition and probe drive system was used to manlpulate the probe and hot-wire voltages. Jet to cross-flow velocity ratios of 2,4 , and 6 were used with no swirl in the crossflow.

\subsection{Outline of the Paper}

Section 2 provides background to the present study. A description of the experimental facility and measurement equipment is given in Section 3; the data acquisition and probe drive system (designed speciflcally for this investigation) is fully discussed. The single-wire multi-orientation measurement technique is described in section 4 . Results are presented in Section 5, and discussed in the light of previous flow visualization evidence. Section 6 draws conclusions from the study.

\section{BACKGROUND}

2.1 Review of Previous studies in Deflected Turbulent Jets

A number of experimental investigations of the jet in a cross-flow have been reported in the literature. As early as 1948, Callaghan and Ruggeri ${ }^{7}$ examined a heated $200^{\circ} \mathrm{C}$ alr jet directed normal to the wind-tunnel flow. The heated jet exhausted into the confined tunnel through a sharpedged orifice. Jet relocities from approximately two to seven times the cross-stream velocity were investigated for penetration depth and mixing. Thermocouple and pitot tube rakes were utilized well downstream of the jet inlet to record penetration depth and mixture temperatures. Ruggeri, Callaghan, and Bowden extended this work in 1950 to include square and elliptical orifices.

In 1952 , Rugger $1^{\circ}$ extended his contribution to include various angles ( $30,45,60$, and 90 degrees) of injection. The angles required that a tube be utillzed to inject the jet, as opposed to a sharpedged oriflce. Ruggeri used Schlleren flow visualization to confirm his measurements and commented on the wall effects of the wind tunnel.

Jordinson, ${ }^{10} 1958$, was the first to determine experimentally the trajectory of the jet crosssection and defined the jet axis as the line connecting the points of maximum velocity. He also demonstrated that the cross-section of an initially cylindrical jet is distorted into a 'horseshoe' shape by the cross-stream shearing action.

Keffer and Baines, 1", 1963, contributed some very carefully measured velocity data. More importantly, they studied the turbulent structure in the deflected jet and showed that similarity for a reasonably small range of velocity ratios ( 4,6 , and 8 ) could be shown. Their work was the first use of a jet-oriented coordinate system, relating the jet centerline to that of a free jet. Pratt and Bainesiz refined this work to account for scatter in the previous data, and also demonstrated that the proflle of the jet is conserved as a mixture between circular cross-section of random eddies and a pair of line vortices with small turbulences. Pratt and Keffer's continued this investigation for various injection angles $(60,75$, 90, 115, and 135 degrees) to the main flow. Finding differences between their jet trajectories and that of Keffer and Baines"', they recognized the importance of the jet inlet velocity profile. In fact, their experiments used long tubular inlets whereas Keffer et al used a simple orifice inlet in the earlier study"'.

Some of the first work to examine multiple deflected jets is represented by Norgren and Humenik ${ }^{14}$, 1968, intended to aid in the design of short-length combustors. As with the ploneering work of Callaghan, ${ }^{7}$ they restricted their investigation to penetration depth and degree of mixing for heated jets. It should be noted that this work was one of the first basic research studies into turbine inlet temperature profiles.

In 1973, Campbell and Schetz ${ }^{25}$ developed one of the first analytical models of a deflected jet and also verifled this integral method with experiments. Their model was successful in predicting flow velocities and trajectorles in buoyant, heated jets.

Kamotoni and Greber ${ }^{16}$ were probably the first to study multiple jet injection into a confined cross-flow. The single row of jets was directed toward the opposite wall of a rectangular crosssection wind tunnel. Most interesting of their conclusions was that the jets were only mildly affected by the opposite wall, unless the jet directly impinged upon that wall. Holdeman and Walker ${ }^{17}$ extended this work and others to develop an empirical model predicting downstream temperature profiles.

Chassaing, et al, ${ }^{18}$ in 1974 , contributed works comparing several zones of simliarity of the jet in cross-flow problem.

The most detalled work thus far for a single deflected jet has been that of Crabb, Durao and Whitelaw.' ${ }^{19}$ Utilizing laser-doppler anemometry in the region of the jet, their measurements extensively quantify the velocity field with its assoclated turbulence and vortex pairs. The only detraction is that the jet to cross-flow velocity ratios of their measurements are quite low -- 1.15 and 2.30. Most investigators ${ }^{\text {- } 14}$ have been in the range of 4-10 times the cross-stream velocity as this approaches the dilution jet case more closely. The ratio of 1.15 would fall slightly above the region of $\mathrm{film}$ cooling. A tubular inlet was used to inject the jet perpendicularly into a large wind tunnel.

The work of Rathgeber and Becker20 is representative of few Investigations dealing with jet injection into a cylindrical cross-section flow. They investigated relatively small diameter jets as compared to the cross-flow dlameter (crossflow to jet dlameter ratios between 17.2 and 50 ). These measurements quantify mixture and trajectory data, but do not deal with turbulence detalls.

Holdeman and colleaguesi-24 describe NASA studies of multijet injection into rectangularsectioned crossflow, including extensive results 
and empirical characterization. On the other hand, direct numerical solution ${ }^{2-28}$ has inspired the need for further experimental evidence and full documentation about inlet conditions and turbulence field data. These and other studies are extensively reviewed elsewhere. 29,30

\subsection{Past Work at Oklahoma State University}

Recently, as summarized by Lilley," experimental and theoretical research has been completed on 2-D axisymmetric geometrles under low speed, nonreacting, turbulent swirling flow conditions, in the absence of any lateral jets. The flow enters the test section and proceeds into a larger chamber (the expansion $D / d=2$ ) via a sudden or gradual expansion (side-wall angle $\alpha=90$ and 45 degrees). A weak or strong nozzle may be positioned downstream to form a contraction exit to the test section. Inlet swirl vanes are adjustable to a a variety of vane angles with values of $\phi=0,38,45,60$ and 70 degrees being emphasized. The objective was to determine the effect of these parameters on 1sothermal flowfleld patterns, time-mean velocities and turbulence quantities, and to establish an improved simulation in the form of a computer prediction code equipped with a suitable turbulence model.

In conjunction with these research objectives, several experimental techniques have been developed including:

1. Flow visualization, achieved via still and movie photography of neutrally-buoyant hellum-filled soap bubbles, smoke produced by an injector and multisparks across the flowfleld.

2. Time-mean velocities have been measured with a five-hole pitot probe for a complete range of swirl strengths. 3. Turbulence measurements have been
completed on swirling (up to $\phi=70$ degrees) as well as nonswirling flows using a six-orlentation single-wire hotwire technique, enabling all Reynolds stress components to be deduced.

4. An advanced computer code has been developed and improved to predict corresponding confined swirling flows to those studied experimentally.

5. Tentative predictions have now been supplemented by predictions made from realistic inlet conditions for a complete range of swirl strengths with downstream nozzle effects.

Recent changes to the experimental facility to enable one and/or two laterally-injected jets to be Included are described in Refs. 5, 6, 29 and 30. Additionally, tentative 3-D predictions for corresponding flowfields are given by Busnaina32,33.

\section{EXPERIMENTAL FACILITY}

\subsection{Hind Tunnel}

The test facility, dominated by the wind tunnel, is shown schematically in Figure 1. Air is

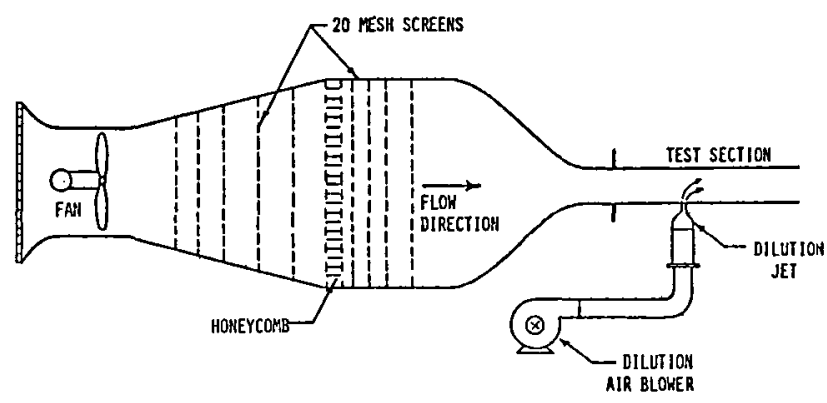

Fig. 1. Schematic of Experimental Facility

induced to flow through a large foam inlet fliter by an axial fan. The fan is connected by way of belts and pulleys to a seven-horsepower U.S. Motor varidrive. The varidrive permits the fan speed to be adjusted from 500 to 2850 RPM. The air is then forced into an expanding area section, where multiple 20-mesh screens and a section of straws are employed to straighten the flow and significantly lower the turbulence intensity.

Before introduction to the test section, the air flows through an axisymmetric nozzle with an area reduction ratio of 25 . The nozzle was bullt with a matching cublc radial proflle, according to the method described by Morel ${ }^{34}$. The objective of this nozzle design is to produce a low turbulence level unlform velocity proflle before introduction to the test section, with minimum adverse pressure gradient in the boundary layer. The exit throat diameter of the wind tunnel is approximately $15 \mathrm{~cm}$.

At the throat of the tunnel and before introduction to the test section, a varlable-angle swirler may be fitted. The swirler consists of ten flat blades (with pitch-to-chord ratio 0.68 ) which may be Individually adjusted to any angle from 0 to 70 degrees. Detalled information and measurements related to the swirler performance and swirler exit velocity profiles on this facllity are given in Ref. 31 .

\subsection{Test Section and D1lution Jet}

The test section consists of a clear acrylic tube approximately $90 \mathrm{~cm}$ in length attached to the wind tunnel throat. Standard commerclal acrylic tube is used with $15.24 \mathrm{~cm}(6.0 \mathrm{in.})$ outside diameter, $0.318 \mathrm{~cm}(0.125 \mathrm{in}$.) wall thickness. The inside diameter $1 \mathrm{~s} 14.61 \mathrm{~cm}$ with a measured varlation of $+0.05 \mathrm{~cm}$. To adapt the test section to the wind tunnel throat (inslde dlameter $15 \mathrm{~cm}$ ), an adaptor section was machined to provide a smooth transition from wind tunnel throat to test section. Two test section tubes were constructed. Both test sections have the dilution jet inlet located at $x / D=1.00$ where $x$ is measured from the tube inlet. The first tube has a series of probe access holes located at $x / D=1.00,1.25$, and 1.50 and at all six azimuthal locations 270, $300,330,0,30$ and 60 degrees as shown in F1gure 2. The second tube allows probe access to locations downstream of $x / D=1.50$ (for example $x / D$ $=1.75,2.00,2.50,3.00)$ and at any azimuthal angle. This is accomplished via a tube rotation 

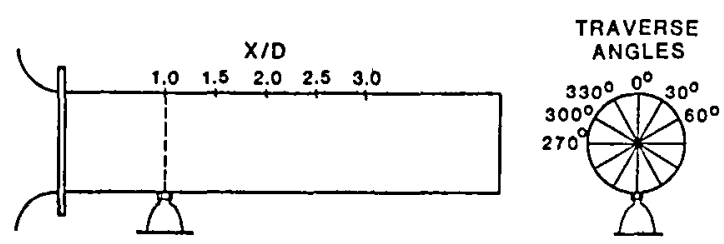

Fig. 2. Test Section Geometry
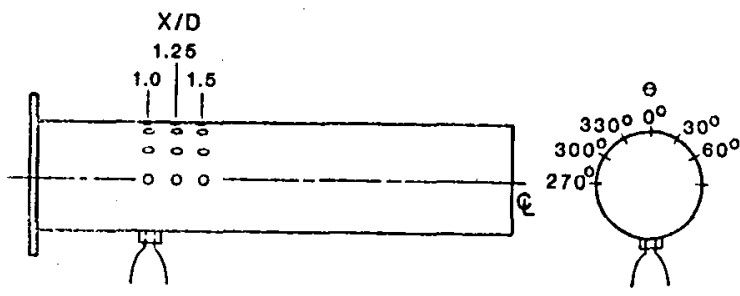

A. FIXED TUBE

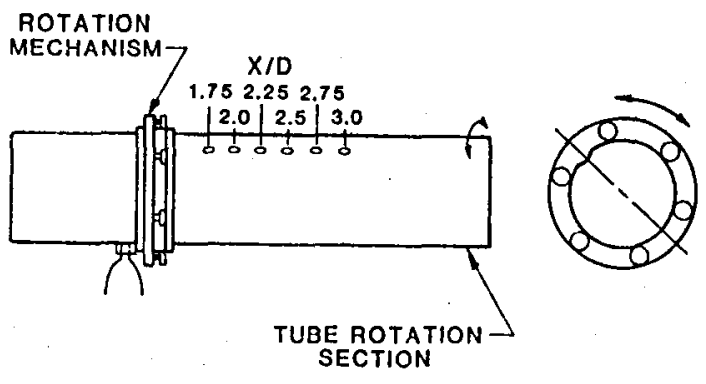

B. ROTATION TUBE

F1g. 3. Test Sections

section, constructed from machined aluminum rings, acrylic, and ball bearings as seen in Figure 3 .

Laboratory compressed air at 6 to 7 atmospheres gauge pressure is used to supply the dilution jet. For stability, the supply alr lines are large and are routed through two line regulators with an intermedlate tank (volume approximately $\quad 0.006 \mathrm{~m}^{3}$ ) to dampen line oscillations. The second regulator was used to meter the flow rate. After the second regulator, the air was routed through a Fisher and Porter model 10A1735A rotometer for monltoring of the volume flow before introduction to the dilution jet. The dilution jet assembly consists of a stagnation chamber, flow stralghtening section, and the jet nozzle. The stagnation chamber was constructed from $15 \mathrm{~cm}$ inside diameter aluminum pipe and filled with plastlc household scrub pads to evenly distribute the internal flow. A hemlspherically-shaped screen and convergent transition smooth the flow into the flow stralghtening section. Here the alr flows through four brass screens for turbulence reduction. The nozzle was deslgned according to the method suggested by Morel ${ }^{34}$ and is constructed of fiberglass. The nozzle diameter is one-tenth of the test-section diameter, giving a cross-flow to jet area ratio $\left(A_{c} / A_{f}\right)=100$. Construction of the nozzle, a multi-step process, consisted of: constructing a two-dimensional contour on a numerically-controlled milling machine from a computer-generated proflle, using the contour and a hydraulic follower on a lathe to produce an axisymmetric male mold, and forming the fiberglass nozzle around the male mold, with aluminum flanges formed in.

Once assembled, the dilution jet was attached to the alr line coming from the rotometer and the nozzle was pressed into a speclal acrylic adaptor which is permanently attached to the test sectlon.

\subsection{Hot-Hire Instrumentation}

The sensing transducer used in this study is a normal hot-wire probe, DISA type 55P01. This probe has two prongs set $3 \mathrm{~mm}$ apart with a $5 \mu \mathrm{m}$ diameter tungsten wire between them. The exposed, effective length of the wire is approximately $1 \mathrm{~mm}$, since the ends have been gold plated to strengthen the wire and reduce end effects. The probe support was a standard DISA 55H21 stralght mounting tube. The anemoreter used was a DISA type 55M01, constanttemperature standard bridge. The hot-wire voltage was measured with the computer-controlled data acquisition system, discussed in Section 3.5 .

\subsection{Calibration Equipment}

A small axisymmetric free jet was employed to calibrate the not-wire. The calibration jet facility consists of a contoured nozzle similar in shape to the dilution jet and wind tunnel contraction. A settling chamber and turbulence management section consisting of packed straws is just upstream of the nozzle. The nozzle exit dlameter is $34 \mathrm{~mm}$. Using the standard laboratory air supply, the calibration jet is capable of producing Reynolds numbers up to $6 \times 10^{5}$ (based on throat dlameter). The alr supply is thermally stablilzed by virtue of long indoor lines and is within $+0.5^{\circ} \mathrm{C}$ of the facility temperature. The air is metered by means of a diaphragm valve and a Fisher and Porter model 10A1735A rotometer. The jet was callbrated using a pitot probe 1.0 nozzle diameters downstream of the exit plane. The temperature of the jet and the pressure before the rotometer were monitored during each callbration to account for minor varlations from the initial callbration velocitles. The hot-wire was placed in the potential core of the jet during callbration. Utilizing a rotary table and two not-wire support tubes (DISA 55H151, DISA 55H153), the hot-wire was callorated in the $u, v$, and $w$ directions.

\subsection{Data Acquisition and Probe Drive System}

The probe drive, shown mounted on the test section in Figure 4, was specifically designed for these investigations. The probe is positioned in the flowfield by two stepper-motors, one motor for rotation and the other for translation. The probe is held within the square slider by a cylindrical holder with 0 -rings to grasp the internal walls at any desired location. Both stepper motors step 200 times per motor shaft revolution. The rotation motor is geared down $3: 1$ so that 600 motor steps correspond to one probe revolution. For example, a probe rotation of 30 degrees requires exactly 50 


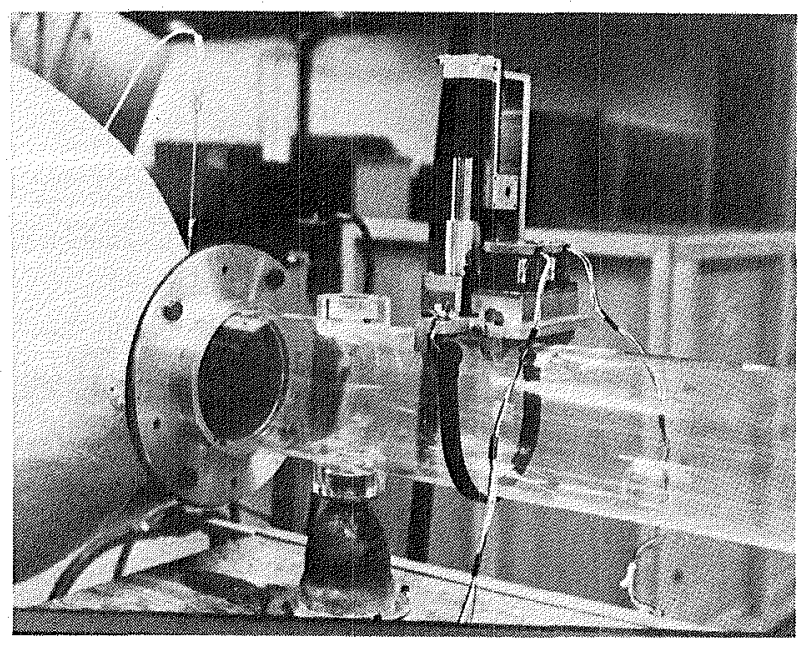

Fig. 4. Probe Drive Mounted on Test Section

steps. The software does not allow the probe to rotate in either direction more than one revolution to prevent cable twisting and colling.

The translation motor is geared down $3: 1$ to a lead screw, which has a linear gear ratio of 2.24 revolutions per $\mathrm{cm}$ (equivalent to 5.69 revs. per inch) translation. The effective step count for translation is therefore 1344 steps per om (3414 steps per inch). With gear lash considered, the translation resolution is less than $0.03 \mathrm{~mm}$ and the rotation resolution is less than 0.5 degrees. The mass of the probe drive is approximately $3.9 \mathrm{~kg}$ (8.5 $\mathrm{lbm})$ and is fastened to the test section with a large rubber binding strap. Reference 35 provides a detailed description of the probe drive and design philosophy.

An Apple II computer was used to sample the hot-wire voltage and control the probe-drive stepper motors. A Burr-Brown SDM853 12-bit A/D converter was utilized to convert the $0-10$ volt not-wire signals to 12-bit digital words. The Apple II controls, via assembly code, the sample times and accepts the data as two 8-bit words directly in RAM. Further machine codes are used to reassemble the samples, take an average and standard deviation and store the results. BASIC code is used to reassemble these 8 -bit words into decimal equivalent of 12-bit resolution. The system resolution is 2.44 millivolts. The data acquisition sample rate was fixed at 1000 samples per second for 5 seconds. A higher sampling rate (up to $30 \mathrm{kHz}$ ) could be utilized with more memory available. Reference 36 provides a detailed explanation of the data acquisition system and the assembly codes used.

\section{MEASUREMENT TECHNIQUES AND ANALYSIS}

In a turbulent, three-dimensional flowfield the main flow direction may be unknown and conventional hot-wire or 2-D Laser-Doppler techniques fail to supply sufficient velocity vector information. To measure the three velocity components and their corresponding fluctuations, a three-wire hot-wire probe is often used. Few 3-D Laser-Doppler systems are in use and are not cost- effective. The three-wire probe technique has several drawbacks. Three anemometers are required. A multiple-orientation probe drive may be needed to align the probe with the mean flow direction. Because of the physical separation of the wires, spatial resolution of the probe is poor.

Multi-orientation of a single hot-wire is a novel way to measure the three components of a velocity vector and their fluctuating components. King ${ }^{3}$ modified a technique developed by Dvorak and Syred. ${ }^{38}$ This method calls for a normal hot wire to be oriented through six different positions, each orientation separated by $30 \mathrm{deg}$ from the adjacent one. Orientation 1 is normal to the facility centerline, orientation 2 is rotated 30 deg from this, etc. Mean and rms voltages are measured at each orientation. The data reduction is performed using certain assumptions regarding the statistical nature of turbulence, making it possible to solve for three time-mean velocities, the three turbulent normal stresses, and the three turbulent shear stresses.

The six-orientation hot-wire technique requires a single, straight, hot wire to be calibrated for three different flow directions in order to determine the directional sensitivity of the probe. In the following relationships, tildes $(-)$ signify components of the instantaneous velocity vector in coordinates on the probe. Each of the three calibration curves is obtained with zero velocity in the other two directions. The hot wire is most efficlently cooled when the flow is in the direction of the $\vec{u}$ component (which is normal to both the wire and the supports). The wire is most inefficiently cooled when the flow is in the direction of the $w$ component (which is parallel to the wire). Each of the calibration curves follows a second-order, least-square fit of the form

$$
E_{i}^{2}=A_{i}+B_{i} \tilde{u}_{i}^{1 / 2}+C_{i} \tilde{u}_{i}
$$

which is an extension of the familiar King's law. In this equation, $A_{i}, B_{i}$, and $C_{i}$ are calibration constants and $u_{i}$ can take on a value of $\tilde{u}, \tilde{v}$, and $\tilde{w}$ for the three calibration curves, respectively.

When the wire is placed in a three-dimensional flowfield, the effective cooling velocity experienced by the hot wire is

$$
z^{2}=\tilde{v}^{2}+G^{2} \tilde{u}^{2}+K^{2} \tilde{w}^{2}
$$

where $G$ and $K$ are the pitch and yaw factors defined by Jorgensen ${ }^{39}$ and deduced from the calibration curves. Hence, equations for the effective cooling velocity can now be obtained for each of the six wire orientations. Simultaneously solving any three adjacent equations provides expressions for the instantaneous values of the three velocity components $(u, v$, and $w$ in the facility $x, r$, and $\theta$ coordinates, respectively) in terms of the equivalent cooling velocities. It is then possible to obtain the three time-mean velocity components and the six different components of the Reynolds stress tensor in the manner described by Janjua 40 and Jackson. ${ }^{4}$ ? 
Jackson"1 assessed the accuracy and directional sensitivity of the technique, concluding that the configuration of probe vs local flow direction is of little importance. He recommended evaluating quantities from the average of all six posslble wire combinations, and this smoothing has been used exclusively in recent studles and the present study.

\section{RESULTS AND DISCUSSION}

The time-mean velocity and turbulence quantities for the three jet-to-crossflow velocity ratios $A=2,4$, and 6 are presented in Figures 5 through 8. Each flgure is composed of twelve plots, (a) through (1), of the data for one traverse angle $\theta$ at all seven axlal locations $(x / D$ $=1.00,1.25,1.50,1.75,2.00,2.50$, and 3.00). Using Figure 7 as an example, it can be seen that the time-mean and turbulent flowfields for the case of jet-to-crossflow velocity ratio $R=4$ and traverse angle $\theta=270$ degrees are presented. Recall that Figure 2 provides the geometrical relationship between the jet and the traverse angle $\theta$. For $\theta=270$ degrees, as in F1gure 7 , the vlewer is seelng an rx-plane of the flowfield which passes through the test section centerline and is normal to the lateral jet nozzle centerline. The top (bottom) of each plot corresponds to the first (last) measuring station, as shown in Figure 3. Tabulated values of the numbers used in these plots are given In Refs. 29 and 30.

In each of the figures, subparts $a, b$, and $c$ present normalized time-mean velocity components; subparts $d, e$, and $f$ give normalized rluctuating velocity components (normal stresses); subparts $\mathrm{g}$, $h$, and 1 exhibit normalized shear stresses; and subparts $j, k$, and $I$ provide normalized total velocity, axial turbulence intensity, and turbulent kinetlc energy, respectively.

The plots were produced on a Tektronix 4006 terminal connected to an IBM 3081D using PLOT 10 as the graphics control language. The data are merely scaled and plotted point-to-point for each axlal location. The $x / D$ scales also serve as the magnitude scale for each normalized data point. For example, in Figure $5 a$, the values of $\bar{u} / u$ at $x / D=1.00$ are scaled such that values of $\bar{u} / \mathrm{u}$. 1.00 are placed at $x / D=1.25$. In this figure, the values of $\bar{u} / u_{0}$ are very nearly 1.0 across most of the traverse.

\subsection{Jet-to-Crossflow Velocity Ratio $R=2.0$}

The velocity ratio $R=2$ is represented in Figure 5. It shows the best perspective to visualize the flowfield: the vertical rx-plane shown $(\theta=0$ degrees). It is the same as used for the flow visualization photographs, described elsewhere 5, 6, 29 and 30 . Part a of the flgure shows how the jet, which enters from the bottom of the plot at $x / D=1.00$, affects the axial velocity profiles. Figure 5b, the radial velocity plot, is interesting in that the six-position technique is capable of accurately measuring the jet velocity as It Impinges on the probe parallel to the probe axis. Note that the techniques can only produce magnitudes; there are no negative $\bar{v}$ velocities for instance. In Figure $5 a$ it can be seen that the $R=$ 2 case has virtually no effect on the mean flowfleld above the centerline.
The normal stresses, see parts $d, e$, and $f$ of Figure 5, are spread through the flowfield over a wider region than the mean velocity. These and the shear stresses in parts $8, h$, and 1 are very low in magnitude. Part 1 shows that the reglon of significant turbulent kinetic energy extends only a short distance. The total velocity magnitude, Figure $5 j$, is well-mixed and evenly distributed across the test section by $x / D=3.0$. These observations for the $R=2$ situation are not appropriate at higher values of $R$.

\subsection{Jet-to-Crossflow Velocity Ratio $R=4.0$}

The jet-to-crossflow velocity ratio $\Omega=4$ provides a flowfield whlch is more intricate than was the case when $R=2$. The centerline of the lateral jet crosses the centerline of the test section, and smoke and bubble flow visualization photographs ${ }^{6}$ show that the turbulent eddies are large compared to those of $R=2$. Figures 6 and 7 show profiles for the $R=4$ situation in the vertical $(\theta=0$ degrees $)$ and horlzontal

$(\dot{\theta}=270$ degrees $)$ planes, respectively.

Focusing attention on Figure 6a, which shows profiles in the vertical plane through the centerlines of both jets, it can be seen that the jet has a marked effect on the mean flowfleld below the jet centerline, causing axial flow deceleration. The axial velocity at $x / D=1.00$ shows that the upstream flow has slowed to go around the lateral jet on either side.

The fet centerline location can be deduced from radial locations of maximum total velocity, as shown in Figure $6 \mathrm{j}$. Because the jet centerine crosses through the facility centerline, Figure 7 $(\theta=270$ degrees) provides insight into the structure of the jet. First, parts $a, b$, and $c$ show good symmetry. The jet centerline was previously measured to cross the crossflow centerline at $x / D=1.35$ in Ref. 5. This is seen to affect the tangential and radial velocities first at $x / D=1.25$. The axial velocity is not changed until $x / D=1.50$ where it exhiblts a flat acceleration and then, at $x / D=1.75$, a deceleration in the mean velocity. This deceleration corresponds to an Increase in the surrounding axlal velocity outside of the jet. By $x / D=3.00$, the axlal velocity profile is relatively flat again. In reviewing the tangentlal velocity profiles in part $c$ of the figure, the most surprising feature is the symmetry and uniformity of the curves. At $x / D=1.75,2.00,2.50$, and 3.00 the "gull-wing" shape of the profiles is very likely caused by the data reduction results. That is, the profiles should actually look like "normal probablity distribution curves" -- if negative values could be obtalned. Physically this means that the tangential velocity along the central plane of the jet first rotates one direction, say clockw1se, outside of the jet, and then the other direction, say counter-clockwise, inside the jet. The normal stresses, Figures 7 parts $d$ and $e$, exhibit the expected peaks at the crossover. Note the dual peak in the u-direction normal stress, Figure $7 d$, at $x / D=1.75$.

There are some problems with the measurement technique and assumptions in signal interpretations in some regions of the flow. The normalized axial velocity in Figure 6a, for example, shows a large value below the jet centerline, $x / D=1.25$. The 

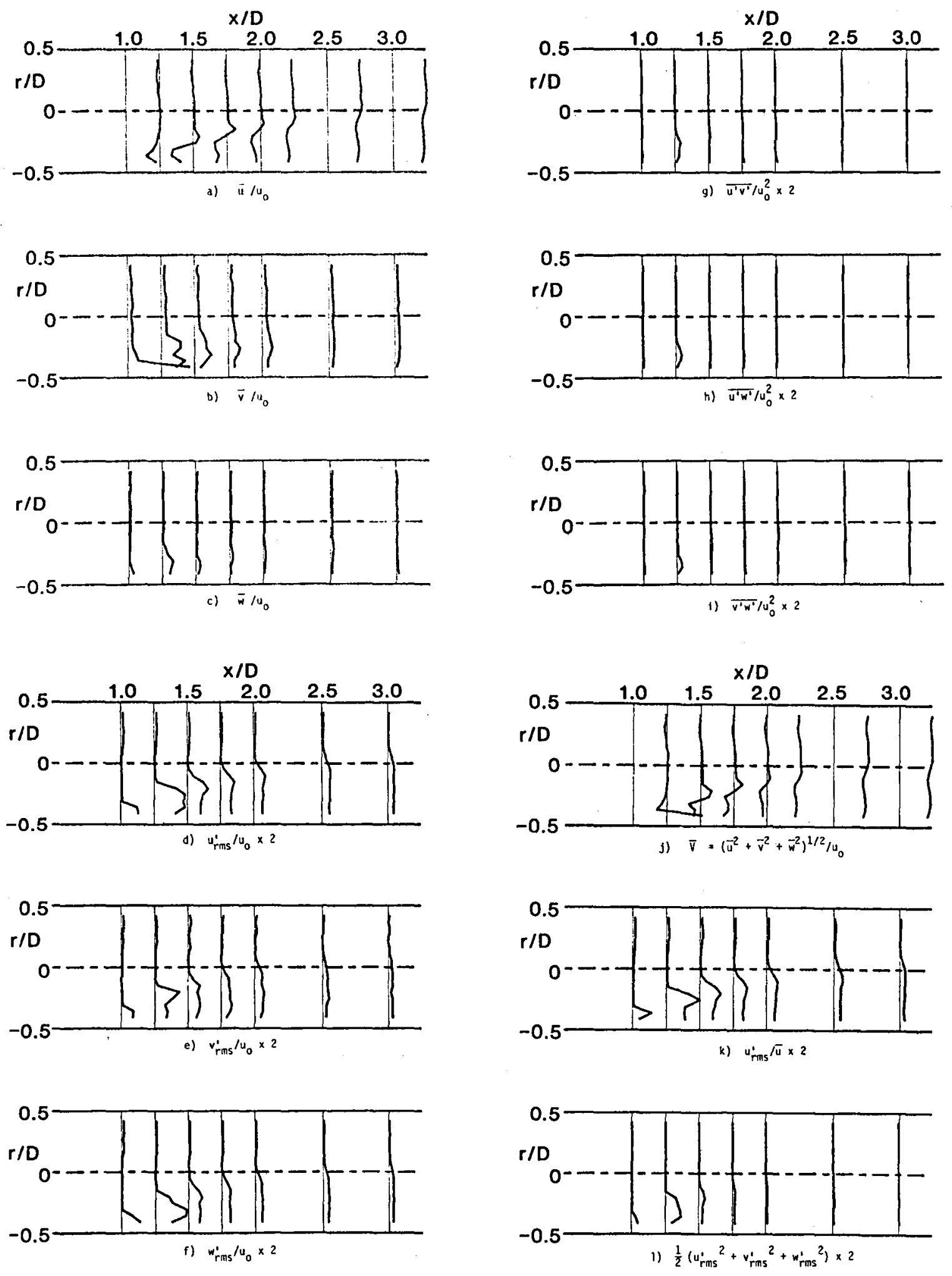

Fig. 5. Time-Mean and Turbulent Flowfield, $R=2.0$, Traverse Angle $\theta=0$ Degrees 

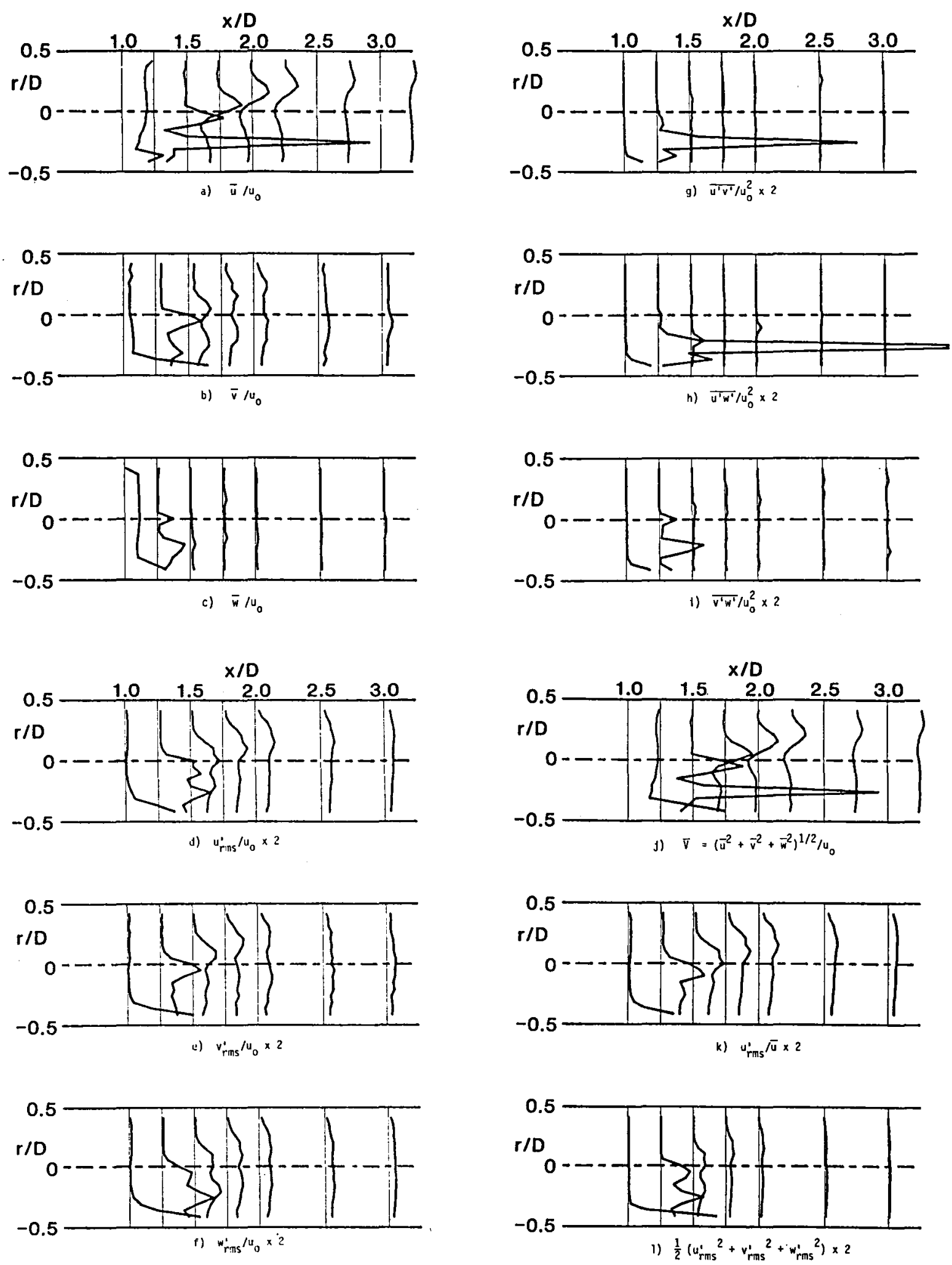

F1g. 6. Time-Mean and Turbulent Flowfield, $R=4.0$, Traverse Angle $\theta=0$ Degrees 

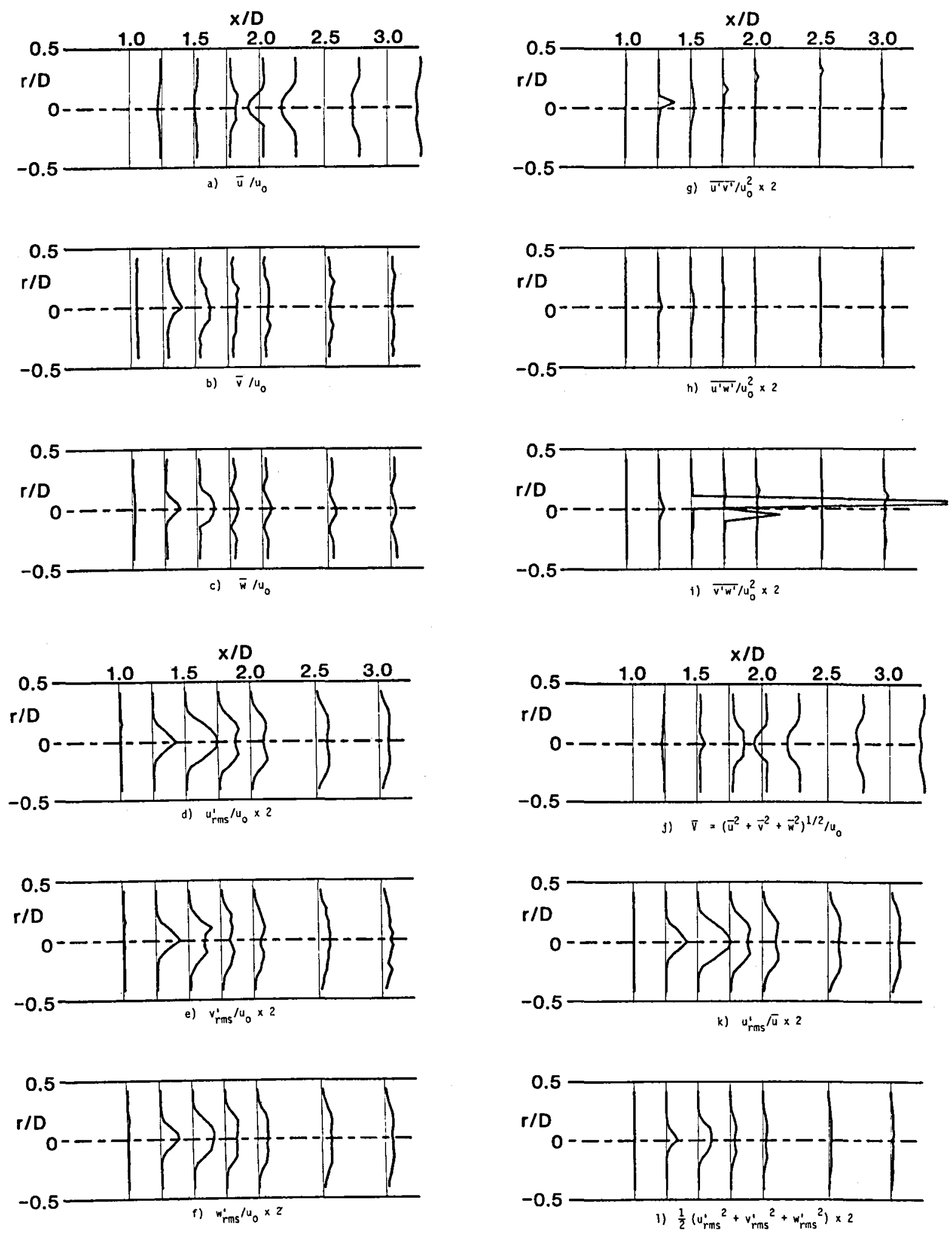

Fig. 7. Time-Mean and Turbulent Flowfleld, $R=4.0$, Traverse Angle $\theta=270$ Degrees 

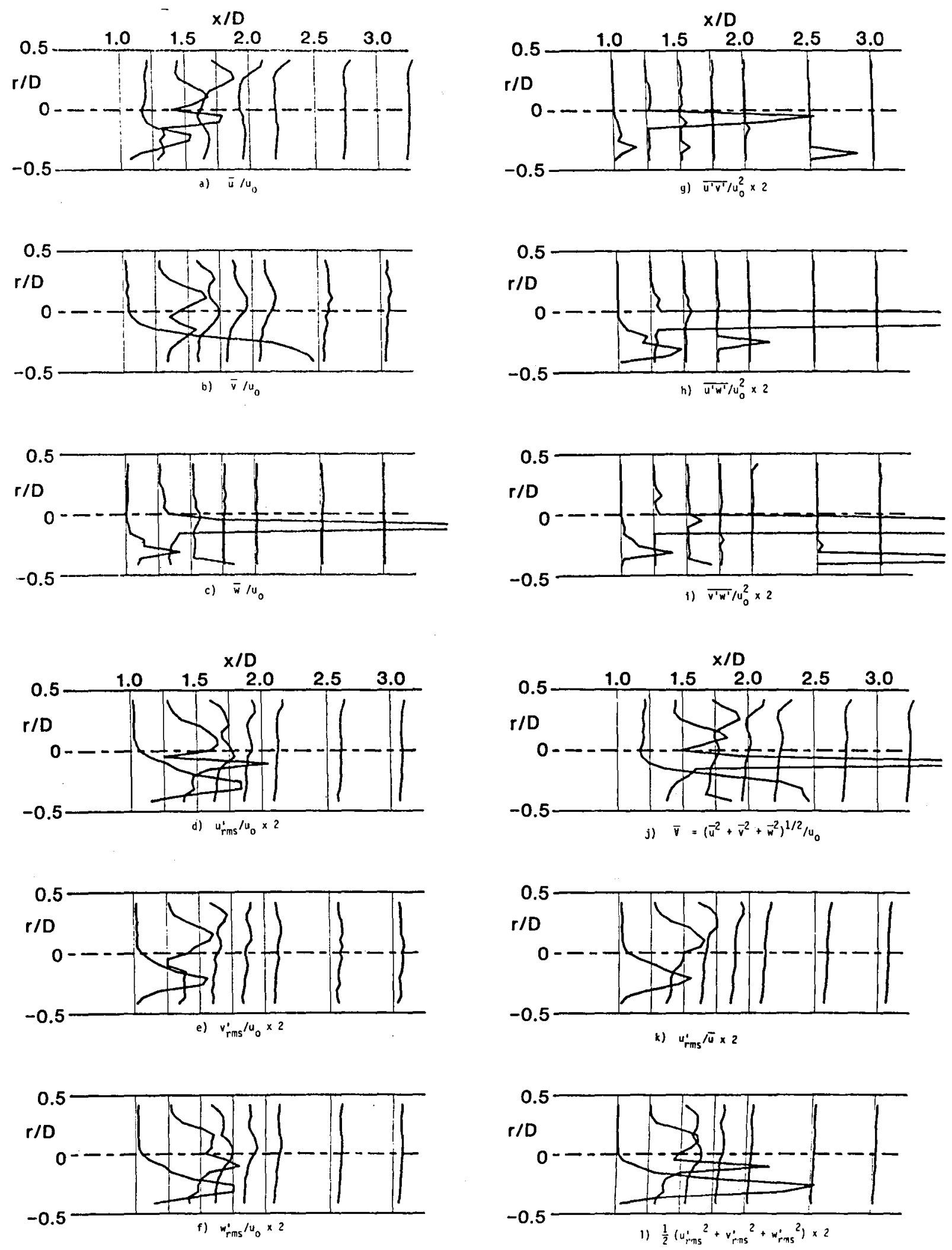

Fig. 8. Time-Mean and Turbulent Flowfield, $F=6.0$, Traverse Angle $\theta=0$ Degrees 
shear stress $\overline{u^{\prime} w} / u$ shows a very large and erroneous value at the same location, and is related to the erroneous value of the mean axtal velocity. These aberrant values have been falthfully presented along with the rest of the data.

\subsection{Jet-to-Crossflow Velocity Ratio $R=6.0$}

The jet-to-crossflow velocity ratio $R=6$ case dramatically exhibits some of the behavior expected of these deflected jets. Examining data in the horizontal plane, it is known ${ }^{29,30}$ that the jet centerline crosses the facility centerline at approximately $x / D=1.15$ to 1.25 . The axial velocity has a marked depression inside the jet and a large acceleration around the jet sides, as if the crossflow were passing a solid cylindrical obstruction.

As before, the plot that displays the data next in a most interesting format is the traverse in the vertical plane, with $\theta=0$ degrees, see Figure 8. As with the previous two cases, the axial velocity profile can be used to locate the jet centerline. However, the total velocity profile, given in part $j$ of the figure, is actually more accurate in locating the maximum velocity centerline. Providing testimony to the accuracy of the technique is Figure 8 , where the radial velocity $\bar{v} / u$ is seen to asymptotically approach the normalized lateral jet inlet velocity as the probe is lowered toward the jet exit throat. Unfortunately, the shear stress plots, given in parts $h$ and $i$ of the figure, show that the technique is very sensitive to erroneous readings and probably also to dwell time. The sensitivity of this technique to input varlables was discussed in section 4 and was the subject of analysis by Jackson. 41

\subsection{Jet Trajectories}

By using the maximum velocity magnitude to define the fet centerline, Figure 9 displays the comparative centerline locations for these experiments as compared to the infinite crossflow situation."2 As expected, the jet penetration for confined cylindrical situations is reduced from that of infinite crossflow situations, although low injection values correspond to cases for which the confining boundarles have little effect.

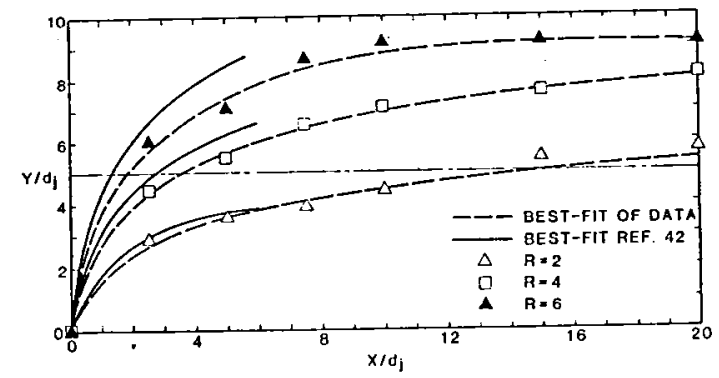

Fig. 9. Jet Centerline Locations for Different Jetto-Crossflow Velocity Ratios $R=2.0,4.0$, 6.0

\section{CLOSURE}

Experiments have been conducted to characterize the time-mean and turbulent flowfleld of a deflected turbulent jet in a confining cylindrical crossflow. Jet-to-crossflow velocity ratios of 2,4 , and 6 were investigated, under nonswirling crossflow conditions, using a sixposition single hot-wire technique. A computerlzed data acquisition and probe drive system was constructed to manipulate the hot-wire and facilitate the reduction of the time-varying slgnal to the statistical mean and root-mean-square voltage. A separate Fortran computer code was then used to reduce these voltage readings from several hot-wire rotation angles to the time-mean velocity and turbulent Reynolds stresses at a particular location. These velocities and stresses were then plotted for various rx-planes colnclding with the facility centerline.

Three entire flowfields were characterized via this technique for the jet-to-crossflow velocity ratios of interest. Measurements confirm, as expected, that the deflected jet is symmetrical (averaged over time) with respect to the vertical plane passing through the crossflow axis. The presence of the jet in the confines of the test section was seen to cause acceleration of the crossflow over and around the jet; and to slow the crossflow behind the jet, as if it were an obstruction. The jet penetration into the nonswirling crossflow was found to be reduced from that of comparable infinite crossflow cases, especially for the larger values ( 4 and 6 ) of the jet-to-crossflow velocity ratio. Normal and shear stress values were found to be largest in areas of strong jet and crossflow mixing, with evidence of reversal of tangentlal velocity components along jet mixing boundaries in the horizontal rx-plane of the racility.

\section{ACKNOHLEDGMENTS}

The authors express gratitude to NASA Lewis Research Center for assistance via Grant No. NAG 3549, technical monitor Dr. J. D. Holdeman. Help with the experiments came from several students, notably Carl B. MoMurry and Lawrence H. Ong, to whom special thanks are offered.

\section{REFERENCES}

1. Schetz, J. A., "Injection and Mixing in Turbulent Flow." Prog. In Astro. and Aero., AIAA, Vol. 68, 1980.

2. Lefebvre, A. H. "Gas Turblne Combustion." MoGraw-H1ll, New York, 1983.

3. Gupta, A. K., Lilley, D. G., and Syred, N. "Swirl Flows." Abacus Press, Tunbridge Wells, England, 1984.

4. Gupta, A. K. and Lilley, D. G., "Flowfleld Modeling and Diagnostics." Abacus Press. Tunbridge Wells, England, 1985.

5. Ferrell, G. B., Abujelala, M. T., Busnalna, A. A., Lilley, D. G., "Lateral Jet Injection Into Typlcal Combustor Flowfields." AIAA Paper 84-0374, Reno, Nevada, Jan. 9-12, 1984. 
6. Ferrell, G. B., Aoki, K. and Lilley, D, G. "Flow Visualization of Lateral Jet Injection Into Swirling Crossflow." AIAA Paper 85-0059, Reno, Nevada, Jan. 14-17, 1985.

7. Callaghan, E. E, and Ruggeri, R. S., "Investigation of the Penetration of an Air Jet Directed Perpendicularly to an A1r Stream." NACA TN 1615, 1948.

8. Ruggeri, R. S., Callaghan, E. E., and Bowden, D. T., "Penetration of Air Jets Issuing from Circular, Square and Elliptical Orffices Directed Normally to an Air Stream." NACA TN 2019, Feb. 1950.

9. Ruggeri, R. S., "General Correlations of Temperature Proflles Downstream of a Heated Air Jet Directed at Varlous Angles to an Air Stream." NACA TN 2855, 1952.

10. Jordinson, R., "Flow in a Jet Directed Normal to the Wind." Aero. Res. Council $R$ and $M$ No. $3074,1958$.

11. Keffer, J. F., and Balnes, W. D., "The Round Turbulent Jet in a Cross Wind." ASME Journal of Fluid Mechanics, Vol. 15, 1963, p. 481 .

12. Pratt, B. D., and Baines, W. D., "Proflles of the Round Turbulent Jet in a Cross Flow." Proceedings of ASCE, Journal of the Hydraulies Division, Nov. 1967, pp. 5663.

13. Pratt, B. D., and Kerfer, J. F., "Derlected Turbulent Jet Flows." Journal of Applied Mechanics, 1971, pp. 756-758.

14. Norgren, C. T., and Humenik, F. M., "Dilution Jet Mixing Study for Gas-Turbine Combustors." NASA TN-D-4695, Aug. 1968.

15. Ca pbell, J. F., and Schetz, J. A., "Analysis of Injection of a Heated Turbulent Jet Into a Cross-flow." NASA TR R-413, Dec. 1973.

16. Kamotani, Y., and Greber, I., "Experiments on Confined Turbulent Jets in Cross Flow." NASA CR-2392, March 1974.

17. Holdeman, J. D., and Walker, R. E., "Mixing of a Row of Jets with a Conflned Crossflow." AIAA Journal, Vol. 15, No. 2, Feb. 1977, pp. 243-249.

18. Chassaing, P., George, J., Claria, A., and Sananes, F., "Physical Characteristics of Subsonlc Jets in a Cross-Stream." Journal of Fluld Mechanics, Vol. 62, 1974, p. 4164.

19. Crabb, D., Durao, D. F. G., and Whitelaw, J. W., "A Round Jet Normal to a Cross-Flow", ASME J. of Fluids Engng., Trans., Vol. 103, 1981, pp. 142-153.
20. Rathgeber, D. E., and Becker, H. A., "Mixing Between a Round Jet and a Transverse Pipe Flow." Proceedings of 1st Symposium on Turbulent Flows, Penn State University, 1977.

21. Holdeman, J. D., Srinivasan, R., and Berenfeld, A., "Experiments in Dilution Jet Mixing." Paper AIAA-83-1201, Seattle, Wash., June 27-29, 1983, AIAA Journal, Vol. 22, Nov. 10, pp. 1436-1443.

22. Holdeman, J. D., Srinivasan, R., "On Modeling Dilution Jet Flowflelds." Paper AIAA-841379, Cincinnat1, On10, June 11-13, 1984.

23. Holdeman, J. D., "Dllution Zone Mixing." Presented at Combustion Fundamentals Research Conference, NASA Lewis Research Center, Cleveland, Ohio, oct. 21-22, 1982, pp. 106-118.

24. Srinivasan, R., Bernfeld, $A_{\text {., }}$ and Mongla, $H_{\text {, }}$ C. "Dilution Jet Mixing Program Phase I Report." NASA CR-168031, Nov. 1982.

25. Patankar, S. V., Basu, D. K., and Alpay, S. A., "Prediction of the Three-Dimensional Velocity Fleld of a Deflected Turbulent Jet." Journal of Flulds Engineering, Vol. 99, 1977, pp. 758-762.

26. Serag-Eldin, M. A., and Spalding, D. B., "Computation of Three-Dimensional Gas Turbine Combustion Chamber Flows." ASME 78-GT-142, London, England, 1978.

27. Swithenbank, J., Turan, A., and Felton, P. G., "3-Dimensional 2-Phase Mathematical Modeling of Gas Turbine Combustors." project SQUID (ONR) Workshop, Gas Turbine Combustor Design Problems, meeting held at Purdue University, $W$. Lafayette, Indlana, May 31-June 1, 1978.

28. Claus, R. W., "Analytical Calculation of a Single Jet in Cross-Flow and Comparison with Experiment." Paper AIAA-83-0238, Reno, Nevada, Jan. 10-13, 1983.

29. Ferrel1, G. B., "Deflected Jet Experiments in a Turbulent Combustor Flowfleld." Ph.D. Thesls, Oklahoma State University, Stillwater, Okla., Dec. 1984.

30. Ferrell, G. B. and Lilley, D. G., "Deflected Jet Experiments in a Turbulent Combutor Flowrleld." NASA CR-174863, Feb. 1985.

31. Lllley, D. G., "Investlgation of Flowelelds Found in Typlcal Combustor Geometries." NASA CR-3869, Feb. 1985.

32. Busnalna, A. A. "Transient Three-Dimensional Predictions of Turbulent Flows in Cylindrical and Cartesian Coordinate Systems." Ph.D. Thesis, Oklahoma State University, Stiliwater, Okla., July 1983.

33. Busnaina, A. A. "Translent Predictions of Lateral Jet Injection into Typical Isothermal Combustor Flowflelds." AIAA Paper 85-0144, Reno, Nevada, Jan. 14-17, 1985. 
34. Morel, T., "Comprehensive Design of Axisymmetric Wind Tunnel Contractions", ASME Paper 75-FE-17, Minneapolis, MN, May 5-7, 1975.

35. Rohrer, J. B., "The Deslgn and Assembly of a Probe-Drive Mechanism", MAE 4010 Project Report, School of Mechanical and Aerospace Engineering, OKlahoma State University, Stillwater, OK, May 1984.

36. Mitchell, S. L., "Design and Construction of an Analog to Digital Conversion System for a Hot-wire Anemometer in Turbulent Flow", M.S. Report, School of Mechanlcal and Aerospace Englneering, Oklahoma State University, Stillwater, OK, May 1984.

37. King, C. F., "Some Studies of Vortex Devices Vortex Amplifier Performance Behavior," Ph.D., Thesis, University College of Wales, Cardiff, Wales, 1978.

38. Drorak, K. and Syred,N., "The Statistical Analysis of Hot Wire Anemometer Signals in Complex Flowflelds," Paper presented at DISA Conference, University of Lelcester, England, 1972.

39. Jorgensen, F. E., "Directional Sensitivity of Wire and Fiber Film Probes," DISA Information No. 11, Franklin Lakes, N. J., May 1971, pp. 31-37.

40. Janjua, S. I., "Turbulence Measurements in a Complex Flowfield Using a Six-Orientation Hot-Wire Probe Technique", M.S. Thesis, Oklahoma State University, Stillwater, Okla., Dec. 1981.

41. Jackson, T. W., "Turbulence Characteristics of Swirling Flowflelds", Ph.D. Thesls, Oklahoma State University, Stillwater, OK, Dec. 1983.

42. Patankar, S. V. "Numerlcal Heat Transfer and Fluid Flow." Hemisphere-McGraw-H11l, New York, 1980. 
APPENDIX D

HOT-WIRE MEASUREMENTS OF A SINGLE LATERAL

JET INJECTED INTO SWIRLING CROSSFLOW

AIAA-86-0055 


\author{
L. H. Ong, * C. B. McMurry,* and D. G. Lilley** \\ Oklahoma State University, \\ Stillwater, Oklahoma
}

\section{ABSTRACT}

Experiments have been conducted to document the time-mean and turbulent flowfield of a deflected turbulent jet in a confined swirling crossflow. The jet-to-crossflow velocity ratio of 4 was investigated with swirler vane angles of 45 and 70 degrees. A six-orientation single hot-wire technique was used to measure the velocities and turbulence properties of the flow. The results are presented in the form of $r-x$ plots to ald visualization of the fully three-dimensional flowfield. The swirl in the crossflow intensifled the local velocity at the location of the injected jet, which effectively reduced the jet-to-crossflow velocity ratio. This caused the trajectory of the injected jet to follow the path of the local flow direction of the crossfiow, and reduce its penetration into the crossflow. The time-mean velocity measurements using the hot-wire corresponded closely to pitot-probe data obtained in identical flow conditions. The lateral jet was found to deflect the axis of the precessing vortex core.

\section{NOMENCLATURE}

$A$
$D$
$d$
$R$
$\frac{R e}{V}=(u, v, w)$
$x, r, \theta$
$\delta$
$\phi$
$\theta$

cross-sectional area test section diameter nozzle diameter jet-to-crossflow velocity ratio Reynolds number time-mean velocity in facllity coordinates $\left(x-, r^{-}\right.$ $\theta-d$ irections)

axial, radial, azimuthal coordinates $\operatorname{Arctan}(u / w)$ swirl vane angle with respect to facility axis

traverse azimuthal angle

Subscripts

c

j

rms

relating to crossflow relating to lateral jet value at inlet to flowfield root-mean-squared

\section{Superscripts}

()

( )

time-mean average

fluctuating component

\section{INTRODUCTION}

\subsection{Lateral Jet Injection}

Lateral injected jets are widely used in combustors of gas turbine engines to provide sufficient air for complete combustion as well as to evenly $\mathrm{mix}$ and $\mathrm{cool}$ the hot combustion products before their introduction to the turbine, As expected, the placement, size and injection velocities of lateral jets greatly affect the performance of the combustor in particular, and of the whole engine in general.

Clearly, the flowflelds in such situations are turbulent, reacting, and fully three-dimensional. Design and development of these combustors usually require the building of expensive prototypes followed by painstaking experiments, requiring lengthy and costly research programs. Time and expense could be greatly reduced with the avallability of reliable computer codes for direct prediction of these compllcated flowfields. The development of these, a subject of intensive present-day research, requires the existence of an accurate data base with which to compare predictions and further develop mixing models, finite difference schemes, and iterative procedures for improved accuracy and efficiency. Several textbooks extensively review problems and progress in this area. ${ }^{-5}$

The present study adds to the detailed data base being obtained in the on-going experimental combustor flow research program at Oklahoma State University. A recent $\mathrm{Ph}$. D. thesis ${ }^{6}$ discusses hotwire measurements of a single lateral jet being injected into nonswirling crossflow, and presents flow visualization results concerning injection into swirling crossflow, with crossflow swirl vane angle $\phi=0$ (swirler removed), 45 and 70 degrees. Three research papers have also evolved ${ }^{-9}$ from the recent study. All flowflelds belng investigated under this program at Oklahoma State University have no expansion of the crossflow (the test section to swirler diameter ratio $\mathrm{D} / \mathrm{d}=1$ ), after its passage through an optional swirler, with swirl vane angle $\phi=0$ (swirler removed), 45 and 70 degrees. The lateral jet is located one testsection diameter downstream of the test section inlet $(x / D=1)$ and it emanates from a round nozzle whose area is $1 / 100$ th of the cross-sectional area of the crossflow $\left(A_{j} / A_{c}=1 / 100\right)$.

\section{2 objectives}

The present study concentrates on a single lateral jet being injected normaliy into swirling crossflow. It complements previous studies which focused on corresponding situations with swirl but without lateral injection, and with lateral injection but without swirl. ${ }^{\circ}$ It also complements a concurrent study investigating two opposed

This paper is declared a work of the U.S. 
lateral jets being injected into swirling crossflow. ${ }^{10}$ The present study investigates two particular flowflelds having lateral jet-tocrossflow velocity ratio $v_{j} / u_{0}=4$, with swirl vane angle $\phi=45$ and 70 degrees being used with the main crossflow. The six-orientation single-wire hot-wire technique was used to measure the three time-mean velocities, three normal and three shear turbulent stresses throughout the test section.

\subsection{Outline of the Paper}

Section 2 gives a brlef insight into the history and previous studies of the injection jet. Included is also an overview of the recent work at Oklahoma State University on combustor flowflelds that led to the present undertaking. The experimental set up used in the present study is described in section 3, with discussions on the wind tunnel, the vane swirler, the test section and the incorporation of the injection jet, as well as a brief discussion of the hot-wire instrumentation. The data acquisition technique using the single normal hot-wire is also described.

Section 4 presents and analyses the extensive results that were obtained during this investigation. Finally, section 5 concludes the present study and makes some recommendations for further work.

\section{BACKGROUND}

\subsection{Previous Studies of Deflected Jets}

Ferrel19 provides an extensive description and classification of significant earlier studies on deflected jets. These studies include experimental (pitot probe, hot-wire and laser anemometer methods) and theoretical (empirical relationships and direct solution of the approprlate governing equations) contributions. Most of them were concerned with a single lateral jet injected into 'infinite' uniform crossflow.

Chassaing et $\mathrm{l}^{11}$ compared several zones of similarity in injected jets. Laser doppler Investigations were conducted by $\mathrm{Crabb}$ et $\mathrm{al}^{12}$ on the velocity and turbulence flelds, especlally the vortex pairs generated by the crossflow being obstructed by the injected round jet. of more significance to combustion chamber applications was the work by Rathgeber and Becker. ${ }^{13}$ They measured the trajectory and mixing of injected jets into cylindrical crossflows. However, the study lacks turbulence data. Recent NASA sponsored experimental work led to empirical evidence about the effects of multi-injected jets into rectangular crossflow. The most recent published article about these studies is by Holdeman et a1. ${ }^{14}$

Significant is that these previous studies did not address the problem of injection into swirling crossflows which are typical of that occurring in combustors. As a contribution to this a research program was initiated at Oklahoma State University which built upon earlier experience without lateral injection jets. ${ }^{15}$ Ferrell ${ }^{5}$ completed his Ph. D. thesis on the single lateral injected jet with velocity ratios $R=v_{j} / u_{0}=2,4$ and 6 entering into nonswirling crossflow. The six-orientation single hot-wire measurement technique was used. In addition to this, several flow visualization techniques (bubbles, smoke and sparks) were used with these same velocity ratios into swirling crossflow, with swirl vane angle $\phi=0$ (swirler removed), 45 and 70 degrees. Detalled measurements were not made for these swirling cases, leading to the objectives of the present research to emphasize the swirl effect, via the five-hole pitot probe and the hot-wire measurement methods, on the single lateral injection jet with $R=4$ only. Ferrell and colleagues wrote conference papers dealing with preliminary results and computer simulation of the fully 3-D flowfield, " flow visualization studies, ${ }^{B}$ and the detailed hot-wire measurements. 9 concurrent research effort on two opposed lateral jets, each with $R=4$ entering the 0,45 and 70 degree swirling crossflow, is being undertaken by McMurry. 10

\subsection{Recent Work at Oklahoma State University}

Lilley's summarizes recent extensive experimental and theoretical research which has been completed at Oklahoma State University on 2-D axisymmetric geometries under low speed, nonreacting, turbulent swirling flow conditions, in the absence of any lateral jets. The flow enters the test section and proceeds into a larger chamber (the expansion $D / d=2$ ) via a sudden or gradual expansion (side-wall angle $\alpha=90$ and 45 degrees). A weak or strong nozzle may be positioned downstream to form a contraction exit to the test section. Inlet swirl vanes are adjustable to a varlety of vane angles with values of $\phi=0$, $38,45,60$ and 70 degrees being emphasized. The objective was to determine the effect of these parameters on isothermal flowfleld patterns, timemean velocities and turbulence quantities, and to establish an improved simulation in the form of a computer prediction code equipped with a suitable turbulence model. Experimental developments included:

1. Flow visualization was accomplished via still and movie photography of neutrallybuoyant helium-filled soap bubbles, smoke produced by an injector and multisparks across the flowfield.

2. Time-mean velocities have been measured with a five-hole pitot probe for a complete range of swirl strengths.

3. Turbulence measurements have been completed on swirling (up to $\phi=70$ degrees) as well as nonswirling flows using a six-orientation single-wire hotwire technique enabling all Reynolds stress components to be deduced.

Theoretical developments included:

1. An advanced computer code has been developed and improved to predict corresponding confined swirling flows to those studied experimentally.

2. Tentative predictions have now been supplemented by predictions made from realistic inlet conditions for a complete range of swirl strengths with downstream nozzle effects.

\section{EXPERIMENTATION}

\subsection{Wind Tunnel And Vane Swirler}

Figure 1 shows a schematic of the test facility which is described at length elsewhere. It consists of a wind tunnel, a variable-angle vane swirler, and a plexiglass test section of diameter $15 \mathrm{~cm}$. The wind tunnel has an axlal-flow fan whose 


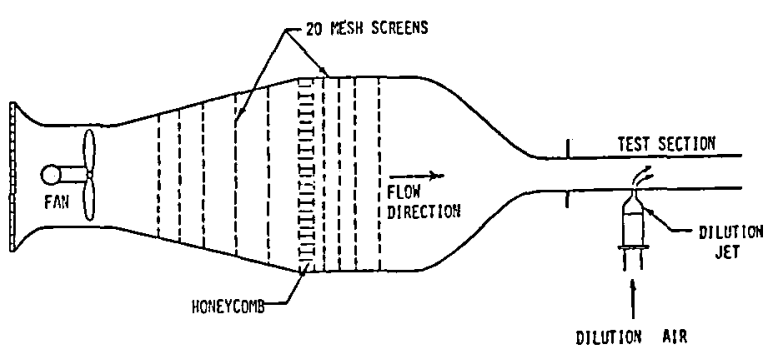

Fig. 1. Schematic of Experimental Facility
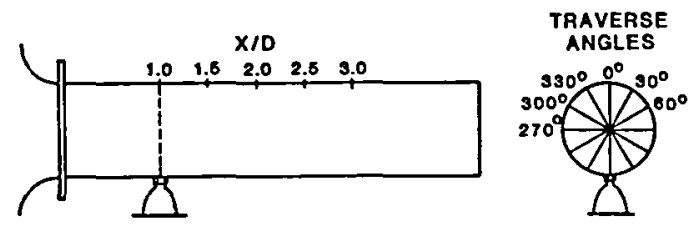

Fig. 2. Test Section Geometry

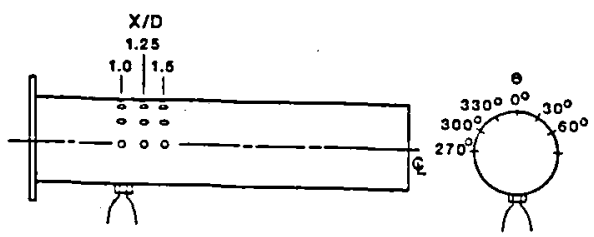

A. FIXED TUBE

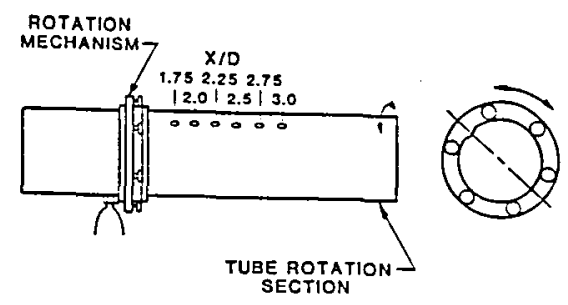

B. rotation tube

F1g. 3. Test Sections speed can be changed by altering a varidrive mechanism. Numerous fine screens and straws produce flow in the settling chamber of relatively low turbulence intensity. The contraction section leading to the test section has been designed by the method of Morel ${ }^{16}$ to produce a minimum adverse pressure gradient on the boundary layer and thus avold unsteady problems associated with local separation regions. The air-flow passes through a $15 \mathrm{~cm}$ diameter circular jet nozzle, exiting into a $15 \mathrm{~cm}$ diameter test section of length $90 \mathrm{~cm}$, which is constructed of plexiglass to facilitate flow visualization.

A variable-angle vane swirler may be positioned immediately before the test section. Its flow area has an outer diameter of $15 \mathrm{~cm}$. The swirler has ten vanes which are individually adjustable to any angle $\phi$ and $a$ hub with a streamlined upstream nose and a flat downstream face. The nose has a hyperbolic shape with a very smooth surface so as to offer minimal flow interference. The flat blades are wedge-shaped to give a constant pitch-to-chord ratio of 0.68 which gives good turning efficiency. ${ }^{3}$ Its performance is documented elsewhere. ${ }^{15}$ The test section normally begins at a location $x / D=0$, which is $3.2 \mathrm{~cm}$ downstream of the swirler exit.

Figure 1 also shows the configuration of the $15 \mathrm{~cm}$ diameter test section mounted on the wind tunnel with the swirler omitted. Air to the lateral jet is supplied from a compressed air line via piping and a carefully-designed nozzle. Upstream of each nozzle, a stagnation chamber, turbulence management screens, flow straightening straws, and flow metering equipment are used for flow conditioning. Experiments are being performed with the lateral jet located one test-section dlameter downstream of the inlet.

\subsection{Test Section and Dilution Jet}

clear acrylic tubing is used for the test section. of length $90 \mathrm{~cm}$, it is attached to the wind tunnel throat or immediately after the optional swirler. Standard commercial acrylic tube is used with $15.24 \mathrm{~cm}(6.0$ in.) outside diameter, $0.318 \mathrm{~cm}(0.125 \mathrm{in.})$ wall thickness. The inside diameter is $14.61 \mathrm{~cm}$ with a measured variation of $\pm 0.05 \mathrm{~cm}$. To adapt the test section to the wind Eunnel throat (inside diameter $15 \mathrm{~cm}$ ), an adaptor section was machined to provide a smooth transition from wind tunnel throat to test section. Two testsection tubes were constructed. Both test sections have the dilution jet inlet located at $x / D=1.00$ where $x$ is measured from the tube inlet. The first tube has a series of probe access holes located at $x / D=1.00,1.25$, and 1.50 and at all six azimuthal locations $270,300,330,0,30$ and 60 degrees as shown in Figure 2. The second tube allows probe access to locations downstream of $x / D=1.50$ (for example $x / D=1.75,2.00,2.50,3.00$ ) and at any azimuthal angle. This is accomplished via a tube rotation section, shown in Figure 3 , and constructed from machined aluminum rings, acrylic, and ball bearings.

The lateral jet is fed from laboratory compressed air at 6 to 7 atmospheres gauge pressure. For stability, the supply air lines are large and are routed through two line regulators with an intermediate tank (volume approximately $0.006 \mathrm{~m}^{3}$ ) to dampen line oscillations. The second 
regulator is used to meter the flow rate. After the second regulator, the air is routed through a Fisher and Porter mode1 10A1735A rotometer for monitoring of the volume flow before introduction to the dilution jet. The dilution jet assembly consists of a stagnation chamber, flow stralghtening section, and the jet nozzle. The stagnation chamber was constructed from $15 \mathrm{~cm}$ inside diameter aluminum pipe and filled with plastic household scrub pads to evenly distribute the internal flow. A hemispherically-shaped screen and convergent transition smooth the flow into the flow straightening section. Here the air flows through four brass screens for turbulence reduction. The nozzle is constructed of fiberglass with its precise contour being determined according to More ${ }^{16}$ in a manner similar to that used for the main wind tunnel facility. The nozzle dlameter is one-tenth of the test-section diameter, giving a crossflow to jet area ratio $A_{c} / A_{j}=100$. Once assembled, the dilution jet was attached to the air line coming from the rotometer, and the nozzle was pressed into a special acrylic adaptor which is permanently attached to the test section.

\subsection{Hot-Wire Instrumentation}

A normal hot-wire probe, DISA type 55P01, is used as the sensing transducer. It has two prongs set $3 \mathrm{~mm}$ apart with a $5 \mu \mathrm{m}$ dlameter tungsten wire between them. The exposed, effective length of the wire is approximately $1 \mathrm{~mm}$, since the ends have been gold plated to strengthen the wire and reduce end effects. The probe support is a standard DISA 55H21 straight mounting tube. The anemometer used is a DISA type 55Mø1, constant-temperature standard bridge.

Calibration of the hot-wire in three mutually perpendicular directions is done using the laboratory calibration jet - a small axisymmetric free jet. An extended King's law calibration expression is used. An automated computer controlled probe drive is used for data acquisition. Hot-wire voltages are processed through a Burr-Brown SDM853 12-bit A/D converter and the average and standard deviation of 5000 readings (taken over 5 seconds for each orientation of the wire at each measurement location) are stored on diskette using an Apple II computer. Ferrell ${ }^{6}$ provides greater detail about the instrumentation, calibration, probe drive and data acquisition systems.

\subsection{Data Acquilistion Technique}

When turbulence data are needed in addition to time-mean values, pitot probes are inadequate their response time is too slow. A more involved technique is then required, using hot-wire or laser doppler anemometry. A novel technique developed at Oklahoma State University for swirl flows without lateral injection is the six-orientation singlewire hot-wire method. ${ }^{15}$ It is very cost effective for flows of unknown dominant direction and has already been used on the nonswirling flow with lateral injection. ${ }^{-9}$ It is used in the present work without further refinement. Instrumentation used with this measurement technique has been described in Section 3.3. The six-orientation technique permits measurements of time-mean velocity components and turbulence quantities in complex three-dimensional flowflelds. Applied in this study to nonreacting flowflelds, measurements of time-mean and root-mean-square voltages at six different orientations (each separated by a 30 degree probe support rotations) contain enough information to obtain the three time-mean velocities, the three normal and the three shear Reynolds stresses. At each location in the flow, there are six different values of each of the above quantities that can be deduced using six sets of measurements of three adjacent orientations. Ensemble averages of the output quantities from the six combinations of data appear to produce estimates with the best agreement with independent measurements. The accuracy of the hot-wire method discussed in Section 4, where 1t is pointed out that severe inaccuracies may be present especially in the deduced shear stress values. Further information about the technique appears in the studies of Janjua, Jackson and Ferrell which are described in Refs. 6 and 15.

\section{RESULTS AND DISCUSSION}

Hot-wire time-mean and turbulence data, and time-mean velocity measurements using a five-hole pitot probe were obtalned for swirling flow without expansion, and with a lateral injection jet to crossflow velocity ratio of $R=v_{j} / u_{0}=4$. The lateral jet is situated at $x / D=f$, which is one test-section diameter downstream of the testsection inlet plane. Full results are given in the M. S. thesis of Ong. ${ }^{2}$,

The swirl cases investigated were that of swirler vane angle of 45 degrees and 70 degrees, represented moderate and strong swirl respectively. An earlier study presents corresponding results with no swirl in the crossflow. ${ }^{\circ}$ Two previous studies give measurements with swirl in the crossflow, but without any lateral jet, using not'wire ${ }^{18}$ and pitot probe ${ }^{19}$ techniques. Lateral transverses, at traverse angles of $270,300,330$, 0,30 and 60 degrees, with the not-wire were conducted at $x / D=1.00,1.25,1.50,1.75,2.00$, 2.50 , and 3.00 . The test section geometry is shown in Figure 3.

Hot-wire results of the experiment with moderately swirling crossflow are shown in and Figures 4 through 9. Similarly, Figures 10 through 15 show the data obtained for the strongly swirling crossflow. The figures just mentioned are subdivided into 3 parts to present the normalized time mean velocity components. In the $M$. $S$. thesis of Ong, ${ }^{37}$ the normal and shear stresses are also presented. The accuracy of results for turbulence quantities - normal stresses and, especlally, shear stresses - is in doubt. Jackson ${ }^{17}$ discusses the quality of the hot-wire technique in a weakly swirling axisymmetric expanded flowfield with $\mathrm{D} / \mathrm{d}=$ 2. The conclusion is that in turbulent shear regions, the maximum errors are 18, 24, 29, and 98 percent for time-mean values, normal stresses, shear stresses and $\overline{u^{\prime} w^{\prime}}$, respectively. The present study emphasizes the stronger swirl without expansion into the test section and may have potentially further accuracy problems. One major limitation with the present data acquisition system is the limited sampling rate of $1000 \mathrm{~Hz}$. Thus, the higher frequency signals of the hot-wire would not be properly digitized. Normally, an appropriate filter would be used in the data acquisition system to reduce this allasing effect. The present study does not use such a filter. However, in this case, where the turbulence energies might be in excess of 

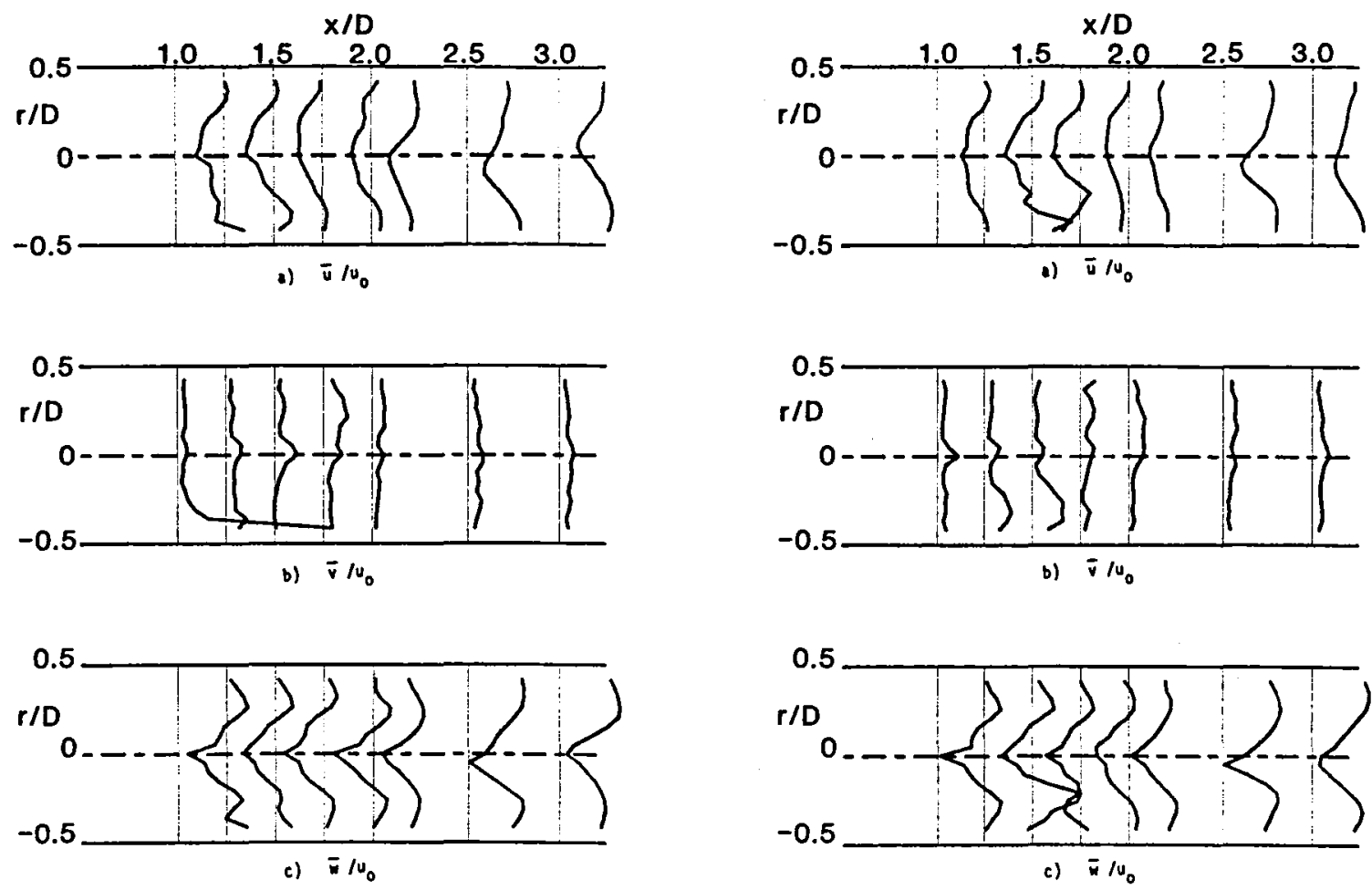

Fig. 4. Time-Mean Velocity with $\phi=45$ Degrees, Traverse Angle $\theta=0$ Degrees

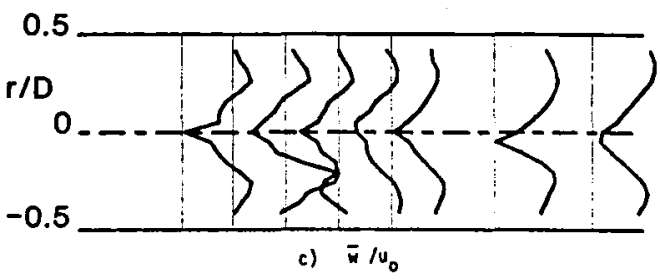

Fig. 6. Time-Mean Velocity with $\phi=45$ Degrees, Traverse Angle $\theta=300$ Degrees
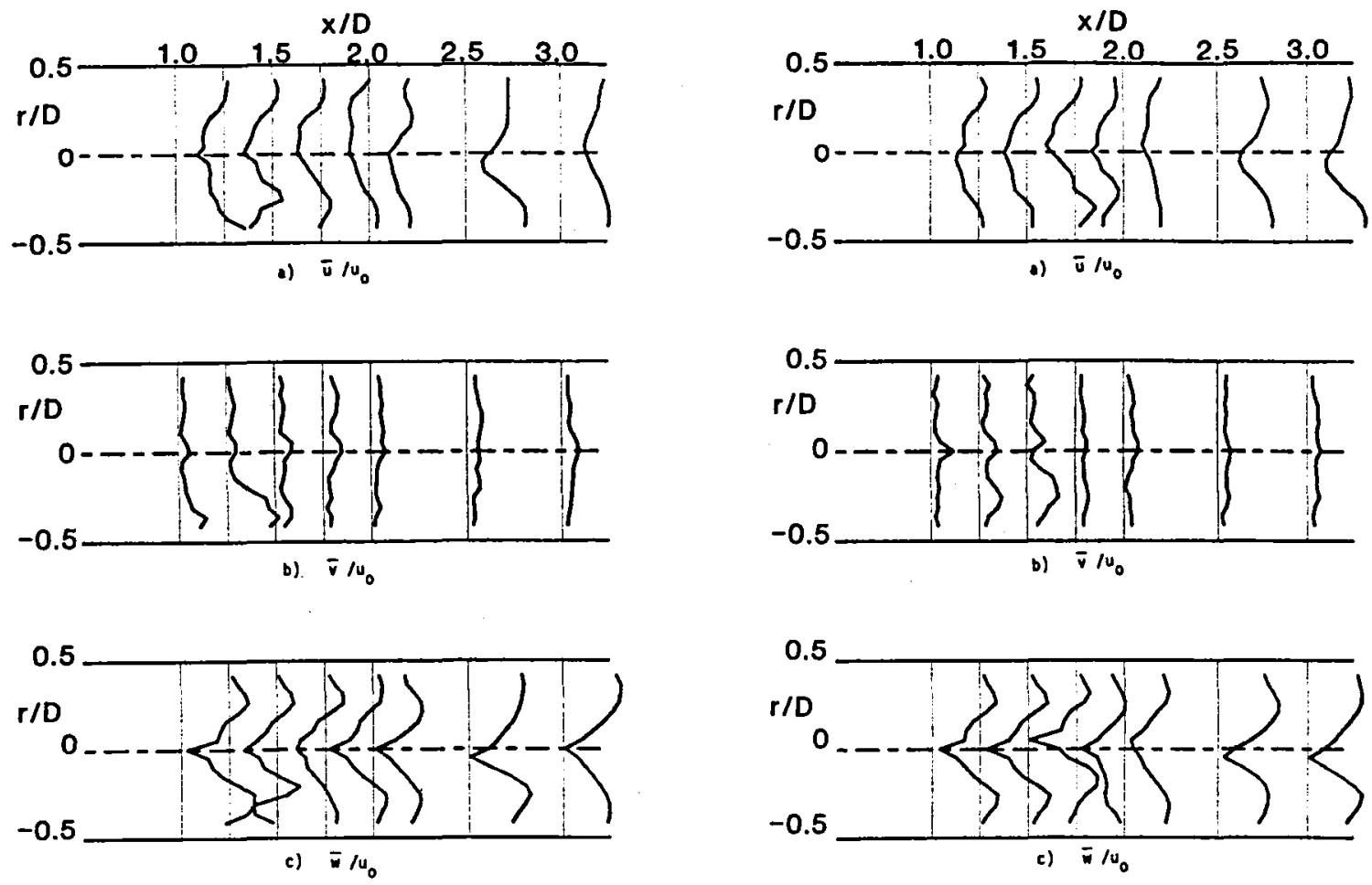

F1g. 5. Time-Mean Velocity with $\phi=45$ Degrees, Traverse Angle $\theta=330$ Degrees

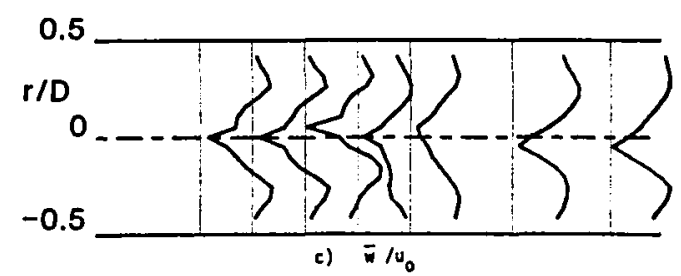

Fig. 7. Time-Mean Velocity with $\phi=45$ Degrees, Traverse Angle $0=270$ Degrees 

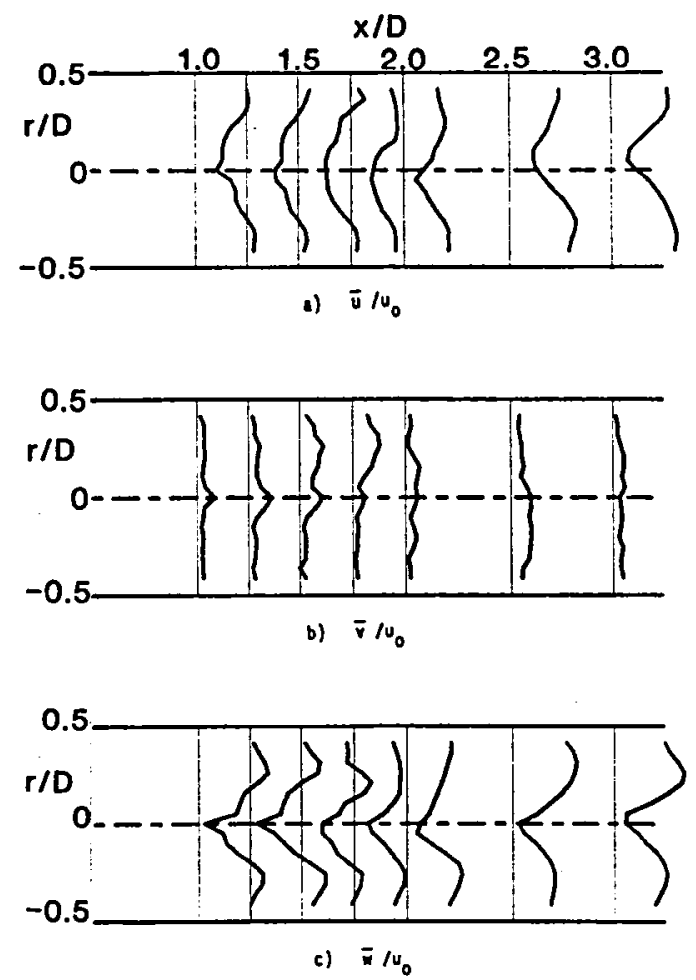

Fig. 8. Timë-Mean Velocity with $\phi=45$ Degrees, Traverse Angle $\theta=60$ Degrees
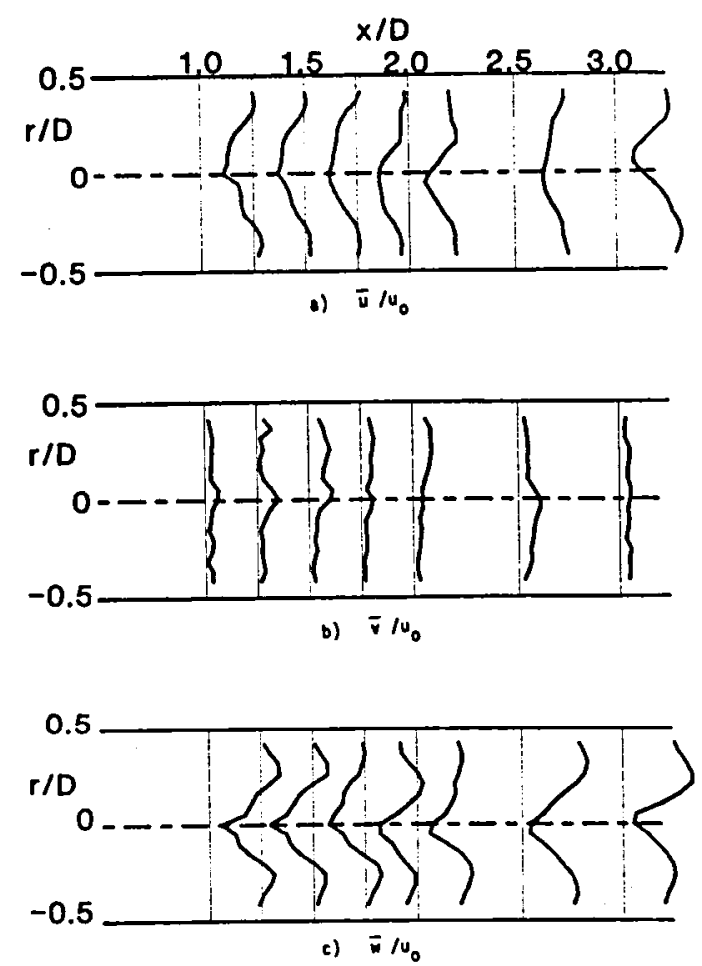

Fig. 9. Time-Mean Velocity with $\phi=45$ Degrees, Traverse Angle $\theta=30$ Degrees
$1000 \mathrm{~Hz}$, the lack of a filter might actually improve the data by inclusion of the allased high frequency signals. The amalgamated effect of these uncertainties leads to an unknown degree of error in the calculated values of turbulence quantities, especially the shear stresses. There are no alternative sources of data with which to compare the deduced values. For these reasons, the present paper presents only the time-mean velocity measurements, obtained with the hot-wire. Pitot probe time-mean velocity measurements were also obtained for the same swirling cases, 17 and results compare very favorably.

\section{1 Moderate SHirl, $\phi=45$ Degrees}

The entry of the injection jet can be clearly observed in parts $a, b$, and o of Figures 4 through 9, which show axial, radial, and swirl velocity plots, respectively. Even with this moderate swirl strength, the lateral jet is immediately swept along with the main flow in its swirling path after penetrating only up to $r / D=-0.4$ as shown in Figure 4 at $x / D=1.00$ (the inlet plane of the jet). This may be seen by inspection of the radial component of velocity $v$ at the location $x / D=1.00$ as shown in Figure $4 \mathrm{~b}$, which gives vertical traverses. The axial $u$ and swirl $w$ velocity components are increased in value close to the injection location along the path of the injected jet. For example, parts $a$ and $c$ of the vertical traverse of Figure 4 show this at the $x / D=1.00$ and 1.25 axlal locations.

The traverses presented in Figure 5 are aligned at 30 degrees to the vertical plane and give an indication of the path of the injected jet after injection, when the effects are no longer observable in the vertical plane. This rapid turning of the injected jet is expected as the swirl velocity component of the crossflow effectively increases the local velocity normal to the injected jet. This results in a reduced true jet-to-crossfiow velocity ratio and subsequently reduces the lateral jet penetration into the main flowfleld. For the 45 degree case, the $R$ value is reduced to approximately 2.8. Part a of Figure 5 shows enlarged axlal veloclty $u$ at $r / D=-0.25$ on the $x / D=1.25$ axial location; part $b$ of the same figure shows increased upward velocity $v$ near the same position; on the other hand, part $c$ shows reduced swirl velocity $w$ in the wake of the injected jet near this same location. Notlce that the swirl velocity nearer to the confining wall has not been affected since it is away from the path of the injected jet.

The angle between the local crossflow direction and the plane normal to the facllity centerline is provided by calculating $\delta=$ arctan $(u / w)$ locally. Near the lateral jet injection location this angle is approximately $\delta$ - 45 degrees for the moderate swirl $\phi=45$ degree case; it is approximately $\delta=27$ degrees for the strong swirl $\phi=70$ degrees case as discussed in Section 4.2. A spiralling path on the tube wall would require a normalized downstream distance of $L / D$ $=\pi(\tan \delta)$ to complete one revolution of the tube, if the angle $\theta$ remains constant. The trajectory of the lateral jet follows roughly this path, but the angle $\delta$ changes as the jet penetrates into the field. Hence, approximately, for the moderate swirl case of $\phi=45$ degrees, the required normalized downstream distance is $\mathrm{L} / \mathrm{D}=3.14$; for 

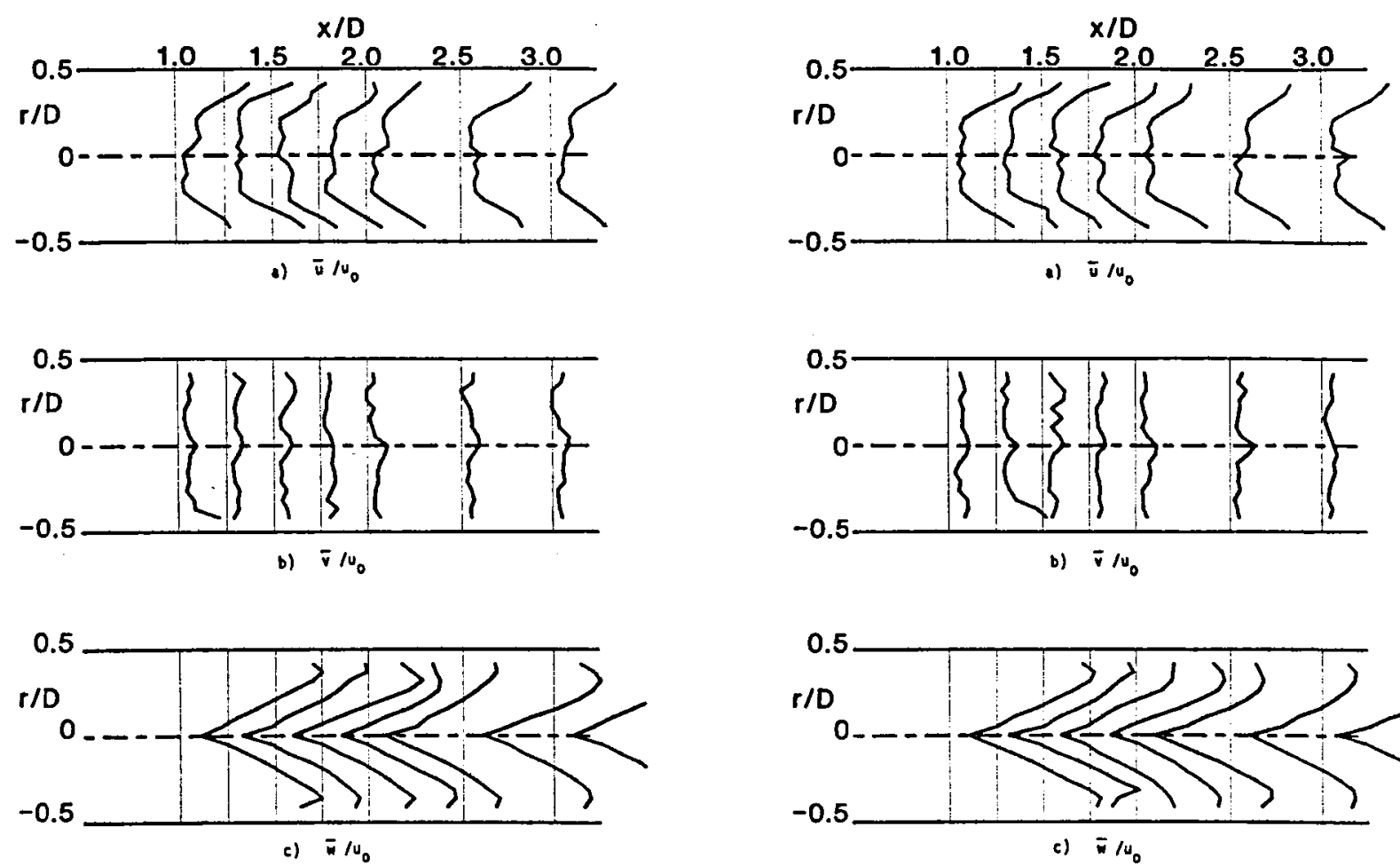

Fig. 10. Time-Mean Velocity with $\phi=70$ Degrees, Traverse Angle $\theta=0$ Degrees

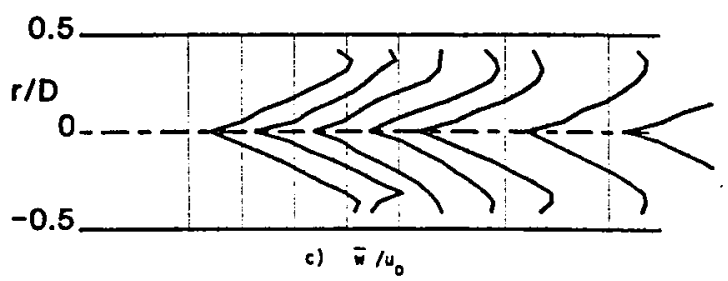

F1g. 12. Time-Mean velocity with $\phi=70$ Degrees, Traverse Angle $\theta=300$ Degrees
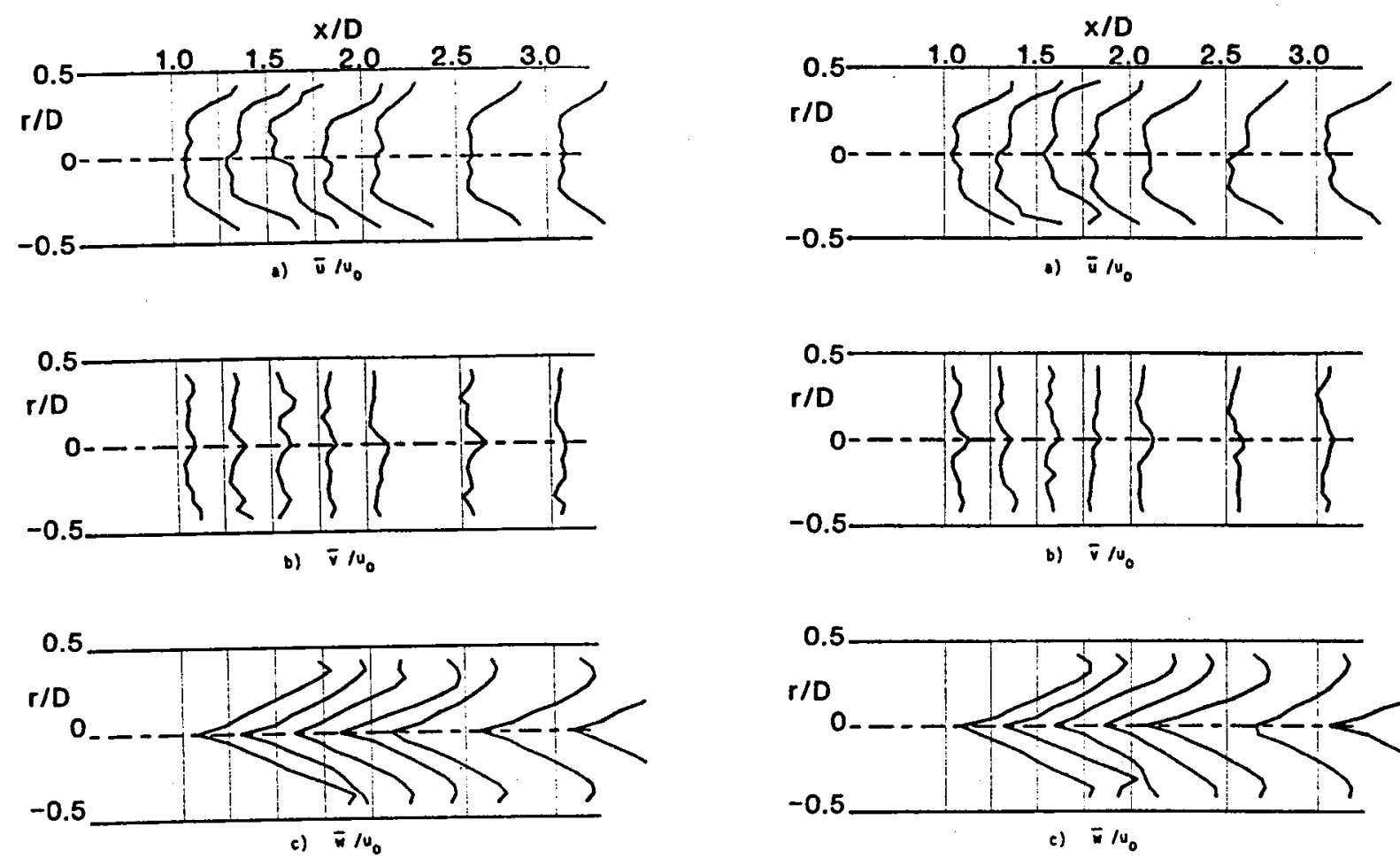

Fig. 11. Time-Mean Velocity with $\phi=70$ Degrees, Traverse Angle $\theta=330$ Degrees

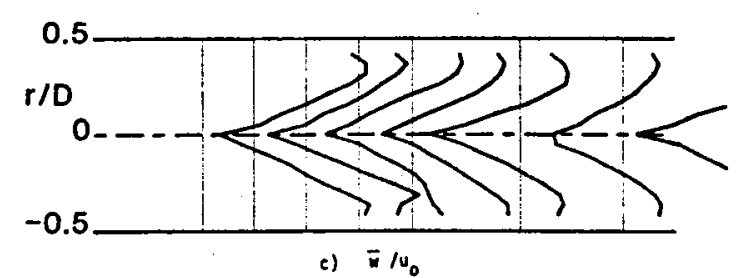

Fig. 13. Time-Mean Velocity with $\phi=70$ Degrees, Traverse Angle $\theta=270$ Degrees 

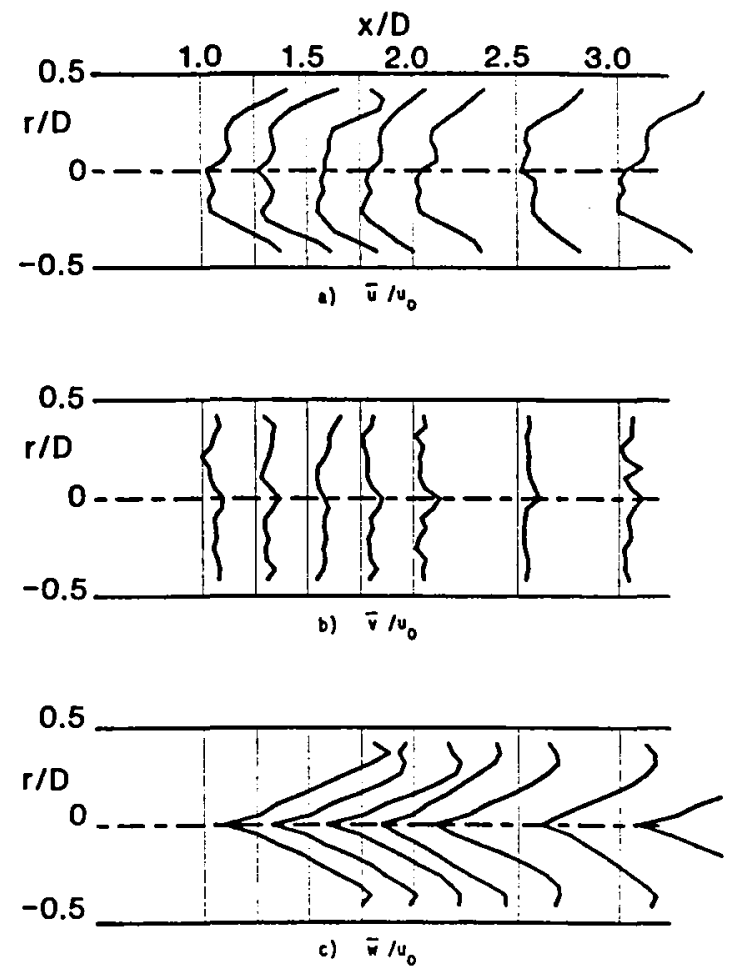

Fig. 14. Time-Mean Velocity with $\phi=70$ Degrees, Traverse Angle $\theta=60$ Degrees
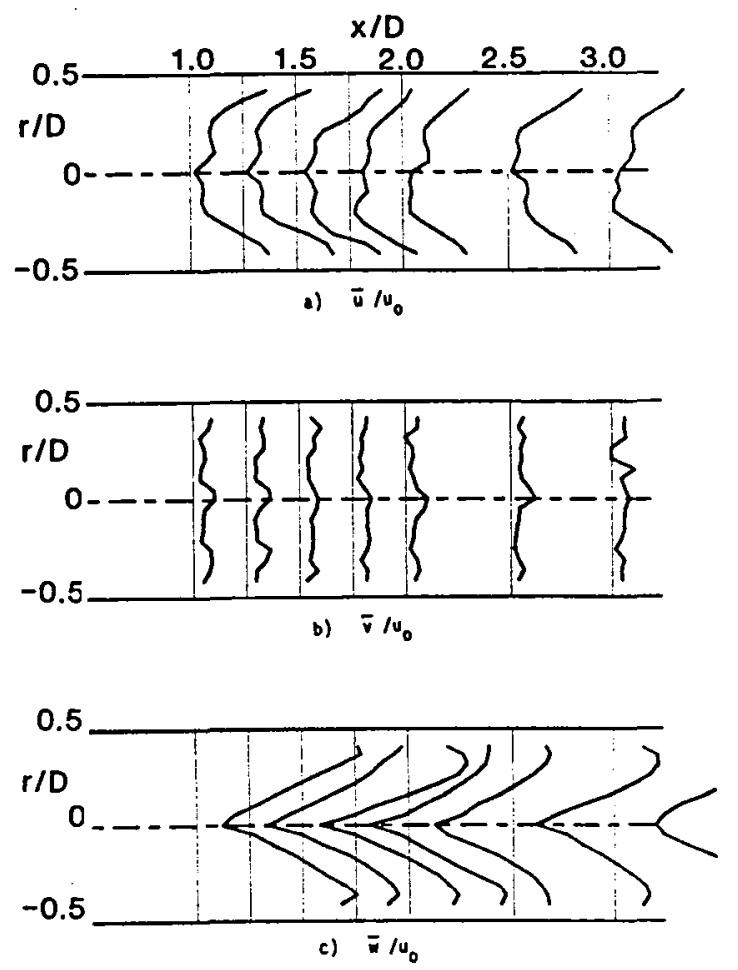

Fig. 15. Time-Mean Velocity with $\phi=70$ Degrees, Traverse Angle $\theta=30$ Degrees the $\phi=70$ degree case, it is $L / D=1.5$. Hence, evidence of the lateral jet is expected to be seen at $x / D=1.25$ in the $\theta=330$ degree traverse, at $x / D=1.5$ in the $\theta=300$ degree traverse, etc. for the moderate swirl case. For the strong swirl case, it is expected to be seen at $x / D=1.25$ in the $\theta=300$ degree traverse, at $x / D=1.5$ in the $\theta$ $=60$ degree traverse, etc.

The progress of the jet can be traced further downstream at $x / D=1.50$ by careful inspection of Figure 6 ( $a, b$, and $c)$, which are plots of the mean velocity data at a plane inclined at 60 degrees to the vertical. Simllarly, the increase in axial velocity component shown in Figure $7 \mathrm{a}$ at $\mathrm{x} / \mathrm{D}=2.00$ shows further progression of the jet. In this flgure, the spread of the injected jet can be deduced as one also notices larger $u$ and $v$ velocity components at $x / D=1.5$. It would seem that the traverse shown in Figure 6 , at $x / D=1.50$ obtained a velocity proflle near the center-line of the injected jet, while the traverse of Figure 7, at the same axial location would slice through the outer boundaries of the path of the injected jet. This evidence of the path and spread of the injection jet can be correlated to the bubble flow visualization photographs of the same flow conditions obtalned by Ferrell. ${ }^{6}$ After $x / D=2.00$, the spiral path of the jet, though not as pronounced, can still be traced by plcking out the locations where higher axial and total velocities are observed. For instance at $x / D=3.00$, the jet would appear to be near the top right quadrant of the test section, viewed towards the upstream direction. We can also deduce that the center-line is located near the bottom of the traverse of $\theta=$ 270 degrees as indicated by the higher mean axial velocity, shown in Figure 7 at $x / D=3.00$, when compared with the other traverse angle planes at the same axial location.

As expected, the introduction of the lateral fet into the crossflow destroyed the symmetry of the swirling crossflow. Jackson ${ }^{18}$ and Scharrer ${ }^{19}$ used the same hot-wire and pitot-probe measurement techniques, respectively, to measure the axisymmetric, swirling non-expanding flowfleld. Their data were used as a basis of comparison for the current experiments. The injected jet contributes less than 4 percent of the total mass flow, so that the flowfleld is not drastically changed from the axisymmetric case at locations away from the infected jet path, and after it has spread and mixed with the crossflow.

The disturbance of the precession vortex core noticed by Ferrell ${ }^{6}$ is also obvious in the mean axial and swirl velocity plots. The center-line of the vortex core is characterized by low axial and swirl velocities and hence its deflection can be observed, for example, in Figure 7 ( $a$ and $c$ ) at $x / D$ $=2.0,2.5$ and 3.0 , because the low velocity regions is not only away from the centerline of the test section but is also at different positions in each of the axial locations.

Higher turbulence intensitles, not shown here, were measured at locations where the intruding jet interacts with the main crossflow. ${ }^{17}$ However, the shear stress does not appear to be affected by the injection jet. This shear stress data shows the same trends as previous swirl flow data without lateral injection, obtained by Jackson. ${ }^{18}$ 


\subsection{Strong Swirl, $\phi=70$ Degrees}

As discussed in Section 4.1, the angle between the local crossflow direction and the plane normal to the facility centerline $\delta=\arctan (u / w)=27$ degrees for this strong swirl case near the lateral jet injection location. This requires approximately a downstream distance of $L / D=1.5$ for the path of the jet to ideally make one revolution, with evidence of the lateral jet expected to be seen at $x / D=1.25$ in the $\theta=300$ degree traverse, at $x / D=1.5$ in the $\theta=60$ degree traverse, etc.

The high swirl velocity of the crossflow in this case forces the injection jet to turn almost immediately into the local main flow direction. This is evidenced by the higher radial velocity component at $r / D=-0.4$ and $x / D=1.00$ of the vertical traverse of Figure 10 . More significantly, the path at the next axial location, $x / D=1.25$ appeared to be near $\theta=300$ degrees and $r / D=0.35$ as compared to that of $\theta=330$ degrees and $r / D=0.25$ of the moderate swirl case. This not only exhibits the rapid deflection of the injection jet but also indicated that the jet is sticking closer to the wall of test section due to higher centrifugal forces and also intensified jetto-local crossflow velocity ratio as discussed prevlously in the moderate swirl case. The local strong swirl velocity now reduces the $R$ value to approximately 1.2 which from previous work ${ }^{6}$ would lead to very little penetration of the lateral jet into the main stream. Because of rapid mixing in this high swirl strength, the path of the jet is not clearly identified after $x / D=1.50$. This fact is supported by the flow visualization photographs obtalned by Ferrell. ${ }^{\circ}$ The axisymmetric measurements for this swirl strength were also conducted by Jackson ${ }^{17}$ and Scharrer ${ }^{18}$ as described previously. The effect of the injection jet is even less pronounced than in the moderate swirl case because of higher swirl strength and increased mixing.

High turbulence intensities were again observed along the path of the injected jet. ${ }^{17}$ The shear stresses are again of very low magnitude and do not appear to have been affected by the injected jet. The normal and shear stress calculation using this hot-wire technique is error prone, as discussed earlier. This Idiosyncrasy was discussed by Ferrel1 ${ }^{6}$ and especially by Jackson. ${ }^{18}$

\section{CLOSURE}

\subsection{Conclusions}

Time-mean and turbulence measurements using a six-position single hot-wire technique have been completed for non-expanding swirling flowflelds that were disturbed by a single lateral injection jet of jet-to-crossflow velocity ratio $R=v_{f} / u_{0}=$ 4. The cases investigated consist of flowflelds with the upstream swirler vane angle set at 45 and 70 degrees, representing moderate and strong swirl respectively. Time-mean velocity measurements using a five-hole pitot probe technique were also conducted for the same conditions. The results were presented in the form of $\mathrm{r}-\mathrm{x}$ plots to help visualize the fully three-dimensional flowfleld.

Data from the moderate swirl case showed that the trajectory of the injected jet can be easily traced. The spreading of the injected jet can also be observed. The swirl in the crossflow intensified the local velocity at the location of the injected jet, which effectively reduced the jet-to-crossflow velocity ratio. This caused the trajectory of the injected jet to follow the path of the local flow direction of the crossflow, and reduced its penetration into the crossflow. The lateral jet was also found to deflect the axis of the precessing vortex core.

At the higher swirl strength, the turning of the jet into the local crossflow direction is more pronounced for reasons described above and higher centrifugal forces. The injection jet appears to disperse more rapidly in this case because of the high intensity of the mixing. The time-mean velocity measurements using the hot-wire corresponded to pitot-probe data ${ }^{17}$ obtalned under identical flow conditions.

\subsection{Recommendations for Further Work}

A useful addition to the data base generated by the present and concurrent study of two opposed injection jets by McMurry ${ }^{10}$ would be to investigate cases with different injection jet-to-crossflow velocity ratios of, for example, $R=v_{j} / u_{0}=2,6$, and 8 . The effects of additional jets could also be studled, as found, for example, in a practical case of a combustor with six inlets.

Use of a more advanced and complex hot-wire technique might improve the accuracy of measurements. For instance, a cross-wire or triple-wire probe could be used, but some of these data reduction techniques require a priori knowledge of local time-mean velocity, and more complicated probe handling systems. These methods might reduce the current dwell time measurement errors as multi-wire probes record correlation measurements almost simultaneously. However, there are problems with probe effects, interference and directional sensitivity of these multi-wire probes. Instrumentation of these probes is also far more complex. A useful Improvement of the present technique would be to conduct an energy spectrum analysis of hot-wire signals. This would enable the determination of the range of turbulence frequencies. Then a more accurate measurement would be obtained by increasing the sampling rate of the data acquisition system and appropriate filtering to reduce the effects of allasing.

\section{ACKNOWLEDGMENTS}

We are indebted to NASA Lewis Research Center, for support via Grant No. NAG 3-549, technical monitor Dr. James D. Holdeman.

\section{REFERENCES}

1. Schetz, J. A., "Injection and Mixing in Turbulent Flow", Prog. In Astro, and Aero., AIAA, Vol. $68,1980$.

2. Lefebvre, A. H. "Gas Turbine Combustion." MoGraw-H111, New York, 1983.

3. Gupta, A. K., Lilley, D. G., and Syred, N. "Swirl Flows." Abacus Press, Tunbridge Wells, England, 1985. 
4. Gupta, A. K. and Lilley, D. G., "Flowfield Modeling and Diagnostics." Abacus Press, Tunbridge Wells, England, 1985.

5. Cheremisinoff, N. P. and Gupta, R. (eds.) "Handbook of Fluids in Motion." Ann Arbor Science, Ann Arbor, Michigan, 1983.

6. Ferrell, G. B., "Deflected Jet Experiments in a Turbulent Combustor Elowfield." Ph.D. Thesis, Oklahoma State University, Stillwater, Okla., Dec. 1984. See also: Ferrell, G. B. and Lilley, D. G., "Deflected Jet Experiments in a Turbulent Combustor Flowfield." NASA CR-174863, Feb. 1985.

7. Ferrell, G. B., Abujelala, M. T., Busnaina, A A., and Lilley, D. G., "Lateral Jet Injection into Typical Combustor Flowfields." Paper AIAA-84-0374, Reno, Nevada, January $9-12,1984$.

8. Ferrell, G. B., Aoki, K. and Lilley, D. G., "Flow Visualization of Lateral Jet Injection into Swirling Crossflow." AIAA Paper 85-0059, Reno, Nevada, Jan. 14-17, 1985. See also: AIAA J. of Propulsion and Power, Vol. 1, No. 6, Nov.-Dec. 1985, pp. $485-487$.

9. Ferrell, G. B. and Lilley, D. G., "Turbulence Measurements of Lateral Jet Injection into Confined Tubular Crossflow." Paper AIAA85-1102, Monterey, Calif., July 8-10, 1985.

10. McMurry, C. B., "Experiments on Two Opposed Lateral Jets Injected into Swirling Crossflow." M.S. Thesis, Oklahoma State University, St1liwater, Okla., Dec. 1985.

11. Chassaing, P., George, J., Claria, A., and Sananes, F., "Physical Characteristics of Subsonic Jets in a Cross-Stream." Journal of Fluid Mechanics, Vol. 62, 1974, p. 4164.

12. 'Crabb, D., Durao, D. F. G., and Whitelaw, J. W., "A Round Jet Normal to a Cross-Flow." ASME Trans., Vol. 103, 1981, pp. 142-153.

13. Rathgeber, D. E., and Becker, H. A., "Mixing Between a Round Jet and a Transverse Plpe Flow." Proceedings of 1st Symposium on Turbulent Flows, Penn. State University, 1977.

14. Holdeman, J. D., Srinivasan, R., and Berenfeld, A., "Experiments in Dilution Jet Mixing." AIAA Journal, Vol. 22, No. 10, oct. 1984, pp. 1436-1443.

15. Lilley, D. G., "Investigation of Flowflelds Found in Typlcal Combustor Geometries." NASA CR-3869, Feb. 1985. See also: Lilley, D. G. "Swirling Flows in Typical Combustor Geometries." AIAA-85-0184, Reno, Nevada, Jan. 14-17, 1985.

16. Morel, T., "Comprehensive Design of Axisymmetric Wind Tunnel Contractions." ASME Paper 75-FE-17, Minneapolis, MN, May $5-7,1975$.
17. Ong, L. H., "Measurements of a Single Lateral Jet Injected Into Swirling Crossflow." M. S. Thesis, Oklahoma State Unlversity, Stillwater, Okla., Dec. 1985.

18. Jackson, T. W., "Turbulence Characterist1cs of Swirling Flowfields." Ph. D. Thesis, Oklahoma State University, Stillwater, Okla., Dec. 1983. See also: Jackson, T. W. and Lilley, D. G., "Turbulence Characteristics of Swirling Flowflelds." NASA CR-174918, May 1985.

19. Scharrer, G. L., "Swirl, Expansion Ratio and Blockage Effects on Confined Turbulent Elow." M. S. Thesis, Oklahoma State University, St1llwater, Okla. May 1984. 


\section{APPENDIX E}

TWO OPPOSED LATERAL JETS INJECTED INTO

SWIRLING CROSSFLOW

DRAFT PAPER JANUARY 1986 


\author{
C. B. McMurry,* L. H. Ong, * and D. G. Lilley** \\ Oklahoma State University \\ Stillwater, Oklahoma
}

\section{ABSTRACT}

Experiments have been conducted to obtain the time-mean and turbulent quantities of opposed lateral jets in a low speed, nonreacting flowfleld. A jet-to-crossflow velocity ratio of $R$ $=v_{g} / u_{0}=4$ was used throughout the experiments, with swirl vane angles of $\phi=0$ (swirler removed), 45 and 70 degrees used with the crossflow. Flow visualization techniques used were neutrallybuoyant hellum-filled soap bubbles and multi-spark photography in order to obtain the gross flowfield characteristics.. Measurements of time-mean and turbulent quantities were obtained utllizing a sixorientation single hot-wire technique. For the nonswirling case, the jets were found not to penetrate past the test-section centerline, in contrast to the single lateral jet with the same jet-to-crossflow velocity ratio. In the swirling cases, the crossflow remains in a narrow region near the wall of the test section. The opposed jets are swept from their vertical courses into spiral trajectorles close to the confining walls. Extensive results are presented in $r-x$ plane plots.

\section{NOMENCLATURE}

$\begin{array}{ll}A & \text { cross-sectional area } \\ D & \text { test section diameter } \\ \text { nozzle diameter } & \text { jet-to-crossflow velocity ratio } \\ R & \text { Reynolds number } \\ \text { Re } & \text { time-mean velocity in facility } \\ \bar{V}=(u, v, w) & \text { coordinates }\left(x^{-}, r-\theta \text {-directions) }\right. \\ x, r, \theta & \text { axial, radial, azimuthal coordinates } \\ \delta & \text { Arctan (u/w) } \\ \phi & \text { swirl vane angle with respect to } \\ \theta & \text { facility axis } \\ & \text { traverse azimuthal angle }\end{array}$

Subscripts

c relating to crossflow

$\mathrm{j}$ relating to lateral jet

o value at inlet to flowfield

rms root-mean-squared

\section{Superseripts}

(一)

( )

time-mean average

fluctuating component

\section{INTRODUCTION}

\subsection{Preamble}

In both can and annular combustion chambers lateral jets of cooler air are injected into the flowfleld through round holes in the combustor

* Graduate Student, School of Mechanical and Aerospace Engineering, Student Member AIAA

* Professor, School of Mechanical and Aerospace Engineering, Associate Fellow AIAA. walls. These jets enhance the performance of the combustor by altering the aerodynamics. In the swirl-induced central recirculation region of the primary zone, where most fuel burning takes place, additional air is provided by some of the lateral jets for stoichiometric conditions. In the secondary zone, other jets provide additional air to help complete combustion. Similar jets cool and evenly mix the products of combustion in the dilution zone before the flow enters the turbine. Similar processes occur in ramjet combustors. The complex nature of these flowflelds has resulted in the design and development of combustors being based largely on experimental trial and error research programs.

Recently, however, there has become increased emphasis on theoretical modeling of combustor flows, the success of which will lead to reduced time and cost of experimental research programs. Their development requires the existence of an accurate data base (for a variety of flow types) with which to compare predictions and further develop models for improved accuracy. Problems and progress in this area are extensively reviewed in recent textbooks. ${ }^{1-4}$

Research in progress at Oklahoma State University is concerned with combustor flowflelds in the absence of combustion. The experimental results being obtained add to the detalled data base. A recent $P h$. D. thesis ${ }^{5}$ discusses hot-wire measurements of a single lateral jet being injected into nonswirling crossflow, and presents flow visualization results concerning injection into swirling crossflow, with crossflow swirl vane angle $\phi=0$ (swirler removed), 45 and 70 degrees. Results with lateral jet to crossflow velocity ratio $\mathrm{R}=\mathrm{v}_{j} / \mathrm{u}_{0}=2,4$ and 6 were presented in all cases investigated. Three research papers have also evolved ${ }^{-8}$ from the recent study. All flowflelds being investigated under this program at Oklahoma State University have no expansion of the crossflow (the test section to swirler diameter ratio $\mathrm{D} / \mathrm{d}=1$ ), after its passage through an optional swirler (with swirl vane angle $\phi=0$ (swirler removed), 45 and 70 degrees). The lateral jet(s) is(are) located one test-section diameter downstream of the test-section inlet $(x / D=1)$. The lateral jets have round nozzles, each of which has an area of $1 / 100$ th of the cross-sectional area of the crossflow $\left(A_{j} / A_{c}=1 / 100\right)$.

\subsection{The Present Contribution}

Two opposed lateral jets injected normally into swirling crossflow is the focus of the present study. It complements a concurrent study investigating a single lateral jet being injected normally into swirling crossflow, ${ }^{9}$ and previous studies on similar configurations with swirl but without lateral injection, 10,11 and with lateral injection but without swirl.5 The present research investigates three particular flowfields having lateral jet to crossflow velocity ratio $R=v_{j} / u_{0}=$ 4 only, with swirl vane angle $\phi=0$ (swirler removed), 45 and 70 degrees being used with the 
main crossflow. Specific objectives included:

1. Flow visualization of the flowfleld using neutrally-buoyant helium-filled soap bubbles, and mult1-sparks.

2. Detailed hot-wire measurements of timemean velocities, three normal and three shear turbulent stresses using the sixorientation single-wire hot-wire technique.

\subsection{Previous Studies}

Previous research conducted at Oklahoma State University on combustor-type flowfields without lateral injection jets are summarized by Lilley.10,11 They included experimental and theoretical research under low speed, nonreacting, turbulent, swirling flow conditions. The flow enters the test section and preceeds into a larger chamber (the expansion $\mathrm{D} / \mathrm{d}=2,1.5$ or 1) $\mathrm{via} a$ sudden or gradual expansion (slde-wall angle $\alpha=90$ and 45 degrees). A weak or strong nozzle may be positioned downstream to form a contraction exit to the test section. Inlet swirl vanes are adjustable to a variety of vane angles with values of $\phi=0$ (swirler removed), 38, 45, 60 and 70 degrees being emphasized. The objective was to determine the effect of these parameters on isothermal flowfield patterns, time-mean velocities and turbulence quantities, and to establish an improved simulation In the form of a computer prediction code equipped with a sultable turbulence model. Hellum bubble flow visualization, five-hole pitot probe time-mean velocity measurements, and one-wire and two-wire not-wire normal and shear stress turbulence data were obtained in the experimental program. Turbulence modeling deductions and flowfleld predictions were made $v i a$ a 2-D axisymmetric technique.

An outgrowth of the above research is the research program at Oklahoma State University which deals both experimentally and theoretically with the problem of primary and dilution lateral jet injection into typical combustor flowfields in the absence of combustion. Parameter variations to be systematically investigated include: lateral jet velocity, number and location of lateral jets, combustor crossflow inlet swirl strength, and downstream contraction nozzle location and strength. The general goal is to characterize the time-mean and turbulence flowfield with a varlety of parameter settings, recommend appropriate turbulence model advances, and implement and exhibit results of flowfleld predictions.

Significant earller studies elsewhere include Chassaing et $a,^{12}$ Rathgeber and Becker, ${ }^{13}$ Crabb et a1 ${ }^{14}$ and Holdeman et al.1" The recent $\mathrm{Ph}$. D. thesis by Ferrel1 ${ }^{3}$ provides an extensive review of these and other studies on the lateral jet injected into crossflow. Ferrell completed his detalled work on the single lateral injected jet with velocity ratio $R=v_{j} / u_{0}=2,4$ and 6 entering into nonswirling crossflow, using the six-orientation single hot-wire measurement technique. In addition to this, several flow visualization techniques (bubbles, smoke and sparks) were used with these same velocity ratios into swirling crossflow, with swirl vane angle $\phi=0$ (swirler removed), 45 and 70 degrees. Detalled measurements were not made for these swirling cases. Conference papers were prepared by Ferrell and colleagues dealing with preliminary results and computer simulation, ${ }^{6}$ flow visualization, ${ }^{7}$ and detalled hot-wire measurements. ${ }^{3}$

The objective of current research is to emphasize the swirl effect on lateral injection into tubular crossflow. A concurrent research effort on the single lateral jet with $R=4$ entering the 0,45 and 70 degree swirling crossflow is being undertaken by Ong. 9 The present study is highlighted by two opposed lateral jets with $R=4$ entering the 0,45 and 70 degree swirling crossflow.

\subsection{Outlline of the Paper}

Section 2 describes the experimental facility, test-section, and dilution jets. The data acquisition system is briefly described and other equipment used in the investigation is mentloned. Flow visualization and measurement technlques are discussed in Section 3. Results of the flow visualization and measurement techniques are discussed thoroughly in Section 4. Section 5 presents the conclusions to be drawn from this investigation.

\section{EXPERIMENTAL FACILITY}

\subsection{Hind Tunnel And Vane Suirler}

The experimental faclitity is the same as used on the previous experimental program without lateral jets and described at length in previous $M$. $S$. and $\mathrm{Ph}$. D. theses and in abbreviated form in several conference papers included in Ref. 10. A complete description of the wind tunnel, nozzle and facility layout is given by Ferrell." The test facility is shown schematically in Figure 1 . It consists of a wind tunnel, a varlable-angle vane swirler, and a plexiglass test section of diameter $15 \mathrm{~cm}$. The wind tunnel has a contraction section whose contour conforms to the method of design suggested by Morel ${ }^{16}$ to produce a minimum adverse pressure gradient on the boundary layer and thus avold unsteady problems assoclated with local separation regions. The air-flow passes through a $15 \mathrm{~cm}$ dlameter circular jet nozzle, exiting into a $15 \mathrm{~cm}$ diameter test section of length $90 \mathrm{~cm}$, which is constructed of plexiglass to facilitate flow visualization.

A variable-angle vane swirler may be positioned Immediately before the test section. Its flow area has an outer diameter of $15 \mathrm{~cm}$. The swirler has ten vanes which are individualiy adjustable to any angle $\phi$ and $a$ hub with $a$ streamlined upstream nose and a flat downstream face. The nose has a hyperbollc shape with a very smooth surface so as to offer minimal flow interference. The flat blades are wedge-shaped to give a constant pitch-to-chord ratio of 0.68 which gives good turning efficlency. ${ }^{3}$ Its performance is documented elsewhere. ${ }^{10}$ The test section begins at a location $x / D=0$, which is $3.2 \mathrm{~cm}$ downstream of the swirler exit.

\subsection{Test Section and Dilution Jets}

Figure 1 also shows the configuration of the $15 \mathrm{~cm}$ diameter test section (the same as the exit throat) mounted on the wind tunnel with the swirler omitted. Air to the lateral dilution jets is supplied from a compressed alr IIne via plping and carefully-designed nozzles. Upstream of each nozzle, a stagnation chamber, turbulence management 


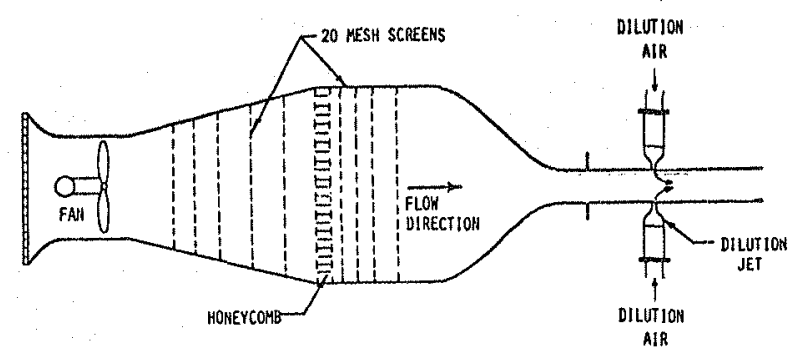

Fig. 1. Schematic of Experimental Facility screens, flow straightening straws, and flow metering equipment are used for flow conditioning. Experiments have been performed on the present study with the two opposed lateral jets located one test-section diameter downstream of the inlet.

The test section consists of a clear acrylic tube approximately $90 \mathrm{~cm}$ in length attached to the wind tunnel throat. Standard commercial acrylic tube is used with $15.24 \mathrm{~cm}(6.0$ in.) outside diameter, $0.318 \mathrm{~cm}(0.125 \mathrm{in.})$ wall thickness. The inside diameter is $14.61 \mathrm{~cm}$ with a measured variation of $\pm 0.05 \mathrm{~cm}$. To adapt the test section to the wind tunnel throat (inside diameter $15 \mathrm{~cm}$ ), an adaptor section was machined to provide a smooth transition from the wind tunnel throat to the test section.

The test section tube allows probe access to locations downstream of $x / D=1.75,2.00,2.50$, 3.00 and at any azimuthal angle. This is accomplished via a tube rotation section, constructed from machined aluminum rings, acrylic, and ball bearings as seen in Figure 2. A photograph showing both jets in position on the test section is seen in Figure 3. Also visible is the valve arrangement for five-hole pitot probe measurements.

A more complete description of the dilution jets and air supply appears in Reference 5 .

\subsection{Equipment}

Equipment used in this investigation includes the calibration jet, data acquisition and probe drive system, DISA type 55P01 normal hotwire probe, DISA 55M21 probe support, DISA 55M01 anemometer, Burr-Brown SDM853 12-bit A/D converter, and an Apple IIe computer. These are the same as used previously and described in full in Reference 5 .

\section{INVESTIGATION TECHNIQUES}

\subsection{Bubble Flow Visualization}

Flow visualization ${ }^{4}, 17$ is used primarily for the identification and characterization of the flowfield, with two techniques being used in the present study: bubbles (described in the present section) and sparks (described in the next section). The equipment and techniques are described fully by Ferrell and colleagues ${ }^{5,7}$ in regard to their use on the present facility.

Bubbles, because of their reflective qualities and neutral buoyancy in the airflow, provide an excellent medium to determine the paths of the jet trajectories. The technique has been used on investigations without lateral jet injection 10,11 and on the single lateral jet injection. ${ }^{5,7}$ A bubble generator, manufactured by Sage Action, Inc. is used to supply up to 100 bubbles per second, and inject them into the lower lateral jet.

The lighting for bubble flow visualization consists of light from a high-intensity slide projector located approximately $3 \mathrm{~m}$ downstream of the test section. A slit in an opaque slide provides a vertical light curtain about $1.5 \mathrm{~cm}$ wide. The lighting is on throughout the photography session and the exposure time of

Fig. 3. Dilution Jets Mounted on Test Section. 
typically 5 seconds permits streamlines to be identified. The camera used is a Minolta SRI 200. The films used include Kodak Tri-X Pann 400 ASA black and white, Ilford 400 ASA black and white, and Kodak color 1000 ASA film, with all of these giving excellent results. The camera is positioned approximately $0.5 \mathrm{~m}$ laterally from the test section and supported by a tripod. A low Fstop of 2 is used for maximum light intake, to accentuate the bubble streaklines illustrating the flow trajectories.

\subsection{Spark Flow Visualization}

A multi-spark visualization method has also been used on the present study. ${ }^{4,5,7}$ The technique uses an ionized path between two electrodes. This path moves with the air flow, and is sequentially lit up by successive sparks. The pulse generating circuit and pulse transformer are manufactured by Sugawara Laboratories, Inc., Tokyo. Equipment specifications and operating procedures are given in References 5 and 7.

When a high voltage source is sparked across an air gap, an ionized path is created. Subsequent sparks will follow the current position of this low-resistance ionized path. By placing electrodes in the wall boundary layer, where there is essentially zero velocity (next to the wall), several discharges can follow the ionized path as it moves with the fluid. The test section materials must have low electrical conductivity such as acrylic so as not to interfere with spark paths. The spark itself provides sufficient lighting for photographs. One camera (side view) is used for photographs with zero swirl. Two cameras (side and end view) are used simultaneously in the swirl crossflow cases to give added perspective to the three-dimensional features of the resulting flowfield.

\subsection{Hot-Wire Anemometry}

In a turbulent, three-dimensional flowfield the main flow direction may be unknown and conventional hot-wire or Laser-Doppler techniques fail to supply sufficient velocity vector information. To measure the three velocity components and their corresponding fluctuations, a three-wire hot-wire probe is often used. Few 3-D Laser-Doppler systems are in use and are not costeffective. The three-wire probe technique has several drawbacks. Three anemometers are required. A multiple-orientation probe drive may be needed to align the probe with the mean flow direction. Because of the physical separation of the wires, spatial resolution of the probe is poor. A recent textbook surveys the experimental challenges and assesses available techniques. ${ }^{4}$

Multi-orientation of a single not-wire is a novel way to measure the three components of a velocity vector and their fluctuating components. In the present study, the six-orientation singlewire hot-wire method is used exclusively for detailed measurements. It is described in the M.S. and Ph.D. theses of Janjua ${ }^{18}$ and Jackson. ${ }^{19}$ This method calls for a normal hot wire to be oriented through six different positions, each orientation separated by 30 degrees from the adjacent one. Orientation 1 is normal to the facility centerline, orientation 2 is rotated 30 degrees from this, etc. Time-mean and root-mean-square voltages are measured at each orientation. The data reduction is performed using several simplifying assumptions regarding the statistical nature of turbulence, making it possible to solve for three time-mean velocities, the three turbulent normal stresses, and the three turbulent shear stresses.

The six-orientation hot-wire technique requires a single, straight, hot wire to be calibrated for three different flow directions in order to determine the directional sensitivity of the probe. When the wire is placed in a threedimensional flowfield, the effective cooling velocity experienced by the not wire may be deduced from the calibration curves. Hence, equations for the effective cooling velocity can be obtained for each of the six wire orientations. Simultaneously solving any three adjacent equations provides expressions for the instantaneous values of the three velocity components ( $u, v$, and $w$ in the facility $x, r$, and $\theta$ coordinates, respectively) in terms of the equivalent cooling velocities. It is then possible to obtain the three time-mean velocity components and the six different components of the Reynolds stress tensor in the manner described by Janjua ${ }^{18}$ and Jackson, ${ }^{19}$ which is described briefly in research papers included in Ref. 10. Jackson also assessed the accuracy and directional sensitivity of the technique, concluding that severe inaccuracles may be present, especially in the deduced shear stress values which are discussed in Section 4.2. Jackson found that the configuration of probes versus local flow direction is of little importance. He recommended evaluating quantities from the average of all six possible wire combinations, and this smoothing has been used exclusively in recent studies and in the present study.

\section{RESULTS AND DISCUSSION}

\subsection{Flow Visualization}

Neutrally-buoyant helium filled soap bubbles injected through the lower jet, located at $x / D=$ 1.0, trace pathlines clearly when illuminated. Figure 4 presents time exposures of bubble pathlines for the case of 2 jets, $R=4, \phi=0$ (swirler removed), 45 , and 70 degrees in parts $a$, $b$, and $c$ respectively. Vertical slit lighting is used as discussed in section 3.1 to obtain a view of the rx-plane.

Part a of Eigure 4 reveals the depth of penetration of the dilution jet. The jet does not cross the centerline of the test section because of the symmetry effect of the presence of the opposing dilution jet. Ferrell ${ }^{5}$ found for the case of a single lateral jet with the same velocity ratio $\mathrm{R}=$ 4 that the jet penetrates the test-section centerline at approximately $x / D=1.4$. In the swirl flow case, parts $b$ and $c$ of Figure 4 , the extent of jet penetration is not readily discernable. In the case of $\phi=45$ degrees, the helical path of the jet is visible. The region from the dilution jet inlet $x / D=1.00$ to approximately $x / D=2.00$ indicates, by lack of visible bubbles that the jets do not immediately mix with the precessing vortex core (PVC). The jet trajectory has spiraled away from the light curtain. The presence of the opposed jets appears to delay the onset of mixing with the PVC as compared to the case of one dilution jet. ${ }^{5}$ In the case of $\phi=70$ degrees, the centrally-located PVC 


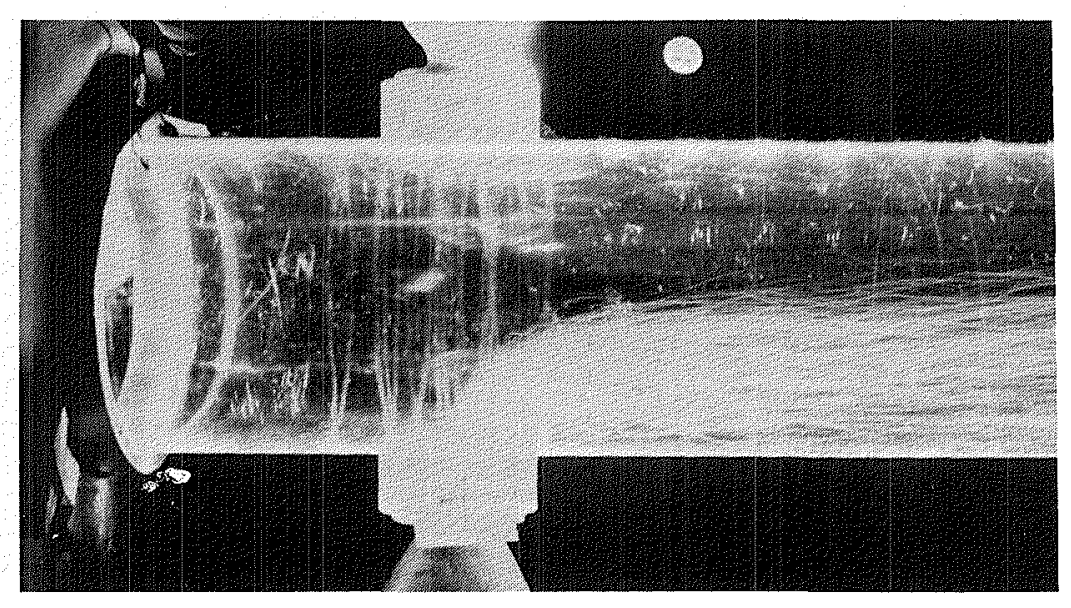

(a) Swirl Vane Angle $\phi=0$ Degrees (Swirler Removed)

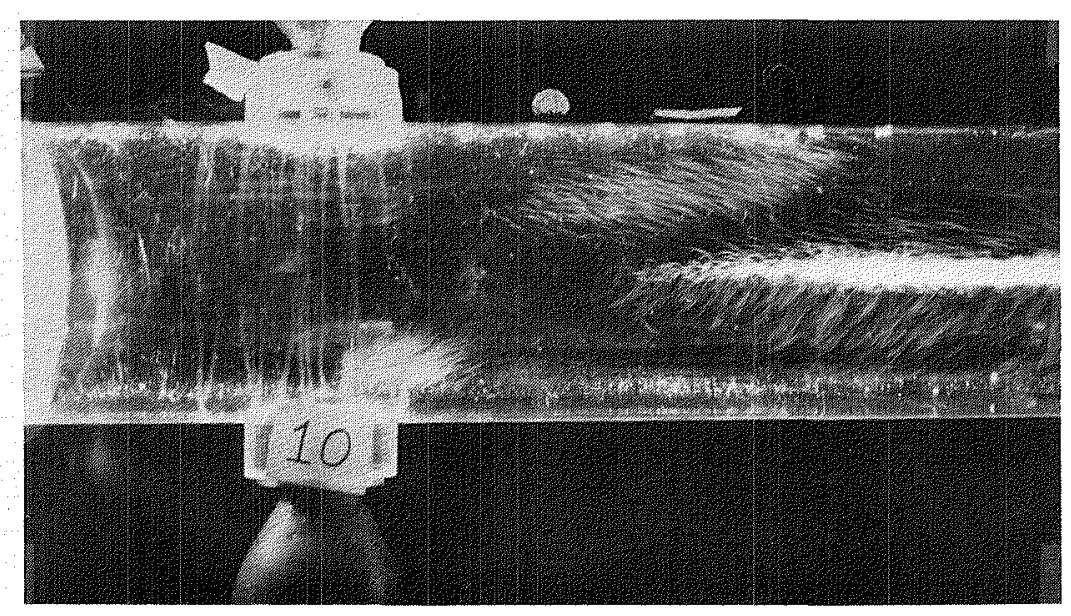

(b) Swirl Vane Angle $\phi=45$ Degrees

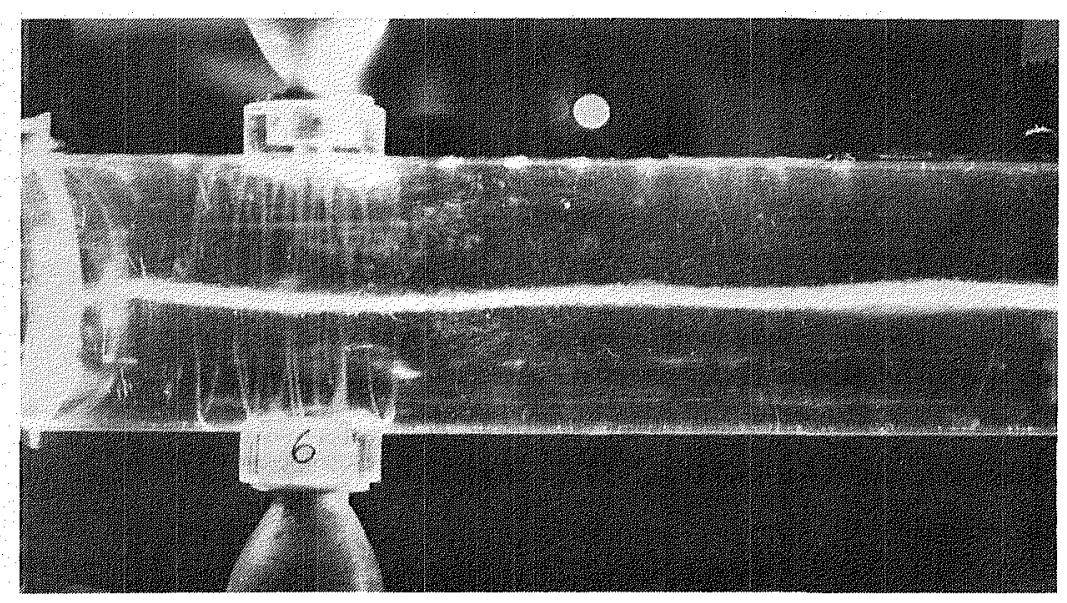

(c) Swirl Vane Angle $\phi=70$ Degrees

Fig. 4. Bubble Flow Visualization 


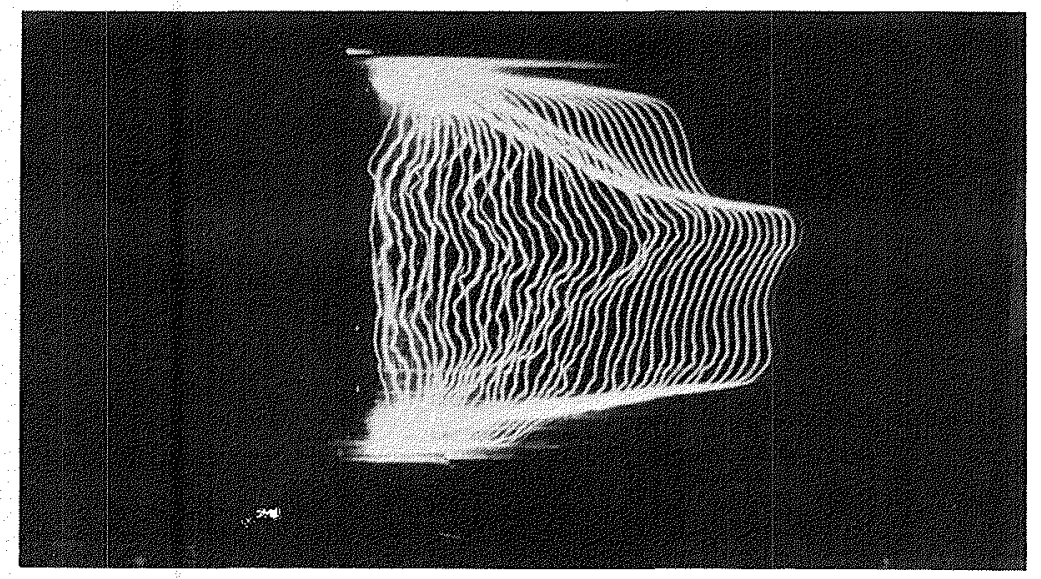

(a) Swirl Vane Angle $\phi=0$ Degrees (Swirler Removed)

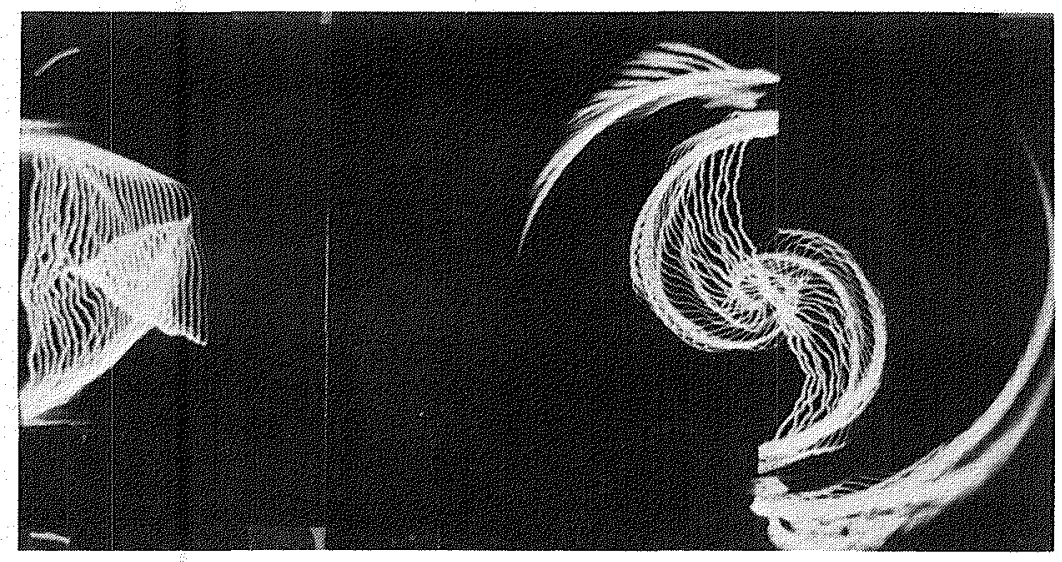

(b) Swirl Vane Angle $\phi=45$ Degrees

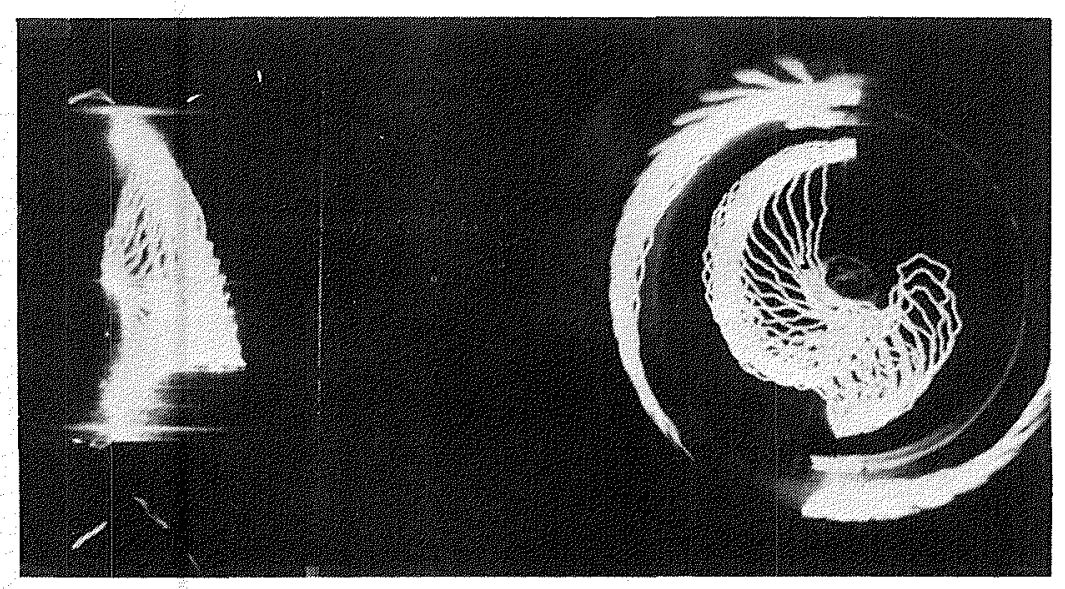

(c) Swirl Vane Angle $\phi=70$ Degrees

Fig. 5. Spark Flow Visualization 
occuples a much narrow region in the opposed jet case, as compared with the single and no lateral infection jet cases.19,20 The flow centerline is slightly deflected from the facility centerline throughout the test section, appearing to oscillate about the centerline for the strong swirl case.

Figure 5, parts $a, b$, and $c$ presents multispark flow visualization pictures for the $R=$ 4 jet entering different crossflow swirl conditions with $\phi=0$ (swirler removed), 45 and 70 degrees of swirl, respectively. Electrodes are positioned at $x / D=1.5$. In the M.S. thesis of McMurry, ${ }^{21}$ the electrodes are positioned at $x / D=0.5,1.5,2.0$, and 2.5 to identify changes in the flowfield with movement downstream. The opposed jets are located at $\mathrm{x} / \mathrm{D}=1$.

In part $a$, the camera is positioned to the side of the test section to obtain a view of the rx-plane. The influence of the opposed jets is clearly visible. Near the center of the test section, the flow is accelerated as indicated by the larger spacing between the sparks near the center of the section. Near the top and bottom of the test section, the presence of the opposed jets is indicated by the sparks wrapping around the boundaries of the jets. With distance downstream the opposed jets were found to amalgamate with the crossflow to exhiblt the characteristic flat velocity proflle of turbulent flows in round tubes.

In parts $b$ and $c$ of Figure 5, which include the effects of swirl, a second camera was positioned downstream and simultaneously operated to obtain the ro-plane behavior. The rx-plane and the $r \theta-p l a n e$ photographs were then combined to form a common plcture of the flow behavior at various $x / D$ locations. A wire was placed at the centerline of the test section to prevent the spark from areing to the tube wall and to help define the center of the tube. The end views show a considerable reflection of the spark of $f$ the test section wall.

Flgure $5 b$ is for the case of swirl vane angle $\phi=45$ degrees. With swirl vane angle $\phi=45$ degrees, little varlation in the swirl pattern is distinguishable up to $x / D=2.5$. At $x / D=2.5$ however, there appears to be an elongation of successive sparks, and a strengthening of the centrally located PVC. The apparent strengthening of the PVC corresponds well with the appearance of bubbles in the bubble-flow visualization pictures of Figure 4b. Figure $5 \mathrm{c}$ presents multi-spark photography for the case of $\phi=70$ degrees. Interpreting the flowfield is difflcult due to the spark pattern. The spark path appears to have arced past the wire at the center of the test section. It does not exhibit the symmetric swirl pattern of locations further downstream, ${ }^{21}$ where the symmetrical spark shows no direct influence from the opposed jets such as being deflected around the jets.

\subsection{Hot-Hire Measurements}

Figures 6 through 23 present the time mean velocity components for jet-to-crossflow velocity ratio $R=4$, swirl vane angle $\phi=0$ (swirler removed), 45, and 70 degrees. Flgures 6 through 11 have a swirl vane angle $\phi=0$ (swirler removed) with a jet-to-crossflow velocity ratio of $R=4$. Figure 6 has a traverse angle $\theta=0$ degrees (swirler removed), Figure 7 has a traverge angle $\theta=330$ degrees, etc. Figures 12 through 17 have $R=4$ with swirl angle $\phi=45$ degrees. Figures 18 through 23 present the case of $R=4$, swirl angle $\phi=70$ degrees. The geometrical relationship of the traverse angle to the test section may be seen in Figure 2. For the traverse angle $\theta=0$ degrees, the vlewer is seeing a vertical rx-plane of the flowfield which passes through the test-section centerline and through the opposed jet nozzles, as in Figure 6 for example.

In each of the Figures 6 through 23, subparts $a, b$, and $c$ present normalized time-mean velocity components. In the M.S, thesis of MaMurry, ${ }^{21}$ the normal and shear stresses are also presented. As mentioned prevlously, the accuracy of results for turbulence quantities (normal stresses and especlally shear stresses) is in doubt. Jackson's discussed the quality of the hot-wire technique in a weakly swirling axisymmetric expanded flowfleld with $\mathrm{D} / \mathrm{d}=2$. He found that in turbulent shear reglons, the maximum errors are $18,24,29$, and 98 percent for time-mean values, normal stresses, shear stresses and $\overline{u^{\prime} w^{\prime}}$, respectively. The present study emphasizes the stronger swirl without expansion into the test section and may have potentlally further accuracy problems. One major limitation with the present data acquisition system is the limited sampling rate of $1000 \mathrm{~Hz}$. Higher frequency signals are not properly digitized due to an aliasing effect. Signal frequencies above 1000 $\mathrm{Hz}$ should be filtered out in the data acquisition system. However, the data acquisition system used in this study does not include the use of a filter. Thus, the data includes any allasing of high frequency signals. The amalgamated effect of these uncertainties leads to an unknown degree of error in the calculated values of turbulence quantitles, especially the shear stresses. There are no alternative sources of data with which to compare the deduced values.

The plots were produced on a Tektronix 4006 terminal connected to an IBM $3081 \mathrm{D}$ using PLOT 10 as the graphics control language. The data are merely scaled and plotted point-to-point for each axial location. The $x / D$ scales also provide as the magnitude scale for each normalized data point. For example, in Figure $6 a$, the values of $\bar{u} / u$ at $x / D=1.75$ are scaled such that values of $\bar{u} / \mathrm{u}_{0}=$ 1.0 are placed at $x / D=2.00$.

The case of jet-to-crossflow velocity ratio $R$ $=4$ and swirl vane angle $\phi=0$ degrees (swirler removed) is presented in Figures 6 through 11. Figure $6 a$ presents the axial velocity profiles through the center of both jets. At $x / D=1.75$ it appears the opposed jets have already met at the centerline of the test-section as evidenced by the higher velocities at the centerline. At $x / D=$ 2.00 , the jets are mixing with the crossflow tending to decrease the centerline velocity. At $x / D=2.5$, the velocity profile is uniform throughout the test section. Figure 9 provides a view of the flowfield normal to the entrance direction of the opposed jets. At $x / D=1.75$, part a shows the jets to have met near the centerline. At $x / D=2.00$, the crossflow appears to have accelerated around the sides of the jet, due to the obstruction to flow caused by the jets. With distance downstream, the velocity profiles begin to flatten out.

The case of jet-to-crossflow velocity ratio 

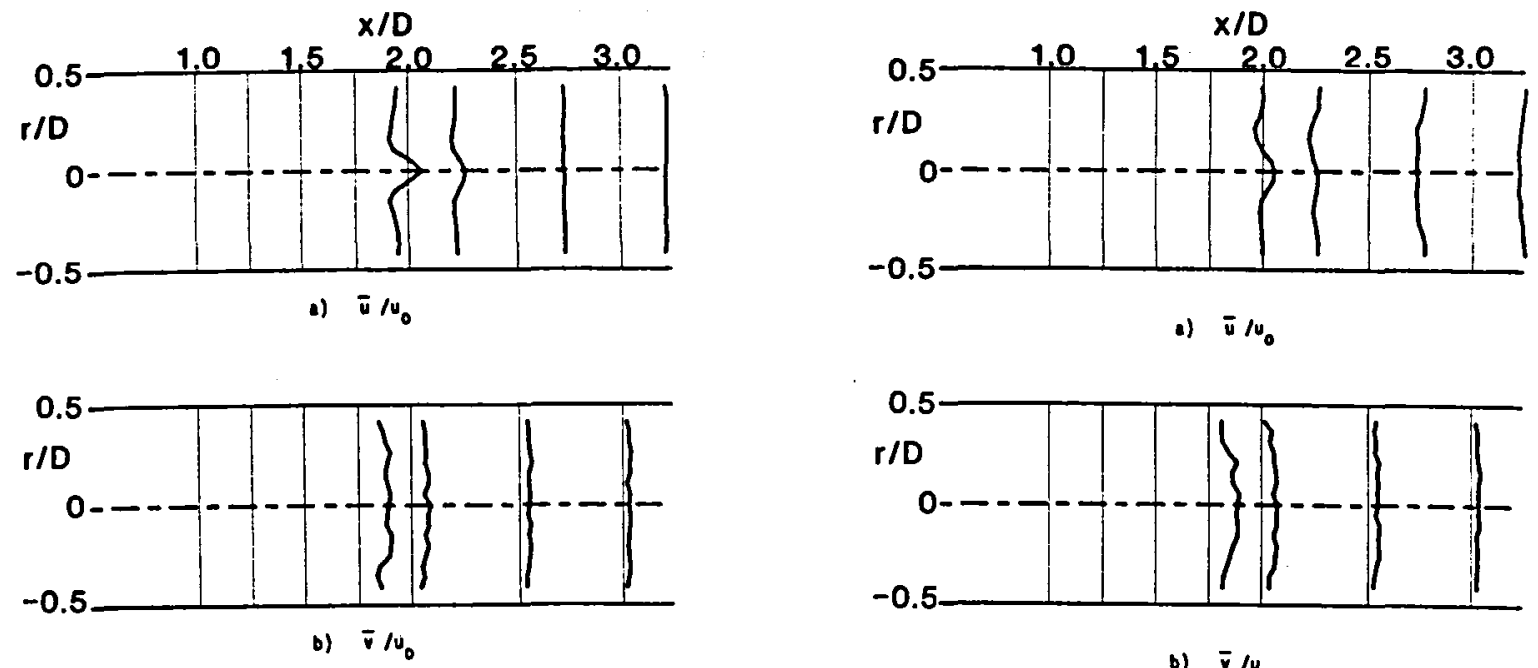

b) $\bar{v} / u_{0}$
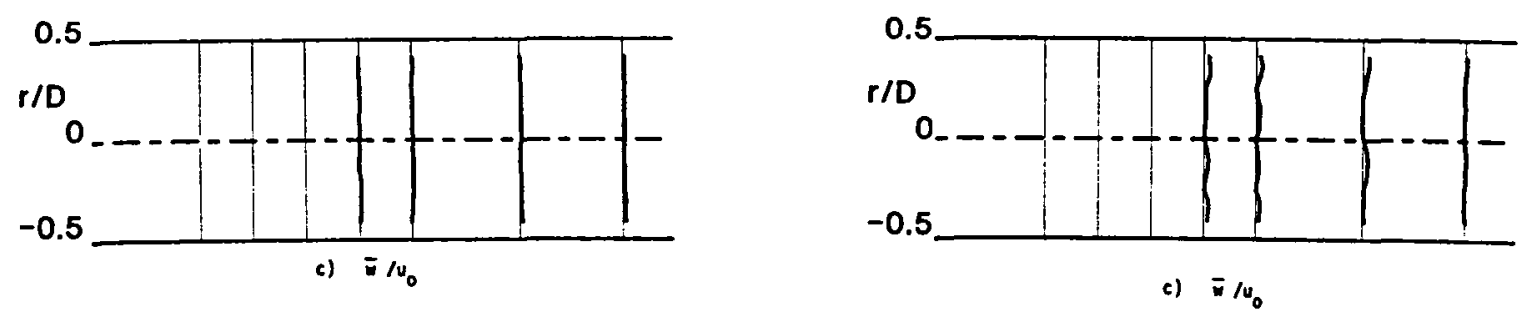

Fig. 6. Time-Mean Velocity with $\phi=0$ Degrees (Sw1rler Removed)

Traverse Angle $\theta=0$ Degrees.

Fig. 8. Time-Mean Velocity with $\phi=0$ Degrees (Swirler Removed)

Traverse Angle $\theta=300$ Degrees.

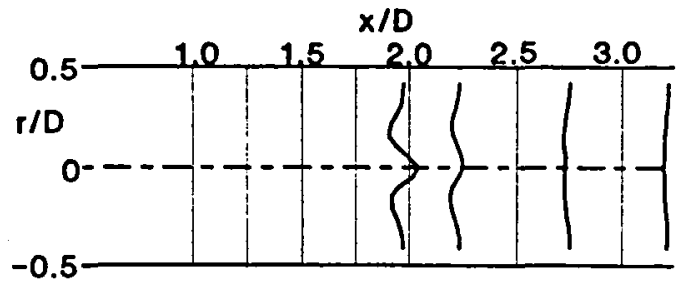

a) $\bar{u} / 0$

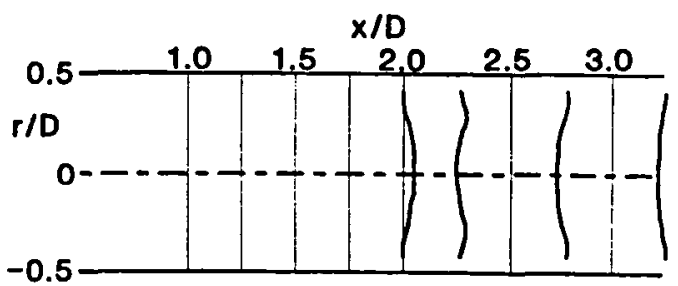

.) $\overline{1} / 0_{0}$

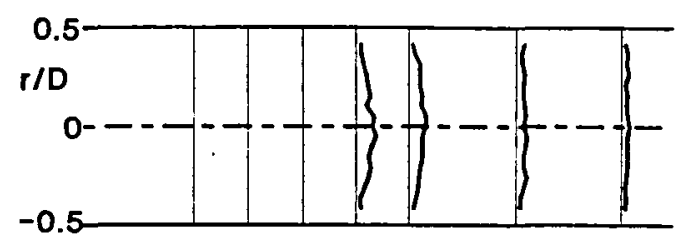

b) $\bar{v} / 0$

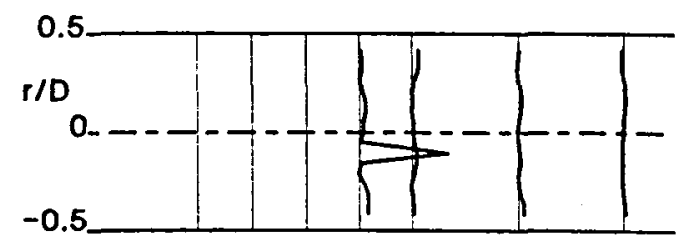

c) $\overline{1} / \mathrm{u}_{0}$

Fig. 7. Time-Mean Velocity with $\phi=0$ Degrees (Swirler Removed)

Traverse Angle $\theta=330$ Degrees.

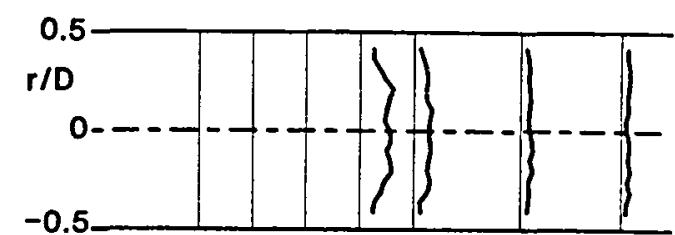

b) $\bar{v} / 0$

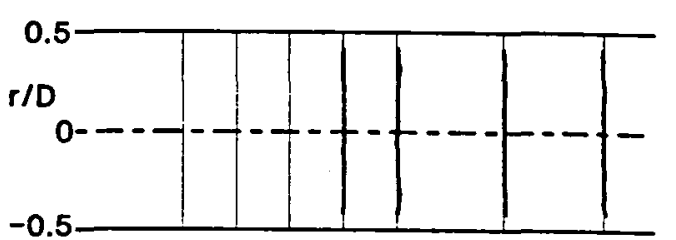

c) $=10$

Fig. 9. Time-Mean Velocity with $\phi=0$ Degrees (Swirler Removed)

Traverse Angle $\theta=270$ Degrees. 


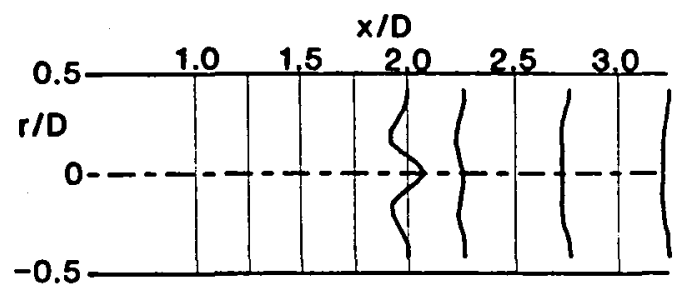

a) $\bar{u} / u_{0}$

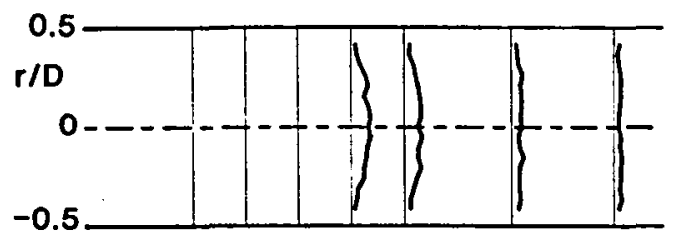

b) $\bar{v} \omega_{0}$

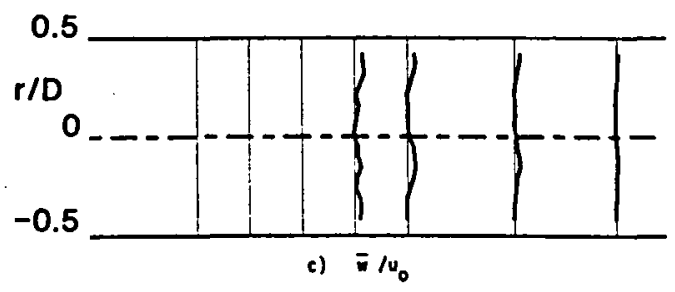

Fig. 10.Time-Mean Velocity with $\phi=0$ Degrees (Swirler Removed)

Traverse Angle $\theta=60$ Degrees.

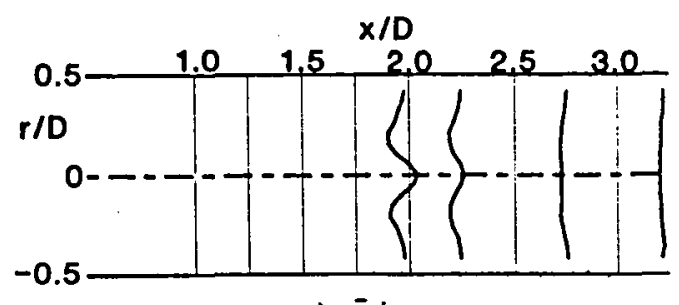

.) $\bar{u} / 0$
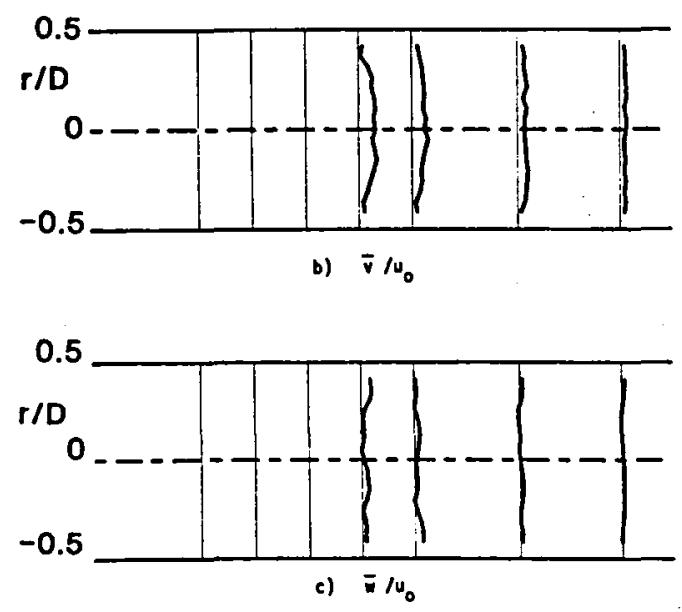

Fig. 11.Time-Mean Velocity with $\phi=0$ Degrees (Swirler Removed)

Traverse Angle $\theta=30$ Degrees.
$R=4$ and swirl angle $\phi=45$ degrees is presented In Flgures 12 through 17. Figure 12 presents a vertical $r x$-plane view of the flowfield, passing through the entrance of the opposed jets. There is evidence of a precessing vortex core (PVC) as indicated in Figure 12, parts $a$ and $c$, by the low axial velocity and almost solid body rotation near the axis at the center of the test section. The upstream velocity of the crossflow is measured before the crossflow reaches the swirler. Thus, after introduction into the swirler, the net crossflow velocity experienced by the opposed jets is larger due to the swirl component of the total crossflow velocity. Thus, the true jet-tocrossflow velocity ratio is lower, which will reduce the penetration of the opposed jets into the crossflow.s For the 45 degree swirl case, the $R$ value is reduced to approximately 2.8 .

The angle $\delta$ between the local crossflow direction and the plane normal to the facility centerline is calculated by $\delta=\arctan (u / w)$ locally. Near the injection locations of the lateral jets, this angle is approximately $\delta=45$ degrees for the moderate swirl case $(\phi=45$ degrees). For the strong swirl case $(\phi=70$ degrees) the angle $\delta$ is approximately 27 degrees. A spiralling path on the tube wall would require a normalized downstream distance of $L / D=\pi(\tan \delta)$ to complete one revolution of the tube. The trajectory of the lateral jets follow roughly this path, but the angle $\delta$ changes as the jet penetrates into the field. Assuming the angle $\delta$ remains constant, for the moderate swirl case of $\phi=45$ degrees ( $\delta=$ 45 degrees), the required normallzed downstream distance is $L / D=3.14$; for the $\phi=70$ degree case $(\delta=26$ degrees $), L / D=1.5$. Hence, evidence of the lateral jets are expected to be seen at $x / D=$ 1.75 in the $\theta=270$ degree traverse, etc. for the moderate swirl case. For the strong swirl case, it is expected to be seen at $x / D=1.75$ in the $\theta=0$ degree traverse, at $x / D=2.0$ in the $\theta=300$ degree traverse, etc.

The clearance requirement of the probe-drive system does not allow measurements to be taken upstream of $x / D=1.75$ so tracking of initial jet trajectories is not possible. However, the presence of the opposed jets is visible in Figure 15, $\theta=270$ degrees. The lateral jets are swept along with the swirling crossflow. The two axtal velocity maxima indicating the presence of the jets at $x / D=1.75$ are not visible at the other downstream locations. The jets may be traced further downstream by viewing Figure $13 \quad(\theta=330$ degrees), part a at $x / D=2.00$. Similar velocity maxima are observable in Figure $14 a(\theta=300$ degrees) at $x / D=2.00$ but at a lower axial velocity. It appears that the traverse of Figure $13 a$ at $x / D=2.00$ came near the center of the jets while the traverse of Figure 14 a merely passed through the edge of the jet boundaries. The effect of the opposed jets on the velocity profiles may be seen in Figures 12 through 17 . Comparing part a of these figures, the axis of symmetry for the timemean velocities appears to shift around the centerline of the test section.

In the strong swirl case, $\phi=70$ degrees, the angle between the local crossflow direction and the plane normal to the facility centerline, $\delta=$ arctan $(u / w)=27$ degrees near the lateral jet injection locations, as discussed previously. This requires approximately a downstream distance of $L / D=1.5$ 

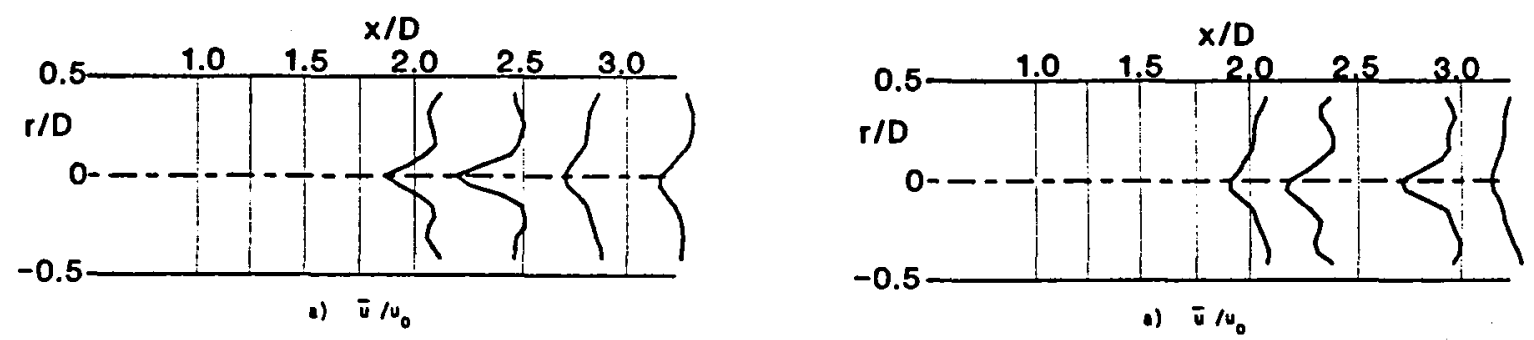

1) $\bar{v} / u_{0}$
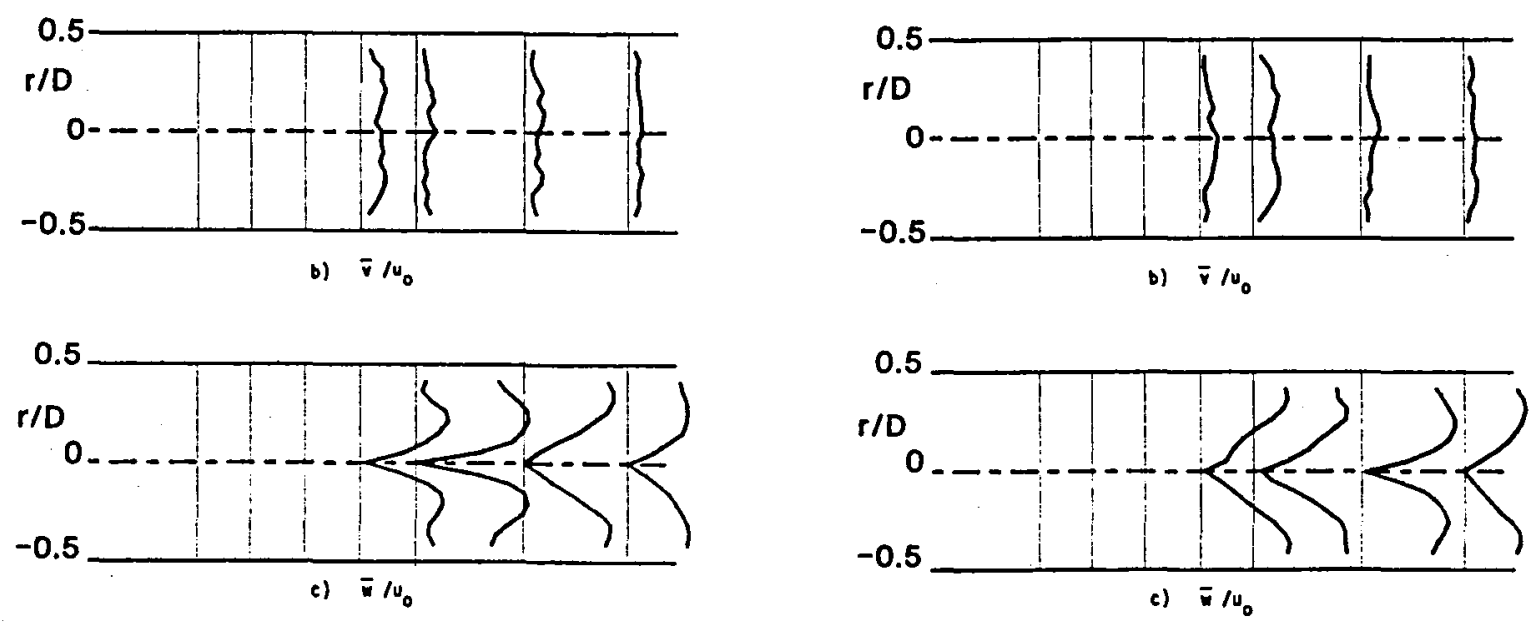

F1g. 12.Time-Mean Velocity with $\phi=45$ Degrees, Traverse Angle $\theta=0$ Degrees.

Fig. 14.Time-Mean Velocity with $\phi=45$ Degrees, Traverse Angle $\theta=300$ Degrees.
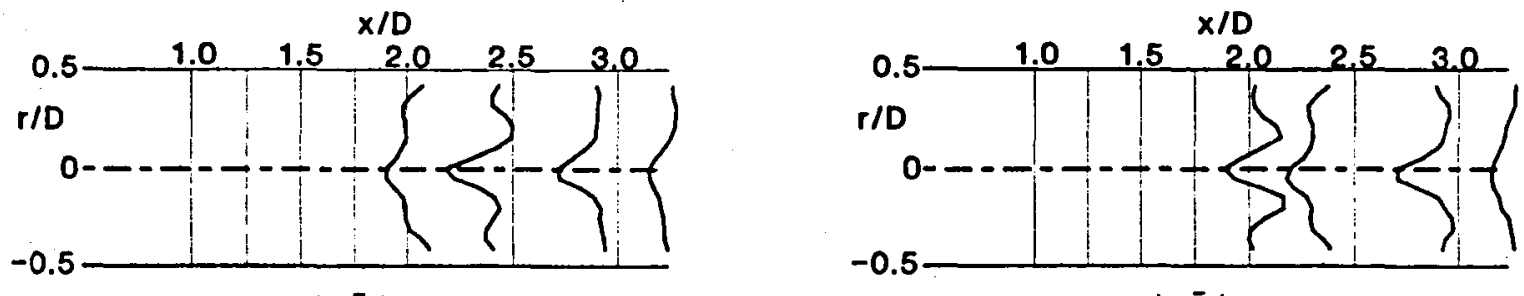

.) $\bar{u} / \omega_{0}$
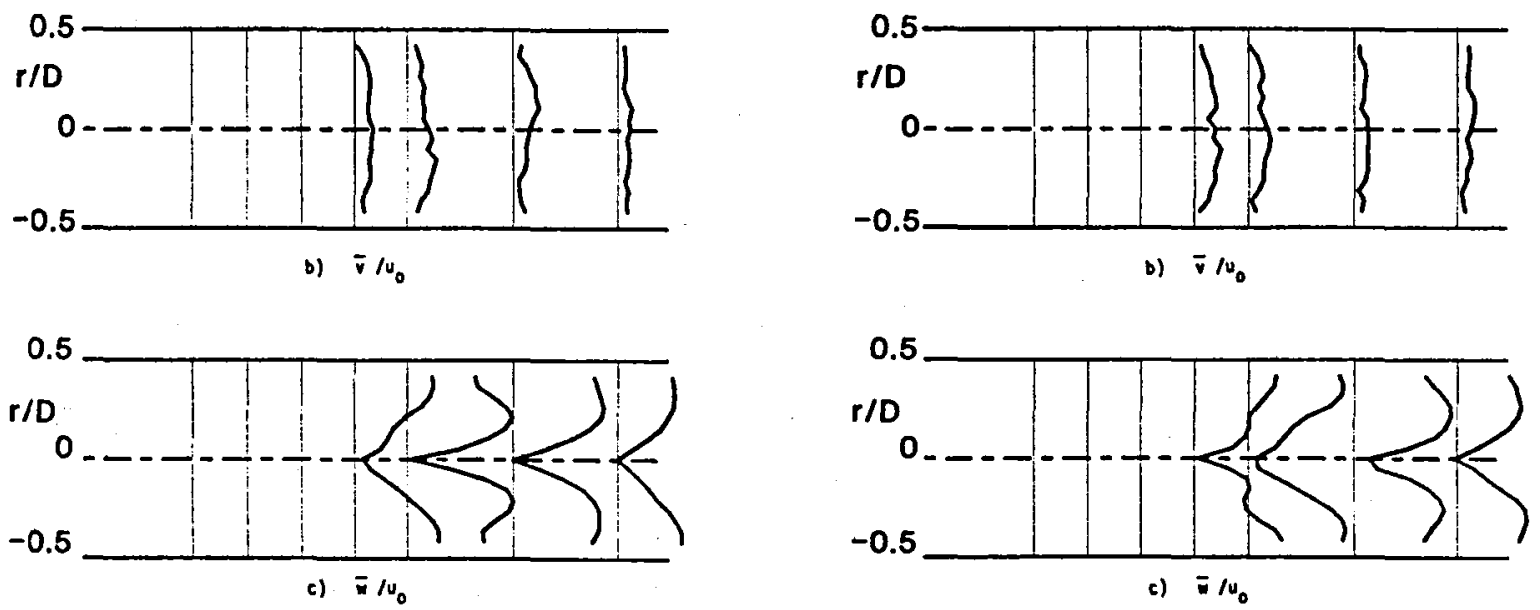

Fig. 13.Time-Mean Velocity with $\phi=45$ Degrees, Traverse Angle $\theta=330$ Degrees.

F1g. 15. Time-Mean Velocity with $\phi=45$ Degrees, Traverse Angle $\theta=270$ Degrees. 

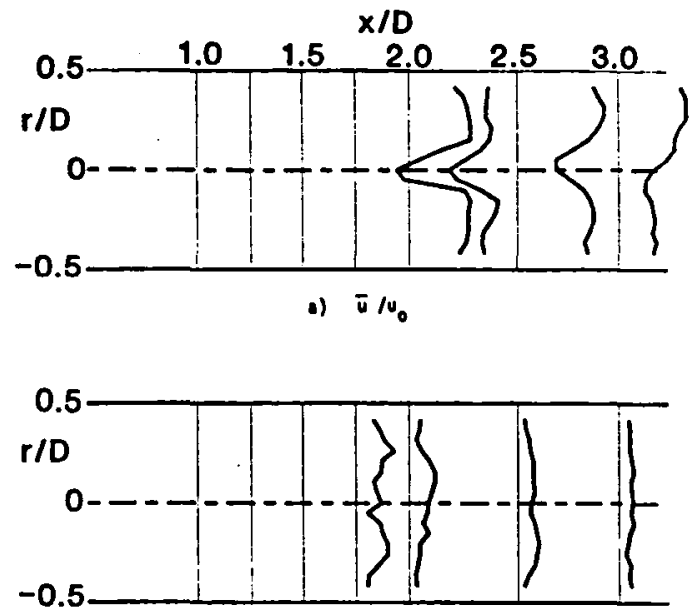

b) $\bar{v} / 0$

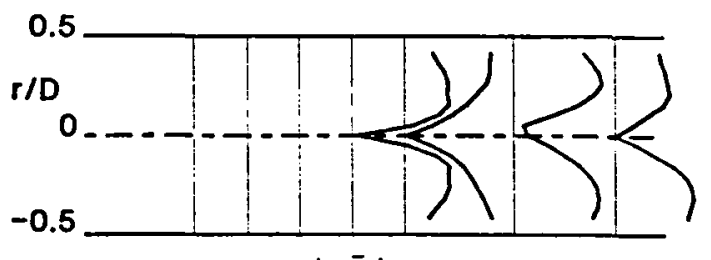

c) $\overline{1} / 0$

Fig. 16.Time-Mean Velocity with $\phi=45$ Degrees, Traverse Angle $\theta=60$ Degrees.
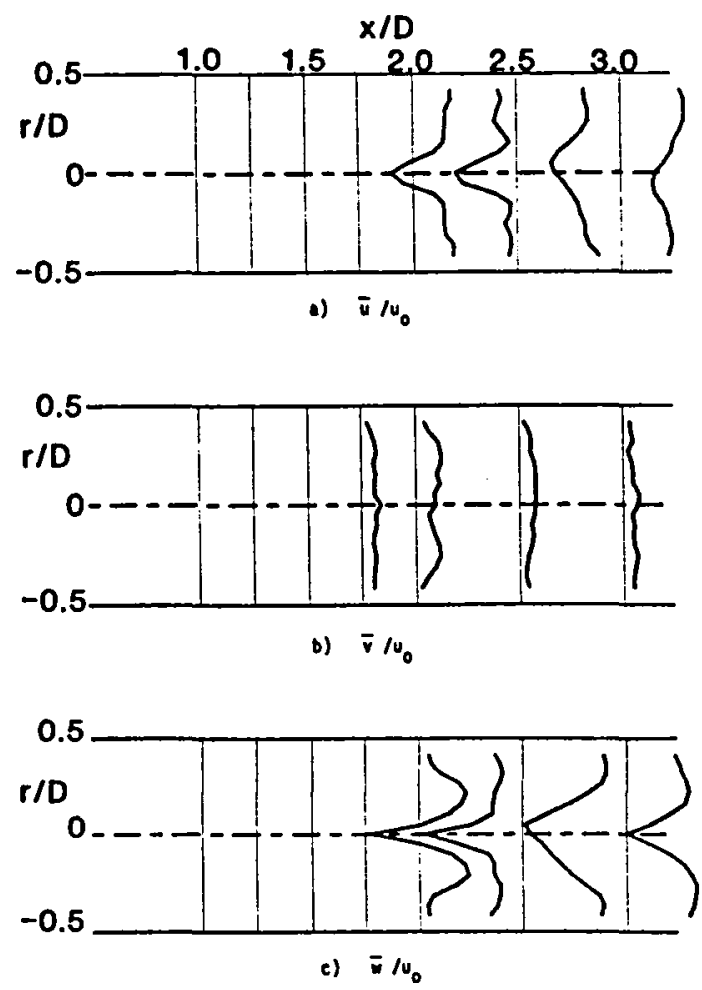

F1g. 17.Time-Mean Velocity with $\phi=45$ Degrees, Traverse Angle $\theta=30$ Degrees. for the path of the jet to ideally make one revolution, with evidence of the lateral jet expected to be seen at $x / D=1.75$ in the $\theta=0$ degree traverse, at $x / D=2.00$ in the $\theta=300$ degree traverse, etc. Time-mean velocity profiles are presented in Figures 18 through 23 for the case of jet-to-crossflow velocity ratio $R=4$, swirl vane angle $\phi=70$ degrees. Part a of the flgures show that most of the flow is confined to the region near the wall due to high centrifugal forces. The reduced jet-to-crossflow velocity ratio, because of the swirling velocity component in the crossflow, results in the true jet-tocrossflow ratio $\mathrm{R}$ at approximately 1.2. Previous studies ${ }^{5}$ show that jet penetration into the crossflow will be substantially reduced. Flow visualization photographs indicate that this is indeed the case. Figures 18 through 23 show no direct evidence of the jets. However, as noted in the case of $\phi=45$ degrees, thelr effect on the symmetry of the velocity profiles is indicated by comparing the velocity profiles of part a in Figures 18 through 23. The symmetry of the velocity proflles is somewhat distorted, Indicating the jets are disturbing the flowfield. The oscillations visible along the centerline of the test-section in Figure $4 \mathrm{c}$ are not related to shifts in the PVC axis. Part $c$ of Figures 18 through 23 show the swirl axis and hence, the PVC, to remain along the test-section centerline.

\section{CLOSURE}

\subsection{Conclusions}

Experlments have been conducted to obtain the time-mean and turbulent quantitles of opposed lateral jets in a low speed, nonreacting flowfield. A jet-to-crossflow velocity ratlo of $R$ $=4$ only, with crossflow inlet swirler vane angles $\phi=0$ (swirler removed), 45, and 70 degrees were investigated. Flow visualization techniques, with neutrally-buoyant helium-filled soap bubbles and multi-spark photography, were used to Identify flowfield characteristics such as jet penetration into the flowfleld. Time-mean quantities were determined using a six-orientation single hot-wire technique.

Flow visualization shows that the jets do not penetrate past the centerline of the test-section in the nonswirling case, $\phi=0$ (swirler removed). Measurements, using the six-orfentation single hotwire technique, indicated that this is indeed the case. In the swirling cases, the crossflow tends to remain in the region near the wall. The jets are deflected from their vertical course into a spiral course with the main flow. The jets were also found not to deflect the axis of the precessing vortex core. In the case of moderate swirl, swirl vane angle $\phi=45$ degrees, the jets could be tracked following a spiral trajectory. With strong swirl, swirl vane angle $\phi=70$ degrees, the jet trajectories are difficult to determine. The data indicate that symmetry of the velocity profiles is disturbed due to the presence of the jets for the $x / D$ locations considered. Jet penetration into the crossflow is reduced due to the additional velocity component introduced into the crossflow by swirl.

As previously mentioned, there are uncertainties concerning the accuracy of the calculated values of the turbulence quantities. The present data acquisition system is limited to a 

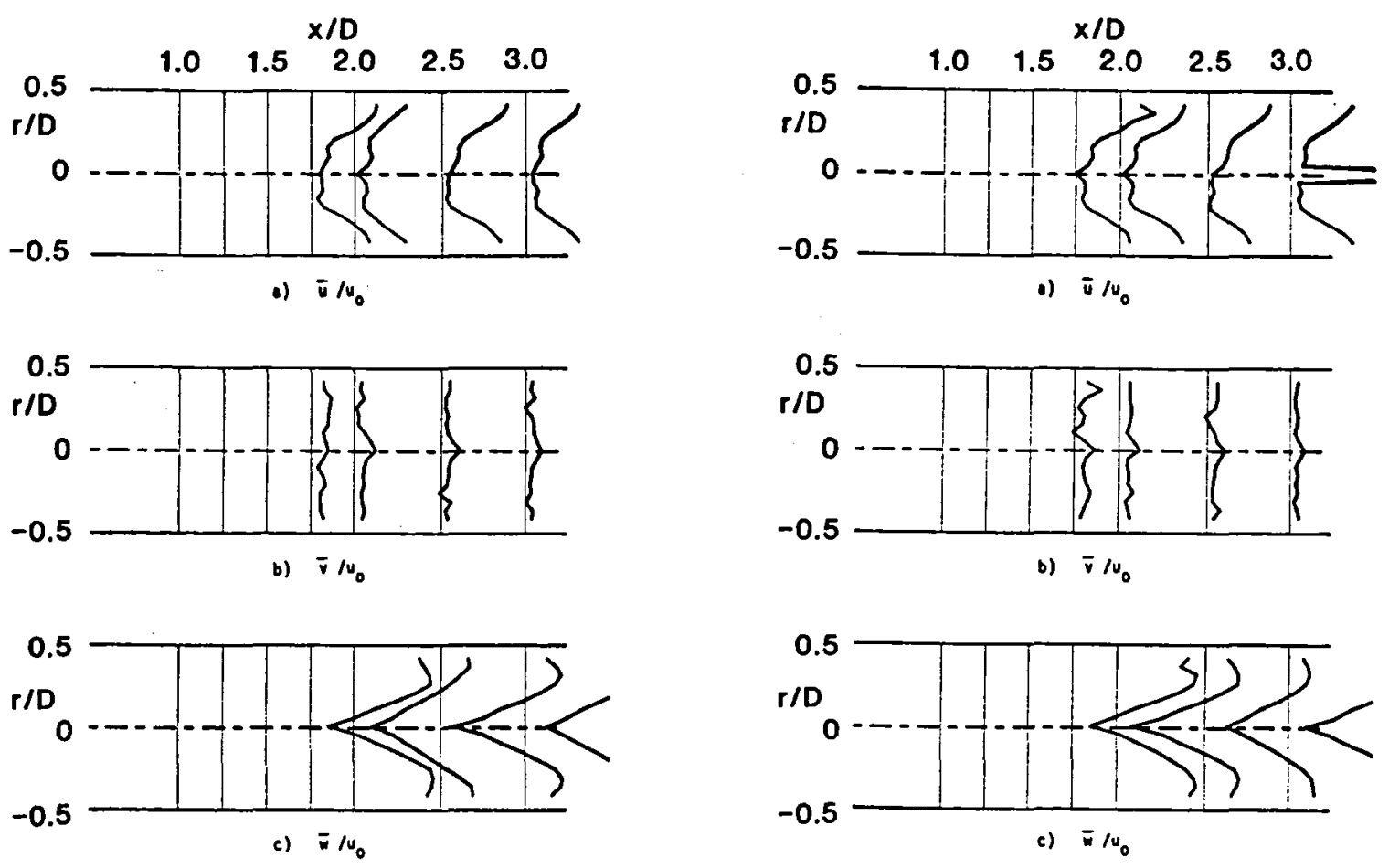

F1g. 18.T1me-Mean Velocity with $\phi=70$ Degrees, Traverse Angle $\theta=0$ Degrees.

Fig. 20.Time-Mean Velocity with $\phi=70$ Degrees, Traverse Angle $\theta=300$ Degrees.
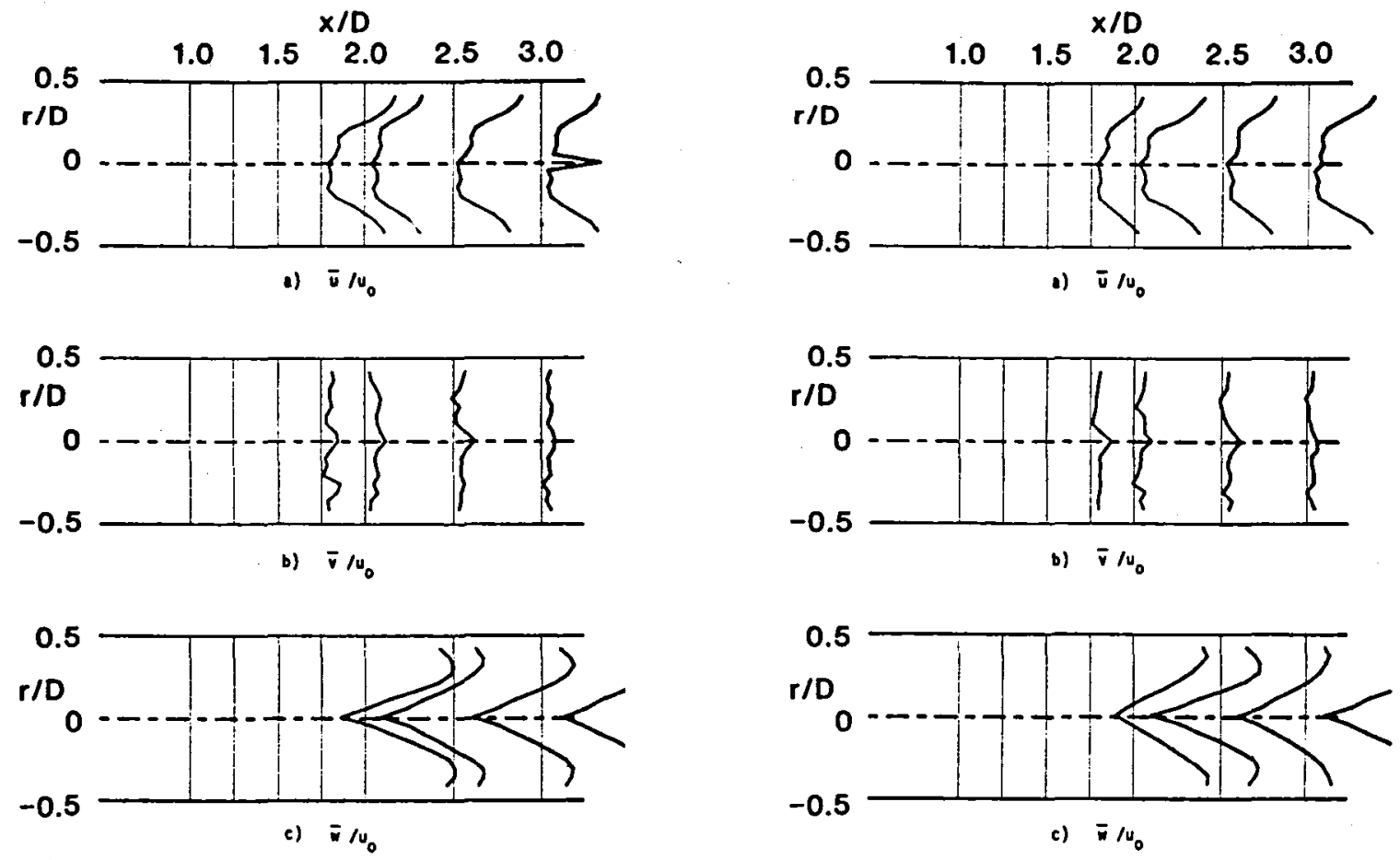

Fig. 19.Time-Mean Velocity with $\phi=70$ Degrees, Traverse Angle $\theta=330$ Degrees.

Fig. 21.Time-Mean Velocity with $\phi=70$ Degrees, Traverse Angle $\theta=270$ Degrees. 

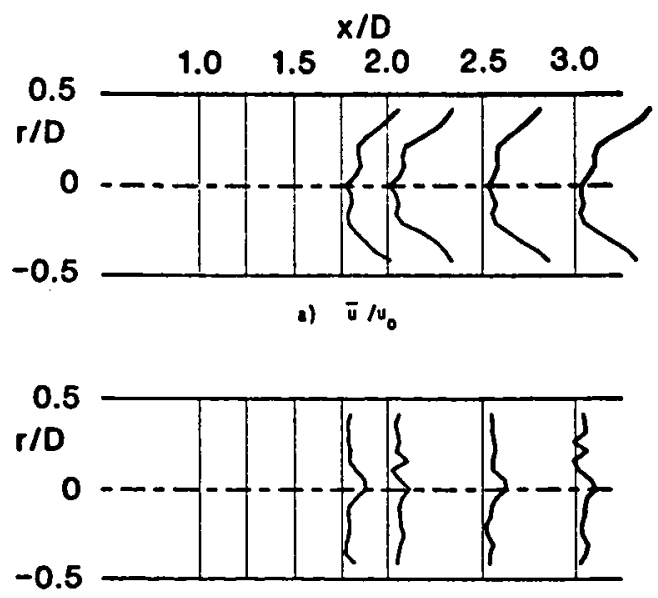

b) $\bar{v} / \omega_{0}$

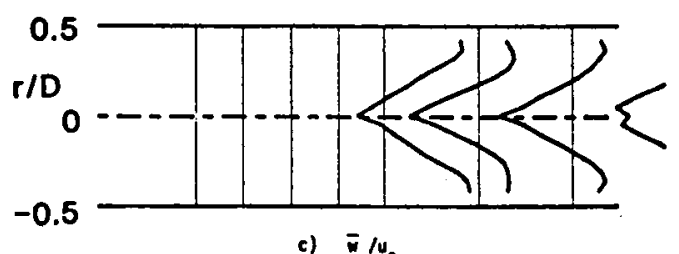

F18. 22. Time-Mean Velocity with $\phi=70$ Degrees, Traverse Angle $\theta=60$ Degrees.

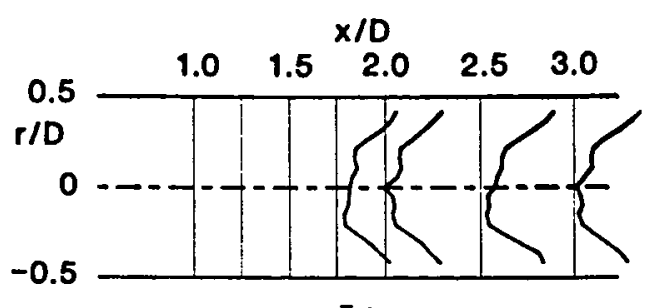

a) $\bar{u} / \omega_{0}$
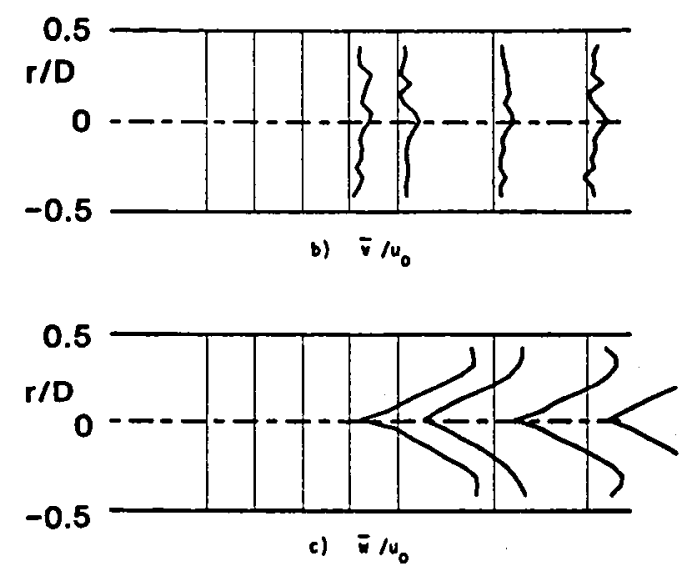

Fig. 23. Time-Mean Velocity with $\phi=70$ Degrees, Traverse Angle $\theta=30$ Degrees. sampling rate of $1000 \mathrm{~Hz}$. To decrease any allasing effects, higher turbulence frequencies should be flitered out. The accuracy of the turbulence quantity calcuations may also be increased by conducting an energy spectrum analysis to determine the relevant range of turbulence frequencles. Increasing the sampling rate to frequencles above the range of the relevant turbulent frequencles, combined with appropriate filtering, could greatly increase the accuracy of turbulent quantity measurements.

\subsection{Recommendations for Further Hork}

Th1s experiment is the first investigation into the effects of opposed jets on time-mean and turbulent quantities in swirling crossflow. Ong ${ }^{9}$ presents the case of one laterally injected jet, jet-to-crossflow velocity rat10 $R=4$, with swirl vane angle $\phi=0,45$, and 70 degrees. Further experiments should be carried out for jet-tocrossflow velocity ratios of $R=2,6$, and 8 with swirl vane angle $\phi=0,45$ and 70 degrees for each velocity ratio. Later experiments should include investigations into multiple jets located at the same axial location, as well as axially-staggered multiple jets.

\section{ACKNOWLEDGMENTS}

Appreciation is expressed to NASA Lewis Research Center, Grant No. NAG 3-549, technical monitor Dr. James D. Holdeman, for the financlal support of this project.

\section{REFERENCES}

1. Schetz, J. A., "Injection and Mixing in Turbulent Flow." Prog. in Astro. and Aero., AIAA, Vol. $68,1980$.

2. Lefebvre, A. H. "Gas Turbine Combustion." McGraw-H111, New York, 1983.

3. Gupta, A. K., Lilley, D. G., and Syred, N. "Swirl Flows." Abacus Press, Tunbridge Wells, England, 1984.

4. Gupta, A. K. and Lilley, D. G., "Flowfleld Modeling and Diagnostics." Abacus Press, Tunbridge Wells, England, 1985.

5. Ferrell, G. B., "Deflected Jet Experiments in a Turbulent Combustor Flowfield." Ph.D. Thesis, OKlahoma State University, St1llwater, Okla., Dec. 1984. See also: Ferrell, G. B. and Lilley, D. G. "Deflected Jet Experiments in a Turbulent

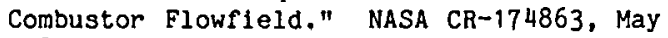
1985.

6. Ferrell, G. B., Abujelala, M. T., Busnalna, A. A., Lilley, D. G., "Lateral Jet Injection into Typical Combustor Flowflelds." AIAA Paper 84-0374, Reno, Nevada, Jan. 9-12, 1984.

7. Ferrell, G. B., Aoki, K. and Lilley, D. G. "Flow Visualization of Lateral Jet Injection into Swirling Crossflow." AIAA Paper 85-0059, Reno, Nevada, Jan. 14-17, 1985. 
8. Ferrell, G. B. and Lilley, D. G. "Turbulence Measurements of Lateral Jet Injection into Confined Tubular Crossflow." Paper AIAA85-1102, Monterey, Calif., July 8-10, 1985.

9. Ong, L. H., "Measurements of a Single Lateral Jet Injected into Swirling Crossflow." M.S. Thesis, Oklahoma State University, St1llwater, Okla., Dec. 1985.

10. Lilley, D. G., "Investigation of Flowfields Found in Typlcal Combustor Geometries." NASA CR-3869, Feb. 1985.

11. Lilley, D. G., "Swirling Flows in Typical Combustor Geometries." Paper AIAA-85-0184, Reno, Nevada, Jan. 14-17, 1985. See also: AIAA J. of Propulsion and Power, Vol. 2, 1986 (in press).

12. Chassaing, P., George, J., Claria, A., and Sananes, F., "Physical Characteristics of Subsonic Jets in a Cross-Stream." Journal of Fluid Mechanics, Vol. 62, 1974, p. 4164.

13. Rathgeber, D. E., and Becker, H. A., "Mixing Between a Round Jet and a Transverse Pipe Flow.". Proceedings of 1st Symposium on Turbulent Flows, Penn State University, 1977, pp. 10.17-10.26.

14. Crabb, D., Durao, D. F. G., and Whitelaw, J. W., "A Round Jet Normal to a Cross-Flow." ASME Trans., J, of Flulds Engr., Vol. 103, 1981, pp. 142.153.

15. Holdeman, J. D., Srinivasan, R., and Berenfeld, A., "Experiments in Dilution Jet Mixing." AIAA Journal, Vol. 22, No. 10, Oct. 1984, pp. 1436-1443.

16. Morel, T., "Comprehensive Design of Axisymmetric Wind Tunnel Contractions." ASME Paper 75-FE-17, Minneapolis, MN, May $5-7,1975$.

17. Merzkirch, W., "Flow Visualization." Academic Press, New York and London, 1974, pp. 191196.

18. Janjua, S. I., "Turbulence Measurements in a Complex Flowfleld Using a Six-Orientation Hot-Wire Probe Technique." M.S. Thesis, Oklahoma State University, Stillwater, Okla., Dec. 1981.

19. Jackson, T. W., "Turbulence Characteristics of Swirling Flowfields." Ph.D. Thes1s, OkIahoma State University, Stillwater, OK, Dec. 1983. See also: Jackson, T. W. and Lilley, D. G. "Turbulence Characteristics of Swirling Flowflelds." NASA CR-174918, May 1985.
20. Scharrer, G. L., "Swirl, Expansion Ratio and Blockage Effects on Continued Turbulent Flow." M. S. Thesis, Oklahoma State University, Stillwater, Okla. May 1984.

21. McMurry, C. B., "Experiments on Two Opposed Lateral Jets Injected into Swirling Crossflow." M.S, Thes1s, OkIahoma State University, Stillwater, Okla., December 1985. 
APPENDIX F

MULTISPARK FLOW VISUALIZATION OF LATERAL

JET INJECTION INTO A SWIRLING CROSS FLOW

JOURNAL OF PROPULSION AND POWER, VOL. 1 , NO. 6 , NOV. -DEC. 1985, pp. $485-487$ 


\title{
Multispark Flow Visualization of Lateral Jet Injection into a Swirling Cross Flow
}

\author{
G. B. Ferrell, ${ }^{*}$ K. Aoki, $†$ a d D. G. Lilleył \\ Oklahoma State University, Stillwater, Oklahoma
}

\begin{abstract}
Multispark flow visualization experiments have been conducted to characterize the time-mean flowfield of a deflected turbulent jet in a confining cylindrical cross flow. Jet/cross-flow velocity ratios of 2, 4, and 6 were investigated, under cross-flow inlet swirler vane angles of 0 (swirler removed), 45 , and 70 deg. The flow visualization results highlight interesting features of the flowfield, as well as the trajectory and spread pattern of the deflected jet.
\end{abstract}

\begin{tabular}{ll} 
& \multicolumn{1}{c}{ Nomenclature } \\
$D$ & $=$ test section diameter \\
$d_{j}$ & $=$ lateral jet diameter \\
$R$ & $=$ jet cross-flow velocity ratio, $v_{j} / u_{0}$ \\
$u, v, w$ & $=$ axial, radial, and swirl velocity components \\
$x, r, \theta$ & $=$ axial, radial, and azimuthal coordinates \\
$\phi$ & $=$ swirl vane angle \\
Subscripts & \\
0 & $=$ cross flow \\
$j$ & $=$ lateral jet
\end{tabular}

\section{Introduction}

$\mathbf{T}$ HE focus of the present study is to present the main characteristics of a deflected turbulent jet entering laterally into confined tubular nonexpanded cross flow that may also possess swirl. This situation occurs in gas turbine and ramjet combustors and the present ongoing experimental research effort is to provide a data base for turbulence model advances used by combustor designers. The specific objective of this investigation was to use a multispark flow visualization technique to highlight the important features of the flowfield. Gross flowfield characterization was obtained for a range of lateral jet/cross-flow velocity ratios $R=v_{j} / u_{0}=2,4$, and 6 and a range of inlet swirl strengths in the cross flow, with inlet swirl vane angles of $\phi=0$ (swirler removed), 45 , and $70 \mathrm{deg}$. The ratio of the cross flow to the lateral jet diameter is $D / d_{j}=10$. The experimental test facility, nozzle design, and construction details are given in recent documents. ${ }^{1-3}$

Earlier studies on the flowfield without lateral injection jets have been summarized. ${ }^{1}$ Preliminary observations and predictions for the lateral injected jet were given in Ref. 2. A

Received Dec. 14, 1984; presented as Paper 85-0059 at the AIAA 23rd Aerospace Sciences Meeting, Reno, NV, Jan. 14-17, 1985; revision received June 3,1985. This paper is declared a work of the U.S. Government and therefore is in the public domain.

*Graduate Student, School of Mechanical and Aerospace Engineering; presently Assistant Professor, Department of Mechanical Engineering, Texas Tech University, Lubbock. Member AIAA.

tVisiting Scholar, School of Mechanical and Aerospace Engineering, also Associate Professor, Department of Mechanical Engineering, Tokai University, Japan. Member AIAA.

$\ddagger$ Professor, School of Mechanical and Aerospace Engineering. Associate Fellow AIAA. recent report ${ }^{3}$ includes flow visualization, multiorientation single hot-wire measurements, and an extensive review of this phenomenon. Recent textbooks review and present the progress that has been made. Schet $z^{4}$ concentrates on the physics and modeling of injection and mixing of turbulent flows; Lefebvre ${ }^{5}$ acknowledges that the phenomena are of paramount importance in the combustion and dilution zones and presents recent progress and its relevance to combustor design requirements.

Many methods have been used in the manifestation of flowfields; the text by Merzkirch ${ }^{6}$ on flow visualization is particularly commendable. Smoke injection, helium bubble injection, and tuft grid methods have been used in earlier studies without any lateral jets. ${ }^{1}$ The present study employs a multispark visualization method, ${ }^{7}$ from which quantitative velocity deductions are also possible using the method described by Nakayama et al. ${ }^{8,9}$

\section{Multispark Flow Visualization}

Flow visualization is used for primary identification and characterization of the flowfield, with three techniques being used in the research program. ${ }^{2,3}$ Bubbles, because of their reflective qualities and neutral buoyancy in the airflow, provide an excellent medium to determine the paths of the flow trajectories. Smoke, because of its low comparative density and its tendency to mix well in the flow, makes an excellent medium to follow the flow and to accent the turbulent paths and recirculation zones in the flow. A more novel flow visualization technique employed in these investigations is the multispark method, which is the focus of the present paper.

The spark-gap equipment schematic is shown in Fig. 1. The pulse generating circuit and pulse transformer are manufactured by Sugawara Laboratories, Inc., Tokyo. The equipment is capable of producing pulse trains of up to 200 pulses at frequencies of $1-75 \mathrm{kHz}$. The output energy of the pulse is $0.05-0.5 \mathrm{~J}$ at voltages of $20-250 \mathrm{kV}$. As used in the present study, the electrodes are placed on opposite sides of the test section, typically one electrode above and one below the test section with a $15 \mathrm{~cm}$ spark gap. Approximately 40 sparks are used with $0.5 \mathrm{~J} / \mathrm{spark}$ at a voltage of $100 \mathrm{kV}$. Each spark pulse duration is approximately $1 \mu \mathrm{s}$; time between pulses is approximately $1 \mathrm{~ms}$.

When a high-voltage source is sparked across an air gap, an ionized path is created. Subsequent sparks will follow the current position of this low-resistance ionized path. By judicious placement of the electrodes in the wall boundary layer, where there is essentially zero velocity (next to wall), 
several discharges can follow the original ionized path as it moves with the fluid. It is necessary to have a lowconductivity test section material (for example, acrylic) so as not to interfere with spark paths. The spark itself provides sufficient lighting for the photographs. One camera (side view) is used for photographs with zero swirl. Two cameras (side and end view) are used simultaneously in the swirl cross-flow cases to give added perspective to the threedimensional features of the resulting flowfield.

\section{Results and Discussion}

Figures 2-4 present spark-gap flow visualization pictures for the cases of $R=2,4$, and 6 , using the method described in the previous section. Parts a-c in the figures correspond to $\phi=0$ (swirler removed), 45 , and $70 \mathrm{deg}$ of swirl in the cross flow, respectively. These particular photographs were taken with the electrodes positioned at $x / D=1.50$ where the jet enters at $x / D=1.00$.

In part a of these figures, the camera is positioned to the side of the facility and a vertical $r x$ plane is observed. In the swirl flow cases of parts $b$ and $c$, a second camera was simultaneously operated from a downstream location to illustrate the $r \theta$ plane behavior of the sparks. In these swirl cases, both photographs have been combined to form a common picture. The respective cases of $R=2,4$, and 6 with no swirl exhibit the change in the flowfield from $x / D=1.50$ and continuing downstream. The case of $R=2$ shows how the flowfield is merely deflected upward by the entering jet. The lower part of the arcs apparently are deflected around the jet, away from the previously vertical plane, and hence a true three-dimensional effect is perceived in the photograph. The case of $R=4$ shows the flowfield acceleration above and around the jet, which has its centerline nearly corresponding with the centerline of the tube. The "fold-over" just above the jet centerline probably corresponds to the downflow around the jet as the jet displaces the cross flow in the upper half of the test section. The case of $R=6$ shows less uniform behavior. The ares do appear to define the upper bounds of the jet and the turbulent region behind the jet, as observed in other flow visualization evidence using bubbles and smoke. ${ }^{2,3}$

The swirl flows presented in parts b and $c$ of Figs. 2-4 are actually two photographs taken simultaneously by separate cameras; the two negatives are combined to print a common picture. Again, the electrodes were placed at $x / D=1.50$, with the lateral jet entering at $x / D=1.00$. A wire was placed in the centerline of the tube to prevent the spark from arcing to the tube walls and to help define the tube centerline. The end views exhibit a great deal of reflection off of the inside acrylic tube walls: only the central portion of the view is useful. With moderate swirl $(\phi=45 \mathrm{deg})$, the injected jets have little effect on the main flow swirl patterns. However, the $R=6$ case is seen to deflect upward the central core of the swirl flow. The swirl strength seems to be depressed in the lower part of the test section by the additional momentum of the lateral jet. With strong swirl ( $\phi=70 \mathrm{deg})$, the cases of $R=2$ and 4 seem to slightly inhibit the swirl strength near the outer edge, whereas the $R=6$ case appears to exert its influence more inward in the central region of the flow: swirl

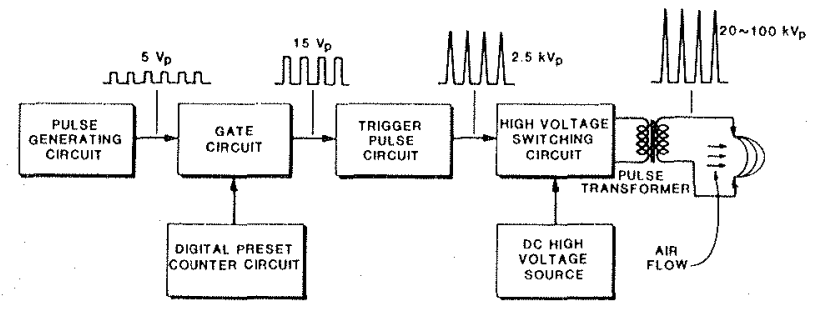

Fig. 1 Spark-gap equipment schematic. a) $\phi=0 \mathrm{deg}$.

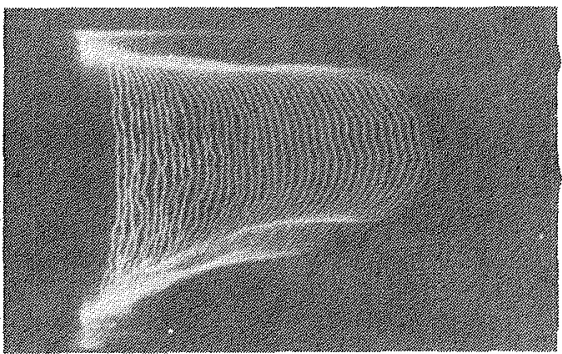

b) $\phi=45$ deg.

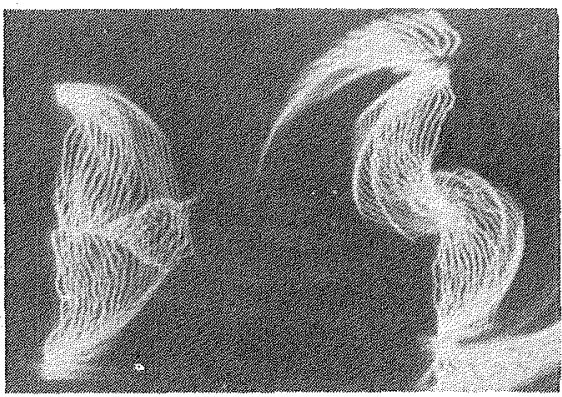

c) $\phi=70 \mathrm{deg}$.

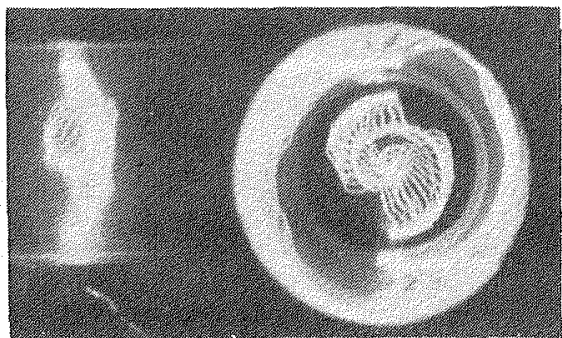

Fig. 2 Spark-gap flow visualization for jet/cross-flow velocity ratio $=\mathbf{2 . 0}$

a) $\phi=0$ deg.

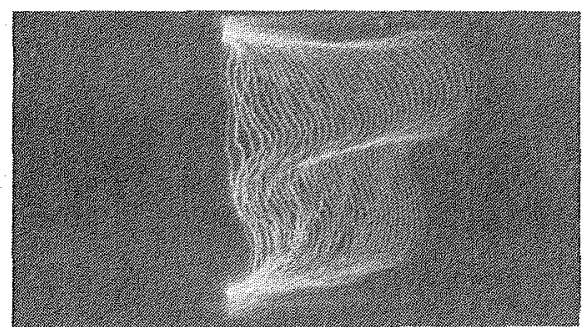

b) $\phi=45 \mathrm{deg}$

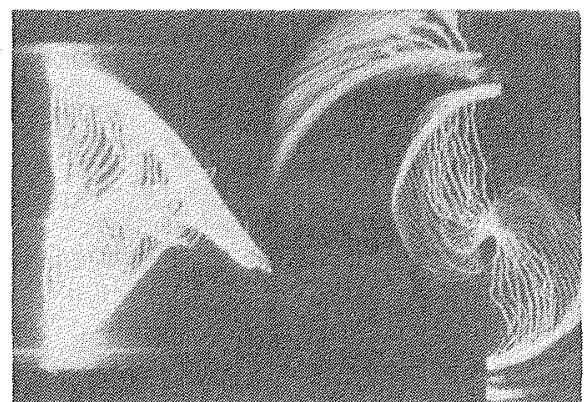

c) $\phi=70 \mathrm{deg}$

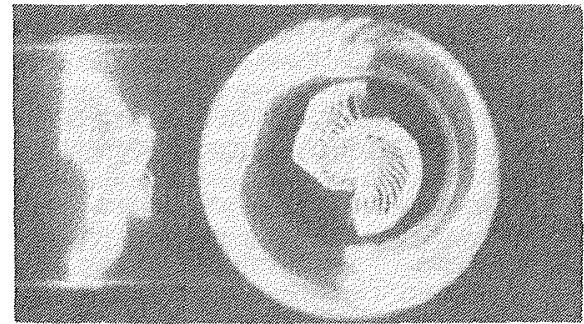

Fig. 3 Spark-gap flow visualization for jet/cross-flow velocity ratio $=4.0$ 
a) $\phi=0 \mathrm{deg}$.

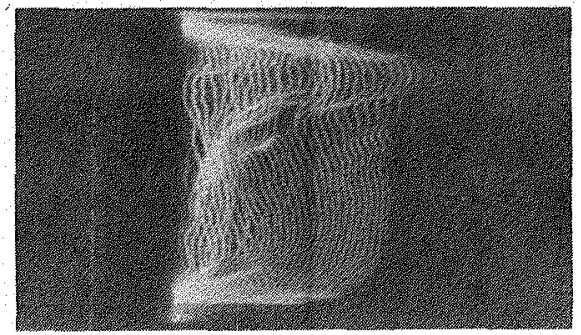

b) $\phi=45 \mathrm{deg}$.

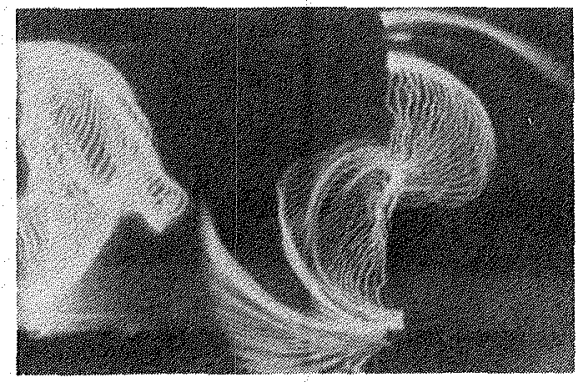

c) $\phi=70 \mathrm{deg}$.

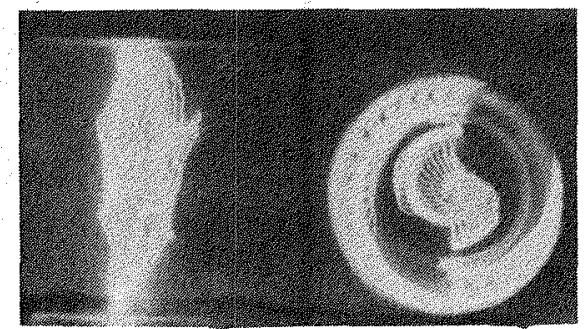

Fig. 4 Spark-gap flow visualization for jet/cross-flow, velocity ratio $=6.0$

levels in the lower central region are reduced and the central core of the main swirl flow is deflected upward. These results all confirm in general other flow visualization evidence, ${ }^{3}$ which leads to a reduced need for detailed experimental measurements.

\section{Conclusion}

The multispark flow visualization technique was used to define changes in the flowfield after injection of a lateral jet.
The technique demonstrated the bulk effects of range of jet velocity ratios on the main flow, with a range of inlet swirl strengths in the main flow. Multispark photographs taken in the cases with swirl demonstrate the effect of lateral jet momentum of the main flow, including: elevation of the vortex core location, reduction of swirl strength in the lower part of the test section, and extent of mixing and spreading of the jet. In conjunction with other flow visualization evidence, which defines better the lateral jet trajectories, a reduced need for detailed experimental measurements results.

\section{Acknowledgments}

Gratitude is expressed to NASA Lewis Research Center for assistance via Grant NAG 3-549, technical monitor Dr. J. D. Holdeman. Help with the the experiments came from several students, notably Bobby W. Evans, Steve S. Meador, Lawrence H. Ong, and Mark A. Southard, to whom special thanks are offered.

\section{References}

${ }^{1}$ Lilley, D. G., "Swirling Flows in Typical Combustor Geometries," NASA CR-3869, Feb. 1985.

${ }^{2}$ Ferrell. G. B., Abujelala, M. T., Busnaina, A. A., and Lilley, D. G., "Lateral Jet Injection into Typical Combustor Flowfields," AIAA Paper 84-0374, Jan. 1984.

${ }^{3}$ Ferrell, G. B. and Lilley, D. G., "Deflected Jet Experiments in a Turbulent Combustor Flowfield," NASA CR-174863, Feb. 1985.

${ }^{4}$ Schetz, J. A., AIAA Progress in Aeronautics and Aeronautics: Injection and Mixing in Turbulent Flow, Vol, 68, AIAA, New York, 1980.

${ }^{5}$ Lefebvre, A. H., Gas Turbine Combustion, McGraw-Hill Book Co., New York, 1983.

${ }^{6}$ Merzkirch, W., Flow Visualization, Academic Press, New York/London, 1974, pp. 191-6.

${ }^{7}$ Meador, S. S. and Southard, M. A., "Multi-Spark Visualization of Typical Combustor Flowfields," Senior Project Report, School of Mechanical and Aerospace Engineering, Oklahoma State University, Stillwater, Dec. 1984.

${ }^{8}$ Nakayama, Y., Okitsu, S, Aoki, K., and Ohta, H., "Flow Direction Detectable Spark Method," The International Symposium on Flow Visualization, Tokyo, Oct. 1977, pp. 163-168.

${ }^{9}$ Nakayama, Y., Aoki, K., and Ohta, H., and Wakatsuki, M., "Spark Method Visualization of the Swirling Flow Mixing Process," Proceedings of the 3rd Method, International Symposium on Flow Visualization, Ann Arbor, MI, Sept. 1983, pp. 481-485. 


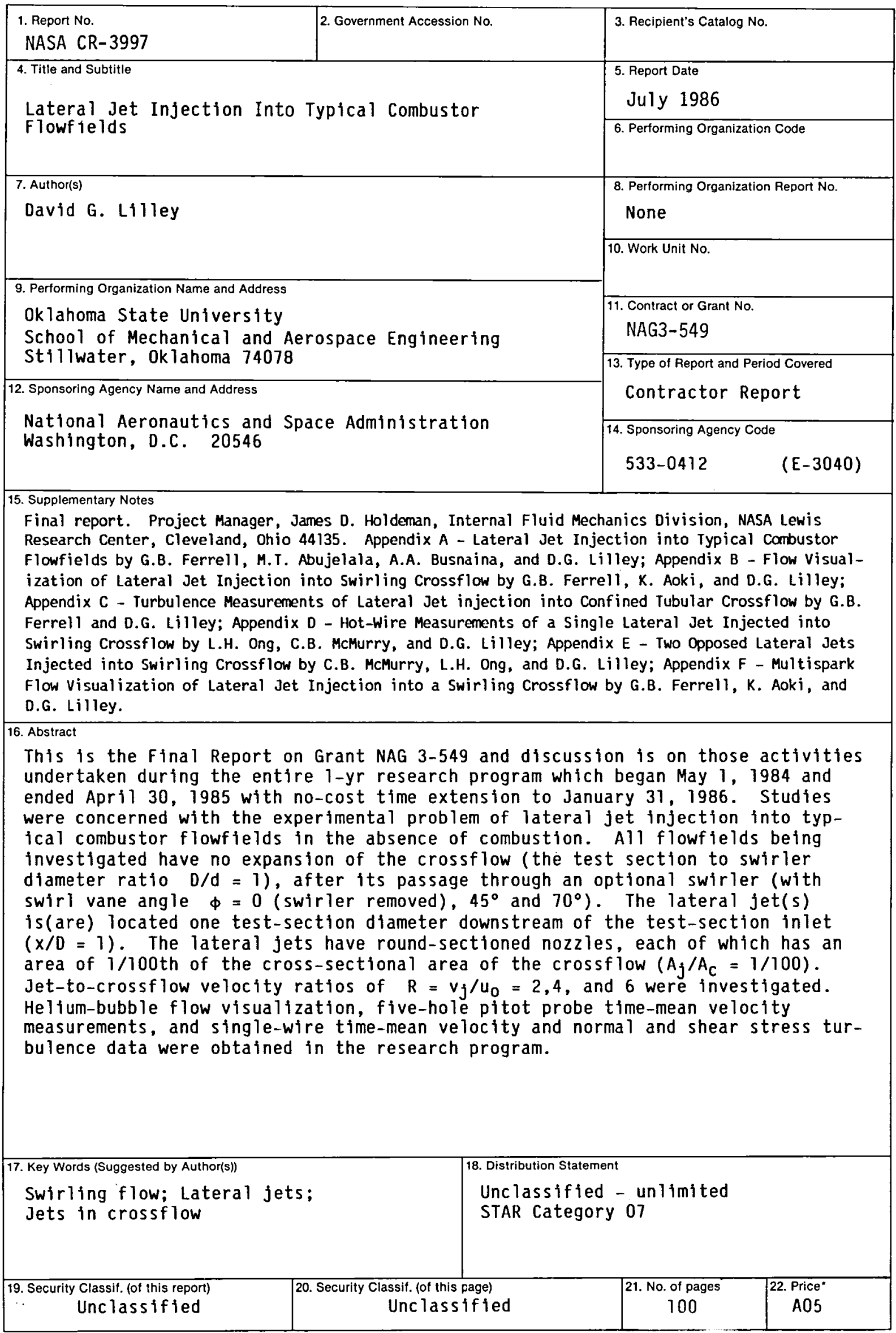




\section{End of Document}

